\title{
AN ANNOTATED AND ILLUSTRATED CATALOGUE OF POLYPORES (AGARICOMYCETES) OF THE BIALOWIEŻA FOREST (NE POLAND)
}

\author{
DARIUSZ KARASIŃSKI ${ }^{1} \&$ MAREK WOŁKOWYCKI
}

\begin{abstract}
The Białowieża Forest (BF) is one of the best-preserved lowland deciduous and mixed forest complexes in Europe, rich in diverse fungi. This paper summarizes what is known about the poroid fungi of the Polish part of the Białowieża Forest, based on literature data, a re-examination of herbarium materials, and the authors' studies from 1990-2014. An annotated catalogue of polypores recorded in the forest is presented, including 80 genera with 210 species. All literature and herbarium records are enumerated, and 160 species are illustrated with color pictures. Fourteen species previously reported in the literature have uncertain status because they lack voucher specimens and were not confirmed in recent field studies. Antrodiella subradula (Pilát) Niemelä \& Miettinen, previously known from Asia, is reported for the first time from Europe. Fourteen species are newly reported from the Białowieża Forest (mainly from Białowieża National Park), including 8 species with first records in Poland (Antrodia hyalina Spirin, Miettinen \& Kotir., Antrodia infirma Renvall \& Niemelä, Antrodiella subradula, Junghuhnia fimbriatella (Peck) Ryvarden, Postia folliculocystidiata (Kotl. \& Vampola) Niemelä \& Vampola, Postia minusculoides (Pilát ex Pilát) Boulet, Skeletocutis chrysella Niemelä, Skeletocutis papyracea A. David), and 6 species reported previously from other localities in Poland [Antrodiella faginea Vampola \& Pouzar, Dichomitus campestris (Quél.) Domański \& Orlicz, Loweomyces fractipes (Berk. \& M. A. Curtis) Jülich, Oxyporus latemarginatus (Durieu \& Mont.) Donk, Perenniporia narymica (Pilát) Pouzar, Phellinus nigricans (Fr.) P. Karst.]. Several very rare European polypores already reported from the Białowieża Forest in the $20^{\text {th }}$ century, such as Antrodia albobrunnea (Romell) Ryvarden, Antrodiella foliaceodentata (Nikol.) Gilb. \& Ryvarden, Buglossoporus pulvinus (Pers.) Donk, Dichomitus albidofuscus (Domański) Domański and Gelatoporia subvermispora (Pilát) Niemelä, were found at new localities, confirming their continuous occurrence in this forest.
\end{abstract}

Key words: Basidiomycota, Biosphere Reserve, fungal diversity, Poland, poroid fungi, primeval forests, UNESCO World Heritage Site

Dariusz Karasiński, Department of Mycology, W. Szafer Institute of Botany, Polish Academy of Sciences, Lubicz 46, $31-512$ Kraków, Poland; e-mail: d.karasinski@botany.pl

Marek Wołkowycki, Białystok University of Technology, Faculty of Forestry in Hajnówka, Pitsudskiego 8, 17-200 Hajnówka, Poland

\section{INTRODUCTION}

The Białowieża Forest $(\mathrm{BF})$ is one of the best preserved lowland deciduous and mixed forest complexes in Europe (Faliński 1986; Peterken 1996; Jędrzejewska \& Jędrzejewski 1998). It is reported as an example of a European non-fragmented virgin forest community (e.g., Parviainen 2005) or as a remnant of culturally modified ancient forest (e.g., Bobiec 2012). The whole forest complex covers an area of $1250 \mathrm{~km}^{2}$ and is located at the border between Poland and Belarus. The Polish part lies between $52^{\circ} 39^{\prime}-52^{\circ} 48^{\prime} \mathrm{N}$ and $23^{\circ} 34^{\prime}-23^{\circ} 38^{\prime} \mathrm{E}$

\footnotetext{
1 Corresponding author
}

and covers an area of $646 \mathrm{~km}^{2}$, including $105 \mathrm{~km}^{2}$ protected since 1921 as the Białowieża National Park (BNP) and $c a 120 \mathrm{~km}^{2}$ protected as a forest reserve (Faliński 2002; Okołów 2012). Białowieża National Park was declared a Biosphere Reserve in 1977 and in 1979 was designated a UNESCO World Heritage Site (Okołów 2002, 2009). Since 2005 the Biosphere Reserve has been expanded to cover the whole Polish part of the Białowieża Forest (Okołów 2012). Glaciofluvial sands, gravels and clays built the flat, undulating plain on which the forest is situated at $135-190 \mathrm{~m}$ a.s.l. (Kwiatkowski 1994). The local climate has both continental and 
Atlantic features (Faliński 1986). During the last 50 years the mean annual temperature was $6.9^{\circ} \mathrm{C}$ (January mean $-3^{\circ} \mathrm{C}$, July mean $18.3^{\circ} \mathrm{C}$ ), snow cover lasted 92 days on average, and mean annual precipitation was $627 \mathrm{~mm}$ (Malzahn et al. 2009). The Białowieża Forest consists of a mosaic of various forest communities determined by the variation of topography, soil and hydrology. Coniferous and mixed coniferous stands dominated by Pinus sylvestris L. and Picea abies (L.) H. Karst. cover $\mathrm{ca} 50 \%$ of the forest area in the Polish part of the Białowieża Forest. Wet deciduous forest with Alnus glutinosa Gaertn. and Fraxinus excelsior L. covers $c$ a $20 \%$, rich mesic deciduous stands with Quercus robur L., Carpinus betulus L., Tilia cordata Mill. and Acer platanoides L. cover 15\%, and early successional stands with Betula pendula Roth and Populus tremula L. cover $13 \%$ of the forest area (Jędrzejewska \& Jędrzejewski 1998). The Białowieża Forest differs from Western European forests in the absence of Fagus sylvatica L. The abundance of Quercus robur differentiates it from Eastern European forests. Picea abies occurs in almost every forest community (Pawlaczyk 2009).

The first published data on the polypores of the Białowieża Forest date to the $19^{\text {th }}$ century (Błoński et al. 1888). Over the last 127 years about 100 publications have included information on these fungi, implying that the diversity of the polypores of that area is relatively well known, but poroid species new to science are still being described from the Białowieża Forest (Niemelä et al. 2012; Miettinen et al. 2012). Our knowledge of polypore diversity has not been deliberately summarized for a long time, although some information was included in publications from Stanisław Domański (e.g., Domański 1965, 1967, 1972b; Domański et al. 1967, 1973). Up to 2013 these publications were the primary source of knowledge on polypore diversity in the Białowieża Forest. Some records of a number of rare poroid species given by Stanisław Domański have not been confirmed by any subsequent researchers. Recently some new records of selected poroid species (but mostly common ones) were published based on material collected for fungal exhibits organized yearly in September by Białowieża National Park (e.g., Szczepkowski et al. 2010, 2011; Gierczyk et al.
2013, 2014). Niemelä (2013) published a manual for identification of polypores of the Białowieża Forest, including descriptions and a list of species found by his team during inventory work in 2008-2012. This manual gives information on 177 poroid species reported from the study area - 142 species found during the inventory and 35 species from reports in the literature. The literature data are given without revision of the herbarium materials, which in some cases results in duplication of unverified and sometimes wrong information mainly from Domański (records based on misidentified specimens; some of them corrected here).

This work summarizes what is known about the poroid fungi of the Polish part of the Białowieża Forest in the form of an annotated and illustrated catalogue. It is based on literature data, a re-examination of some herbarium materials, especially for species whose concept has changed, and our studies from 1990-2014.

\section{MATERIAL AND METHODS}

The original as-yet unpublished material was collected in different areas of the Białowieża Forest by the first author in 2005-2014 during a few short collecting trips in 2005-2008, 2011, 2013 and 2014, and extensive inventory work in 2009-2010 for the "Conservation plan for species of macrofungi in the Białowieża National Park' (Karasiński et al. 2010). In total, more than 1100 specimens of polypores were collected and studied. The material is preserved mainly in the personal reference collection of the first author (abbreviated D.K.), with duplicates in KRAM F. The second author has collected polypores in the Białowieża Forest since 1990. The collection includes $\mathrm{ca} 350$ specimens stored in the Herbarium of Marek Wołkowycki (abbreviated H.M.W. M). Other specimens examined ( $c a$ 150) were obtained from KRA and mostly from KRAM F-SD (collection of Stanisław Domański in KRAM F, containing mainly polypores collected in the Białowieża Forest in 1955-1970).

For micromorphological studies, thin freehand sections were cut with a razor blade from fresh or dry basidiomata under a Nikon SMZ-2T microscope, mounted in water, $3 \%$ aqueous potassium hydroxide with $1 \%$ aqueous phloxine, and Melzer's reagent or $0.1 \%$ cotton blue in $60 \%$ lactic acid (Kirk et al. 2008), and examined under a Nikon Eclipse E-400 microscope at magnification up to $1250 \times$. Color photographs were 


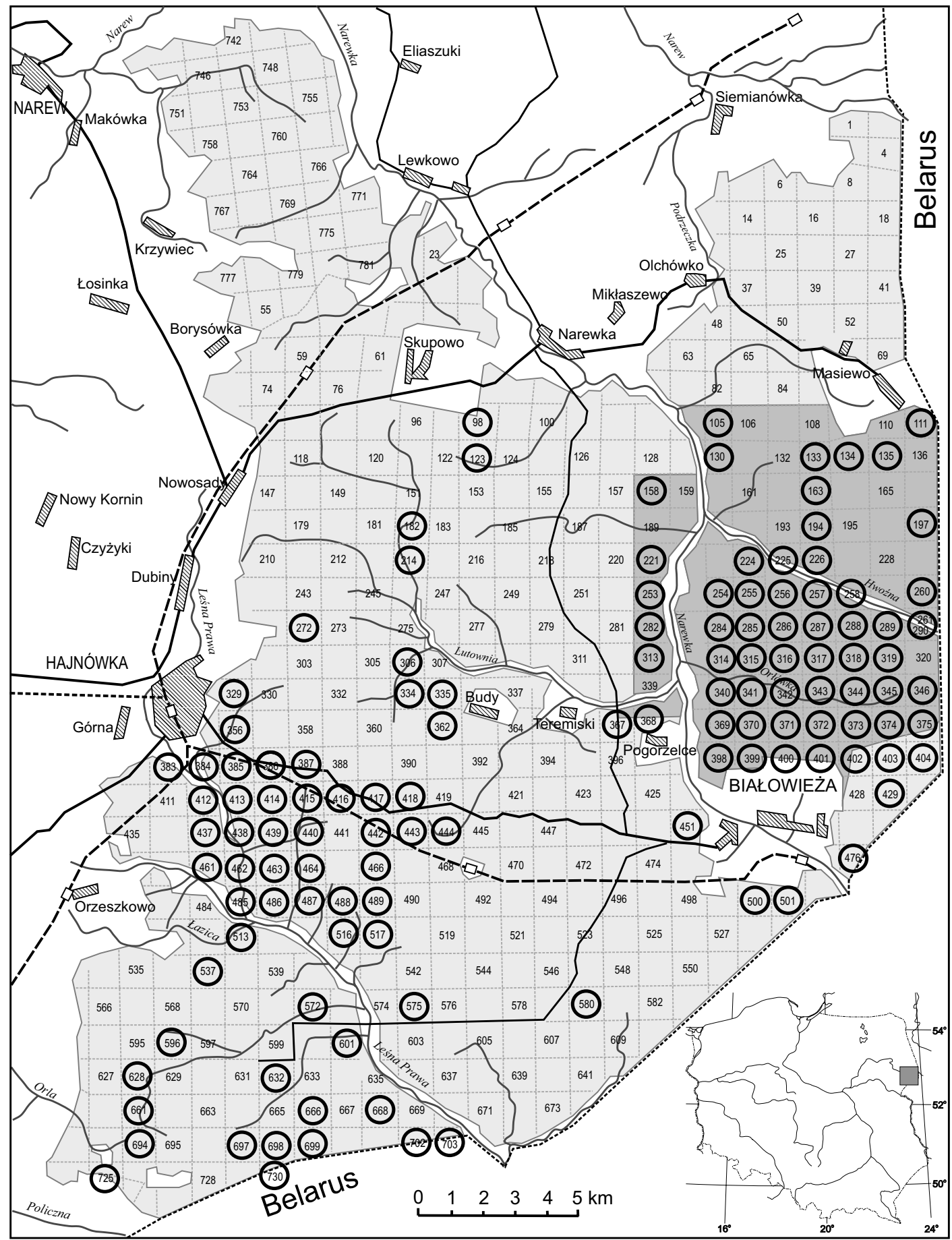

Fig. 1. Study area: pale grey - Białowieża Forest, dark grey - Białowieża National Park, black circles - visited localities. Numbers on map refer to forest section numbers. From Karasiński et al. 2009 (supplemented and modified). 
taken by the first author using a Nikon D200 camera and an AF Micro Nikkor $60 \mathrm{~mm} \mathrm{1:2.8} \mathrm{D} \mathrm{lens.} \mathrm{My-}$ coBank (www.mycobank.org) and Index Fungorum (www.indexfungorum.asp) websites were consulted for nomenclature and abbreviations of authors of the fungal names.

In the catalogue the species appear alphabetically. All specimens are cited for most species. For some frequently collected species only selected representative specimens examined are cited in full, and for the remaining specimens only the forest section numbers and associated specimen/herbarium numbers are given. For all specimens cited in full the data are arranged as follows: number of forest section of the Polish part of Białowieża Forest in bold (often with precision to section quarter), substrate, date of collection, collector name, specimen number, and herbarium abbreviation. The arrangement of the forest sections in the Białowieża Forest, and the visited collecting sites, are shown in Figure 1. For localities outside the borders of the Białowieża National Park, information about the precise site is usually given in parentheses after the forest section numbers. For some herbarium specimens deposited in the collection of Stanisław Domański, some original annotations, especially original determinations from the labels, are cited after the KRAM F-SD numbers. The literature reports of each species are given as reference lists arranged chronologically. Dubious reports are excluded from the main list and included in the section 'Records with uncertain status', with appropriate commentary.

As a rule, the collecting sites of the specimens collected by Domański in the Białowieża Forest are marked as collected in 'Białowieża'. Likewise, for almost all his literature records, precise site locations besides 'Białowieża' are not available. For such specimens cited here, only 'Białowieża Forest' is given as the locality instead of forest section numbers.

\section{RESULTS AND DISCUSSION}

\section{LIST OF SPECIES}

Abbreviations used in the list: Alnus - Alnus glutinosa; Betula - Betula pendula; $\mathrm{BF}$ - Białowieża Forest; BNP Białowieża National Park; Carpinus - Carpinus betulus;
Fraxinus - Fraxinus excelsior; Picea - Picea abies; Pinus - Pinus sylvestris; Populus - Populus tremula; Quercus - Quercus robur

Abortiporus biennis (Bull. ex Fr.) Singer Fig. 2A

LITERATURE REPORTS: Jaroszewicz 1996, Niemelä 2013.

REMARKs. The specimen reported by Jaroszewicz (1996) was presented during the Second Fungi Exposition of the Białowieża Forest. Unfortunately, herbarium material was not preserved, but the occurrence of the species in the BF was recently confirmed by Niemelä (2013).

\section{Albatrellus ovinus (Schaeff.) Murrill Fig. 2B}

SPeCimens EXAmined: 134D, on the ground among mosses under Picea, 15 Sept. 2005, Karasiński 0509157831 (D.K.); 632A (Topiło), on the ground under Picea, Carpinus, Quercus and Betula, 28 Sept. 2006, Karasiński 060928-6235 (D.K.).

Literature RePORTS: Orłoś 1955b (as Polyporus ovinus), Orłoś 1960 (as Caloporus ovinus), Orłoś 1961 (as Caloporus ovinus), Domański et al. 1967, Bujakiewicz et al. 1992, Skirgiełło 1997, Szczepkowski et al. 2008, Gierczyk et al. 2013, Niemelä 2013.

\section{Amylocystis lapponica (Romell) Bondartsev}

\& Singer ex Singer Fig. 2C

Specimens eXamined: 260D, on Picea fallen trunk, 12 Oct. 2009, Karasiński 4633a (D.K.); 257D (D.K. 4347); 284 (D.K. 6342); 285A, on Picea fallen trunk, 29 July 2009, Karasiński 3617c (D.K.); 287A (D.K. 4368); 288D (D.K. 3738, 3743D); 289C (D.K. 3745); 314B (D.K. 3888A); 315A (D.K. 3896B); 315B (D.K. 3911); 319C (D.K. 3733A); 319C (D.K. 3579C); 319C (D.K. 3748A); 319C (D.K. 3766A); 340C (D.K. 4225C); 340F (D.K. 4243A); 340G (D.K. 4316); 343B (D.K. 3841E); 344A (D.K. 3836A); 345B (D.K. 3718C); 345B (D.K. 3716C); 346A (D.K. 3728B, 4679D); 370B (D.K. 4756); 370C (D.K. 3703); 372B (D.K. 3984B); 374A (D.K. 4162); 374B (D.K. 4170A); 374C (D.K. 3409); 374D (D.K. 3176A); 375A (D.K. 3577); 375B (D.K. 4279A); 399A (D.K. 5063C); 399B (D.K. 5072A).

Fig. 2. A - Abortiporus biennis (Bull. ex Fr.) Singer (specimen photographed in the Kampinos National Park, central Poland), B - Albatrellus ovinus (Schaeff.) Murrill, C - Amylocystis lapponica (Romell) Bondartsev \& Singer ex Singer, D - Amyloporia crassa (P. Karst.) Bondartsev \& Singer ex Bondartsev, E - Amyloporia sinuosa (Fr.) Rajchenb., Gorjón \& Pildain, F - Amyloporia sitchensis (D. V. Baxter) Vampola \& Pouzar, G - Amyloporia xantha (Fr.) Bondartsev \& Singer ex Bondartsev, H - Anomoporia bombycina (Fr.) Pouzar. Photo D. Karasiński (A-H). 

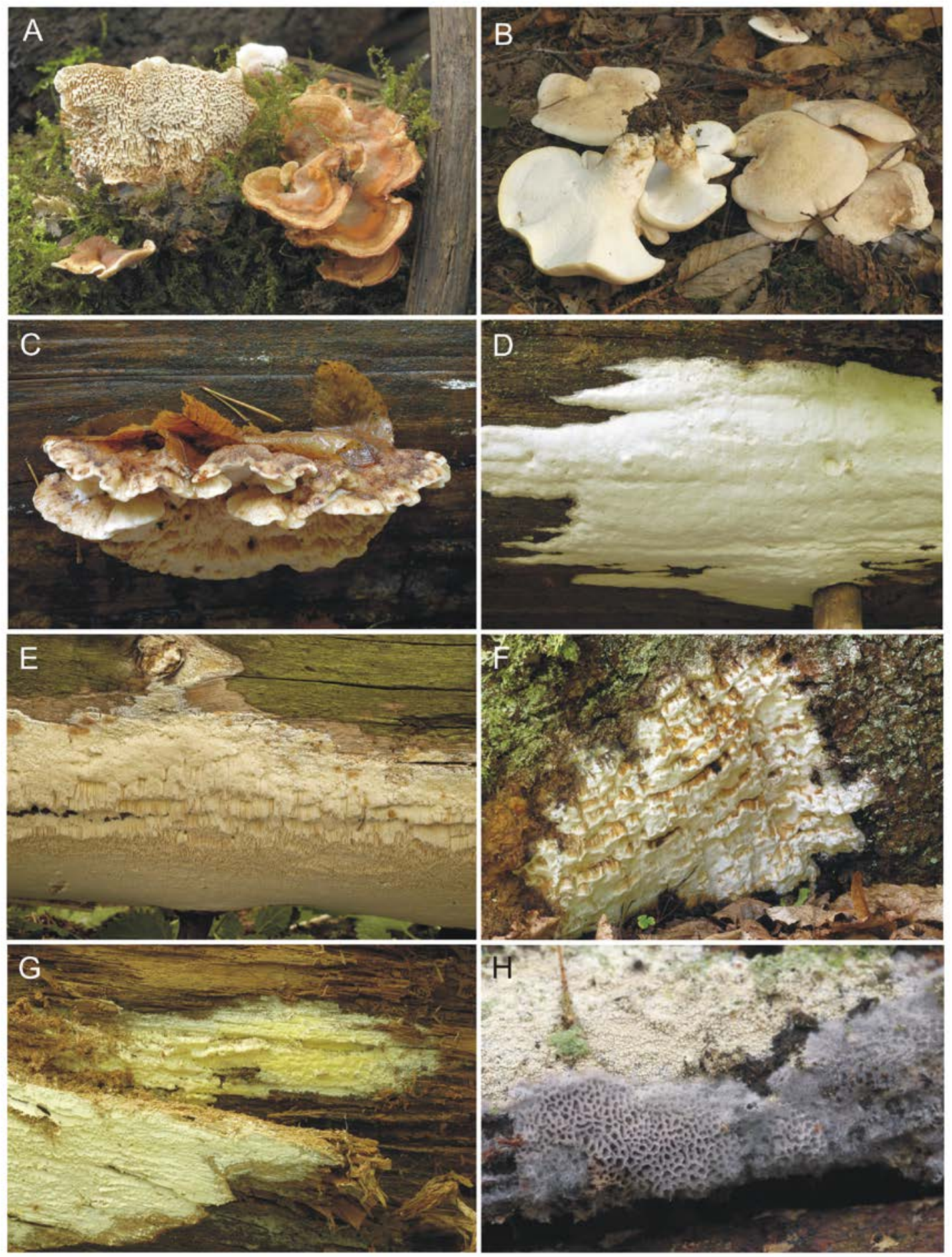
Literature RePORTS: Domański 1959b (as Leptoporus lapponicus), Gumińska 1963 (as Leptoporus lapponicus), Domański et al. 1967, Domański 1967, Kotlaba \& Lazebniček 1967, Kreisel 1967, Anonymous 1968, Domański 1972a, Domański et al. 1973, Piątek 2005a, Szczepkowski et al. 2008, Gierczyk et al. 2013, Niemelä 2013.

REMARKS. All specimens reported here were collected from logs, trunks and snags of Picea. Amylocystis lapponica is rather common in BNP, especially in the former Orłówka Strictly Protected Area, and very rare in other parts of the BF. In Poland it has never been found outside the BF (Piątek 2005a). The specimen cited by Wojewoda (2003 after Domański 2001) from the Lasy Łochowskie forest (central Poland) represents Postia fragilis (Fr.) Jülich (A. Szczepkowski, pers. comm.).

Amyloporia crassa (P. Karst.) Bondartsev \& Singer ex Bondartsev

Fig. 2D

SPecimens examined: 256D, on Picea log, 21 Sept. 2009, Karasiński 4328 (D.K.); 316D, on Picea log, 1 May 2010, Karasiński 5361 (D.K.); 316, on Picea fallen trunk, 14 Aug. 2009, Karasiński 3784A (D.K.); 317C, on Picea fallen trunk, 14 Aug. 2009, Karasiński $3786 B$ (D.K.); 319C, on Picea fallen trunk, 28 July 2009, Karasiński 3602 (D.K.); 319C, on Picea fallen trunk, 13 Aug. 2012, Karasiński 3756 (D.K.); 345A, on Picea fallen trunk, 11 Aug. 2009, Karasiński 3716 (D.K.); 346, on Picea fallen trunk, 11 Aug. 2009, Karasiński 3724 (D.K.); 374, on Picea fallen trunk, 15 Sept. 2009, Karasiński 4150 (D.K.); 374, on Picea fallen trunk, 15 Sept. 2009, Karasiński 4177 (D.K.); 375, on Picea fallen log, 19 Sept. 2009, Karasiński 4287 (D.K.).

Literature Reports: Domański 1959a (as Poria crassa), Domański 1965 (as Amyloporia crassa f. crassa), Domański 1967, Anonymous 1968, Domański 1972b, Niemelä 2013 (as Antrodia crassa).

Remarks. Domański (1965) described Amyloporia crassa f. subimbricata using specimens collected in the BF. Some specimens of this taxon (but not all) preserved in Domański's herbarium (e.g., KRAM F-SD 333, 792, 2658, 2859, 2862, 2863, 2864) represent $A$. sitchensis (D. V. Baxter) Vampola \& Pouzar. See also remarks under Amyloporia sitchensis.
Amyloporia sinuosa (Fr.) Rajchenb., Gorjón \& Pildain

Fig. 2E

Specimens eXAmined: 130, on Picea fallen trunk, 14 Sept. 2009, Karasiński 4113 (D.K.); 135C, on Picea fallen log, 8 July 2009, Karasiński 3342c (D.K.); 214B (D.K. 11007); 224, on Picea fallen trunk, 19 Aug. 2009, Karasiński 3947 (D.K.); 225D (D.K. 4598); 260D, on Picea trunk, 12 Oct. 2009, Karasiński 4633 b (D.K.); 261A (D.K. 3380); 282 (D.K. 3522); 285 (D.K. 5299E); 288D (D.K. 3737B); 314B (D.K. 3303); 316 (D.K. 5273-1); 319C (D.K. 3754E); 341 (D.K. 4771); 344A (D.K. 3837); 346 (D.K. 4688); 368 (D.K. 4084); 369 (D.K. 3487); 386 (D.K. 4084); 399, on Picea fallen trunk, 2 Nov. 2009, Karasiński 5062 (D.K.); 400 (D.K. 3258); 402B (H.M.W. M-3646); 439A (Głęboki Kąt Reserve), on Picea fallen trunk, 22 May 2006, Karasiński 060522-5410 (D.K.); 485C (H.M.W. M-1307); 699C (H.M.W. M-3600).

Literature RePORTs: Pilát 1950 (as Poria sinuosa), Domański 1965 (as Coriolellus vaporarius f. vaporarius and C. vaporarius f. bulbosus), Domański 1967 (as Coriolellus sinuosus), Kotlaba \& Lazebniček 1967 (as Coriolellus sinuosus), Anonymous 1968 (as Coriolellus vaporarius), Domański 1972b (as Coriolellus sinuosus), Szczepkowski et al. 2008 (as Antrodia sinuosa), Niemelä 2013 (as Antrodia sinuosa), Gierczyk et al. 2014 (as Antrodia sinuosa).

REMARKS. This species is very common in the study area.

Amyloporia sitchensis (D. V. Baxter) Vampola \& Pouzar

Fig. 2F

Specimens eXamined: 225B, on Picea fallen log, 14 Sept. 2009, Karasiński 4146 (D.K.); 226A, on Picea fallen trunk, 30 July 200, Karasiński 3683 (D.K.); 253C, on Picea fallen log, 13 Sept. 2009, Karasiński 4092 (D.K.); 255D, on Picea fallen log, 26 Apr. 2010, Karasiński 5243 (D.K.); 285A, on Picea fallen trunk, 28 Apr. 2010, Karasiński 5288 (D.K.); 286D, on Picea fallen trunk, 14 Aug. 2009, Karasiński 3774 (D.K.); 288D, on Picea fallen trunk, 12 Aug. 2009, Karasiński 3743 (D.K.); 289C, on Picea fallen log, 12 Aug. 2009, Karasiński 3746 (D.K.); 314B, on Picea fallen log, 15 Aug. 2009, Karasiński 3800 (D.K.); 314C, on Picea fallen log, 25 Apr. 2010, Karasiński $5180 A$ (D.K.); 315A, on Picea fallen trunk, 18 Aug. 2009, Karasiński 3890 (D.K.); 316, on bark of Picea stump, 14 Aug. 2009, Karasiński 3797 (D.K.); 318D, on Picea fallen trunk, 17 Aug. 2009, Karasiński 3871 (D.K.); 319C, on Picea stump, 13 Aug. 2009, Karasiński 3754d (D.K.); 340C, 
on Picea stump, 6 July 2009, Karasiński 3288 (D.K.); 341D, on Picea fallen trunk, 17 Oct. 2009, Karasiński 4776 (D.K.); 342D, on Picea fallen log, 1 May 2010, Karasiński 5345 (D.K.); 343B, on Picea fallen log, 16 Aug. 2009, Karasiński 3844 (D.K.); 344B, on Pinus fallen log, 16 Aug. 2009, Karasiński 3833 (D.K.); 370D, on Picea fallen log, 5 July 2009, Karasiński 3271 (D.K.); 372B, on Populus fallen trunk, 20 Aug. 2009, Karasiński 3980 (D.K.); 373C, on Picea fallen log, 13 July 2009, Karasiński 3471 (D.K.); 374C, on Picea fallen log, 11 July 2009, Karasiński 3395 (D.K.); 375A, on Pinus fallen log, 27 July 2009, Karasiński 3564 (D.K.); 399, on Picea fallen trunk, 1 Nov. 2009, Karasiński 5011A (D.K.); 439A (Głęboki Kąt Reserve), on Picea fallen trunk, 22 May 2006, Karasiński 060522-5371 (D.K.).

Literature RePORTS: Domański 1965 (as Amyloporia crassa f. subimbricata), Domański 1972b (as Amyloporia crassa f. subimbricata), Vampola \& Pouzar 1992, Szczepkowski et al. 2008 (as Antrodia sitchensis), Szczepkowski et al. 2010 (as Antrodia sitchensis), Gierczyk et al. 2013 (as Antrodia sitchensis), Niemelä 2013 (as Antrodia sitchensis), Gierczyk et al. 2014 (as Antrodia sitchensis).

REMARKs. In the BF it grows almost exclusively on Picea, but was once collected from Pinus and once from Populus. In Poland Amyloporia sitchensis is very rare and occurs in natural forests of the northeastern part of the country. So far, outside the BF it was only recorded in the Starożyn Reserve in the Puszcza Augustowska forest (Vampola \& Pouzar 1992). The most beautiful perennial basidiomata with an undulate hymenial surface form on the bark in root axils on rootnecks of Picea stumps and dead standing trees. In the field it can be differentiated from similar $A$. crassa by its sweet resinous odor, which is less pronounced but preserved also in herbarium specimens. Micromorphologically, the size and shape of basidiospores ( $c a 4.5-5.5 \times 1.8-2.2 \mu \mathrm{m}$, cylindrical) differentiate $A$. sitchensis from $A$. crassa (basidiospores 5-7 $\times$ 2.5-3.5 $\mu \mathrm{m}$, ellipsoidal). Moreover, the skeletal hyphae of $A$. sitchensis do not dissolve in $5 \%$ potassium hydroxide solution, while those of $A$. crassa dissolve rapidly in this medium. Amyloporia sitchensis sporulates in summer; samples collected in spring and autumn are often sterile. Vampola and Pouzar (1992) suggested that this fungus may be an undescribed Eu- ropean taxon morphologically very similar to the species described from North America and known as Antrodia (Amyloporia) sitchensis.

Amyloporia xantha (Fr.) Bondartsev \& Singer ex Bondartsev

Fig. 2G

SPeCimens EXAMINED: 135C, on Picea fallen log, 8 July 2009, Karasiński 3342a (D.K.); 224B, on Picea fallen trunk, 19 Aug. 2009, Karasiński 3939b (D.K.); 254D, on Picea fallen trunk, 12 Sept. 2011, Karasiński 6213 (D.K.); 256, on Pinus fallen trunk, 29 July 2009, Karasiński 3623 (D.K.); 260A, on Pinus fallen trunk, 12 Oct. 2009, Karasiński 4645 (D.K.); 285A, on Pinus fallen log, 28 Apr. 2010, Karasiński 5306 (D.K.); 318D, on Pinus fallen log, 17 Aug. 2009, Karasiński 3865 (D.K.); 374A, on Pinus fallen log, 26 July 2009, Karasiński 3540 (D.K.); 400B, on Picea fallen trunk, 5 July 2009, Karasiński 3260 (D.K.).

Literature RePORTs: Pilát 1950 (as Poria calcea var. xantha), Domański 1965 (as Amyloporia xantha f. xantha and A. xantha f. pachymeres), Domański 1967, Kotlaba \& Lazebniček 1967, Kreisel 1967, Anonymous 1968, Domański 1972b, Szczepkowski et al. 2008 (as Antrodia xantha), Niemelä 2013 (as Antrodia xantha), Gierczyk et al. 2014 (as Antrodia xantha).

REMARKS. This species is very common in the study area.

Anomoporia bombycina (Fr.) Pouzar Fig. 2H

SPECIMENS EXAMINED: 130A, on very rotten wood of Picea fallen log, 14 Sept. 2009, Karasiński 4116 (D.K.); 374C, on Pinus fallen log, 15 Sept. 2009, Karasiński 4157 (D.K.); 375D, on Picea fallen log, 16 Oct. 2014, Karasiński 10948 (D.K.); 398G, on very decayed wood of Picea fallen trunk, 24 July 2009, Karasiński 3503 (D.K.).

LiteratURE REPORTS: Domański 1965 (as Fibuloporia bombycina), Domański 1967 (as Fibuloporia bombycina), Kotlaba \& Lazebniček 1967, Anonymous 1968 (as Fibuloporia bombycina), Domański 1972b, Niemelä 2013.

Remarks. Anomoporia bombycina is associated with fallen logs and trunks of conifers (Picea, Pinus), usually in the late stage of wood decomposition. The species is very rare in Poland, known only from the BF. Only three specimens are deposited in Domański's herbarium (KRAM F-SD 719 and 740 as Poria bombycina, KRAM F-SD 741 as Fibuloporia bombycina) collected in 
1955-1958. Possibly the historical Polish record from the vicinity of Elbląg published by Kaufmann (1925) is based on misidentification, because the specimen was collected from Populus sp. Revision of that report is not possible due to lack of herbarium material, but it is likely that Kaufmann's specimen represents Ceriporiopsis aneirina (Sommerf.) Domański.

Antrodia albobrunnea (Romell) Ryvarden

Fig. 3A

Specimens eXamined: 256B, on well rotted Pinus fallen trunk, 29 July 2009, Karasiński 3624 (D.K.); 256, on Pinus fallen trunk, 19 Sept. 2011, Karasiński 6356 (D.K.); 256, on Pinus wood (fallen trunk fragment), 19 Sept. 2011, Karasiński 6366 (D.K.); 374C, on Pinus fallen log, 23 Sept. 2010, Karasiński 6009 (D.K.); 374C, on Pinus fallen log, 21 Sept. 2012, Karasiński 6405 (D.K.).

LITERATURE REPORTS: Domański 1965 (as Tyromyces albo-brunneus), Domański 1967 (as Tyromyces albobrunneus) Domański 1972b (as Tyromyces albobrunneus), Piątek 2005b, Niemelä 2013.

REMARKS. The single previous specimen of Antrodia albobrunnea collected in the BF is preserved in Domański's herbarium (KRAM F-SD 3733) and all literature reports referred to this specimen. Herbarium envelope KRAM F-SD 3733 contains a very small basidiome, ca $3 \times$ $1 \mathrm{~cm}$. Microstructures are well preserved and developed in this specimen, except for brown pigmented hyphae, which finally formed a brown marginal zone and a low subicular layer close to the substrate. They are present in small numbers in Domański's specimen, so the brown marginal zone is almost absent, hardly visible to the naked eye and also under a lens. The specimens collected in 2009-2012 and reported here are larger and better developed (especially Karasiński 6356 is a large basidiome). In Poland $A$. albobrunnea seems to be a true rarity, known only from the BNP (Piątek $2005 \mathrm{~b}$ ), and also here it is very rare.
Antrodia hyalina Spirin, Miettinen \& Kotir.

Fig. 3B

Specimens examined: 443A (W. Szafer Landscape Reserve), on Populus fallen trunk, 22 June 2008, Karasiński 1685 (KRAM F-47456); the same locality and host, 21 Apr. 2009, Karasiński 2839 (D.K.); 417D (W. Szafer Landscape Reserve), on Populus fallen trunk, 10 Oct. 2009, Karasiński 4566 (D.K.).

LiteratURE REPORTS: None. The species is new for Poland.

REMARKS. This recently described species (Spirin et al. 2013a) is similar to Antrodia pulvinascens (Pilát) Niemelä. Both species grow on Populus wood but $A$. hyalina differs by having annual basidiomata (perennial in A. pulvinascens), cylindrical basidiospores (ellipsoidal in $A$. pulvinascens) and solid skeletal hyphae (hyphae have a clearly visible lumen in $A$. pulvinascens).

\section{Antrodia infirma Renvall \& Niemelä}

Fig. 3C

Specimens eXAmined: 256D, on Pinus fallen trunk, 29 July 2009, Karasiński 3654 (D.K.); 319D, on Pinus log, 11 Aug. 2009, Karasiński 3717 (D.K.); 374D, on Pinus big log hanging 1-2 m above ground level, 26 July 2009, Karasiński 3553 (D.K.); the same locality and host, 11 Aug. 2009, Karasiński 3713 A (D.K.); 375B, on heartwood of Pinus fallen log, 27 July 2009, Karasiński 3585 (D.K.); 375D, on heartwood of Pinus fallen log, 27 July 2009, Karasiński 3565 (D.K.).

LITERATURE REPORTS: None. The species is new for Poland.

Remarks. Antrodia infirma was described from the boreal zone at the timberline in Lapland (Renvall \& Niemelä 1992). This species is characterized by having resupinate basidiomata with relatively large pores, a dimitic hyphal system with very rare tramal skeletals, and cylindrical basidiospores, $6.2-7.8 \times 2.2-3 \mu \mathrm{m}$ in the specimens studied. The characters of the Polish specimens very well fit the original diagnosis (Renvall \& Niemelä 1992) and the Swedish collection (Renvall 1993), except for basidiomata size. According to the

Fig. 3. A - Antrodia albobrunnea (Romell) Ryvarden, B - Antrodia hyalina Spirin, Miettinen \& Kotir., C - Antrodia infirma Renvall \& Niemelä, D - Antrodia malicola (Berk. \& M. A. Curtis) Donk, E - Antrodia pulvinasces (Pilát) Niemelä, F-Antrodia serialis (Fr.) Donk, G - Antrodiella citrinella Niemelä \& Ryvarden, H - Antrodiella foliaceodentata (Nikol.) Gilb. \& Ryvarden. Photo D. Karasiński (A-H). 

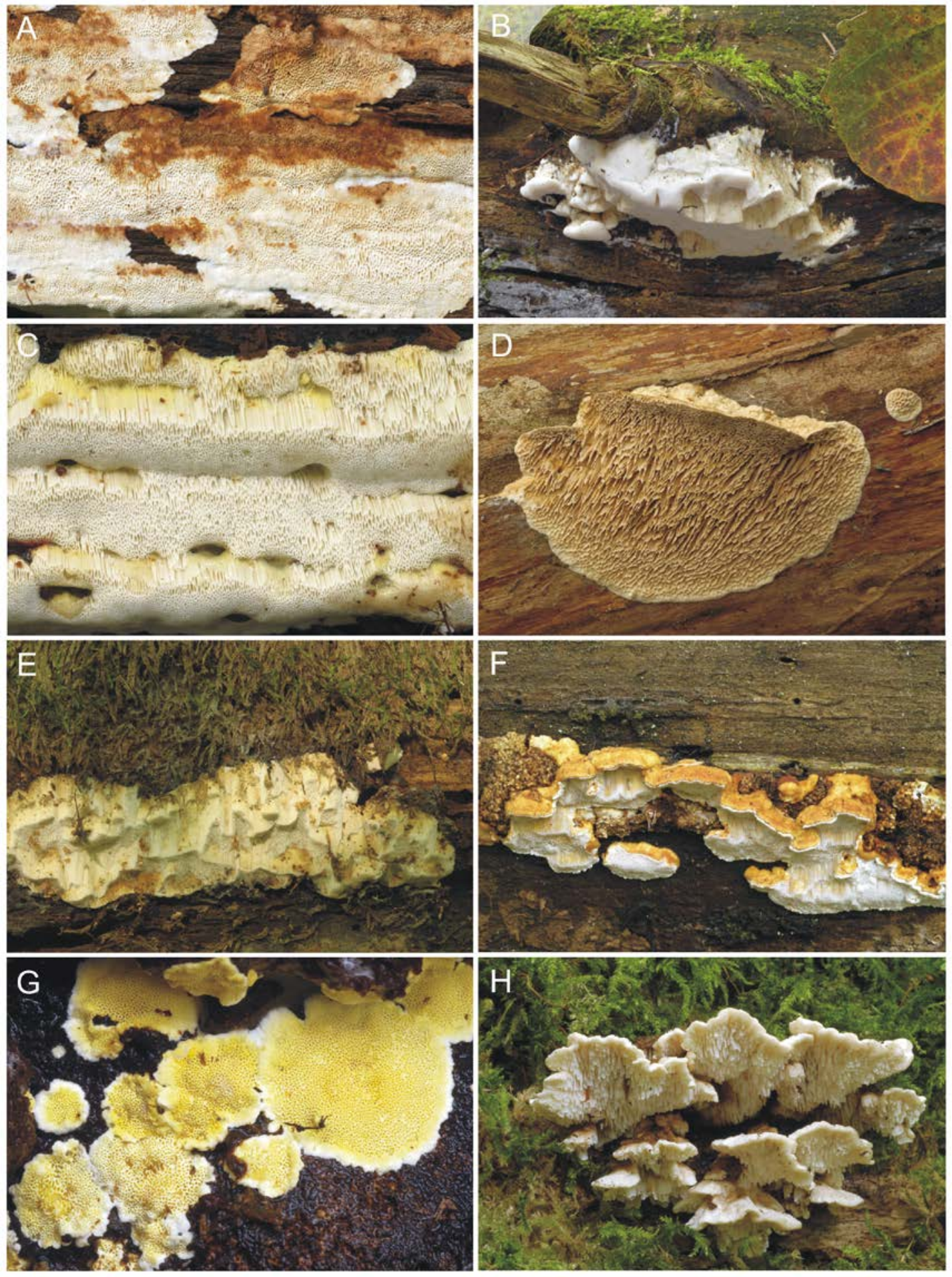
Scandinavian authors cited above and Ryvarden and Melo (2014), in the boreal zone this species forms small basidiomata up to $10 \mathrm{~cm}$ long, while the Polish specimens are medium-sized, large or very large, up to $50 \mathrm{~cm}$ long or even more when confluent. Antrodia infirma inhabits fallen trunks of conifers. About $92 \%$ of the Finish collections are from Pinus (Renval 1993); it was found only twice on Picea, in Finland and in Sweden. All the Polish samples are from large decorticate Pinus trunks in various stages of decomposition, from early stage shortly after decorticating to a fairly advanced stage of decay. In the latter case the basidiome was developed on heartwood of a big decayed pine log. The basidiomata of the species are short-lived and rapidly destroyed by insects. Old basidiomata look somewhat like white forms of Rhodonia placenta (Fr.) Niemelä, K.-H. Larss. \& Schigel. Gelatoporia subvermispora (Pilát) Niemelä forms slightly similar basidiomata.

Antrodia infirma is a rare European taxon known from boreal primeval forests. The Polish records from the BNP are at the southern distribution limit of the species. Antrodia infirma is known from Finland, Sweden, Russia (Renvall \& Niemelä 1992; Renvall 1993; Ryvarden \& Melo 2014) and northeastern Poland (this paper).

\section{Antrodia macra (Sommerf.) Niemelä}

Specimen eXAmined. BF, on Salix sp. branch, 23 Sept. 1965, Domański (KRAM F-SD 4884, labelled as 'Coriolellus heteromorphus (Fr.) Bond. form. albidus [Coriolellus serpens (Fr.) Bond.]').

\section{LITERATURE REPORTS: Niemelä 2013.}

REMARKS. The species appears to be very rare in the study area. It differs from $A$. serpens (Fr.) P. Karst. by having smaller pores and shorter basidiospores, and its association with Salix spp. Ryvarden and Gilbertson (1993) reported this species from Poland without a precise locality.

Antrodia malicola (Berk. \& M. A. Curtis) Donk

Fig. 3D

Specimen examined. 399C, on Populus dead standing trunk, 2 Nov. 2009, Karasiński 5046B (D.K.).

LITERATURE REPORTS: Skirgiełło 1998, Piątek 2002a.
REMARKS. It appears to be very rare in the study area and also in Poland, where it is known from less than 10 localities in the southeastern part of the country (Wojewoda 2003; Piątek 2002a).

Antrodia pulvinascens (Pilát) Niemelä Fig. 3E

SPeCimens EXAMINED: 260D, on Populus large fallen branch, 12 Oct. 2009, Karasiński 4629 (D.K.); 399B, on rotten Populus log, 1 Nov. 2009, Karasiński 5041 (D.K.); BNP, Sierchanowski Tryb, Populus tremula, very big fallen trunk in moist mixed forest, 14 Sept. 2009, Niemelä 8650 \& Schigel (KRA).

\section{LiTERATURE REPORTS: Niemelä 2013.}

Remarks. In Poland Antrodia pulvinascens is known so far only from the Tatra Mts (Niemelä 1978b as A. plicata) and the BF (Niemelä 2013). This species has perennial basidiomata and macromorphologically is very similar to Amyloporia sitchensis, but differs in the shape and size of the basidiospores, and the lack of the specific odor characterizing the latter. Antrodia hyalina, which also grows on Populus wood, differs in having annual basidiomata with a soft consistency and narrower, cylindrical basidiospores (ellipsoidal and significantly wider in $A$. pulvinascens).

Antrodia serialis (Fr.) Donk

Fig. 3F

Specimens eXAmined: 272 (Lipiny Reserve), on Picea fallen trunk, 21 May 2006, Karasiński 0605215267 (D.K.); 319C, on Picea fallen trunk, 13 Aug. 2009, Karasiński 3764 (D.K.); 369A, on Picea fallen trunk, 14 July 2009, Karasiński 3488A (D.K.); 370C, on Picea fallen trunk, 10 Aug. 2009, Karasiński 3702 (D.K.); 374, on Picea trunk, 23 Apr. 2009, Karasiński 2875 (D.K.); 375D (D.K. 10953); 412B (H.M.W. M-1835); 413A (H.M.W. M-1895); 414A (H.M.W. M-1948); 414B (H.M.W. M-1941); 415C (H.M.W. M-1475); 439A (H.M.W. M-1257); 439C (H.M.W. M-1296); 462A (H.M.W. M-1332); 485C (H.M.W. M-1312); 486B (H.M.W. M-1875); 572 (Michnówka Reserve), on Picea fallen log, 24 May 2006, Karasiński 0605245513 (D.K.).

Literature RePORTS: Błoński et al. 1888 (as Polyporus callosus), Pilát 1950 (as Trametes serialis), Orłoś 1955b (as Trametes serialis), Orłoś 1960 (as Trametes serialis), Orłoś 1961 (as Trametes serialis), Domański 1965 (as Coriolellus serialis and C. serialis f. callosus), Domański et al. 1967 (as Coriolellus serialis), Domański 
1967 (as Coriolellus serialis), Anonymous 1968 (as Coriolellus serialis), Domański 1972b (as Coriolellus serialis f. callosus), Szczepkowski et al. 2008, Gierczyk et al. 2013, Niemelä 2013, Gierczyk et al. 2014.

REMARKS. It is one of the commonest poroid species in the study area, growing on trunks and stumps of conifers, especially Picea. It forms annual to biennial, often large, resupinate or effusedreflexed basidiomata with a leathery consistency, white pores, and elongate and narrow pilei with an ochraceous to brown upper surface. The resupinate basidiomata of Antrodia serialis are slightly similar to A. albobrunnea, but the latter has a softer consistency, never forms pilei and produces allantoid basidiospores (subfusiform in A. serials).

\section{Antrodia serpens (Fr.) P. Karst.}

Specimens eXAmined: BF, on Corylus avellana dead trunk, 24 Oct. 1959, Domański (KRAM F-SD 487, labelled '[Trametes albida (Fr.) B. et G.] Coriolellus heteromorphus (Fr.) Bond. form. albidus').

Literature RePORTS: Domański 1965 (as Coriolellus albidus), Domański 1967 (as Coriolellus albidus), Domański 1972b (as Coriolellus albidus), Niemelä 2013 (as Antrodia heteromorpha).

REMARKS. The specimen examined is the same as listed in the literature reports as Coriolellus albidus. During contemporary fieldwork the occurrence of this species was not confirmed. It may be very rare or, less likely, its population is locally extinct. In Poland this species is quite common in deciduous and mixed forests (especially in beech forests on fallen branches of Fagus sylvatica) in the south of the country. Recently the species was epitypified based on material from Poland (Spirin et al. 2013b).

\section{Antrodiella citrinella Niemelä \& Ryvarden}

Fig. 3G

SPECIMENS EXAMINED: 226A, on Picea fallen trunk and Fomitopsis pinicola dead basidiome, 20 Sept. 2011, Karasiński 6389 (D.K.); 285A, on Betula pendula fallen trunk close to Fomitopsis pinicola dead basidiomata, 28 Apr. 2010, Karasiński 5312 (D.K.); 342C, on Picea fallen trunk and Fomitopsis pinicola dead basidiome, 17 Oct. 2009, Karasiński 4784 (D.K.); 370C, on Picea fallen trunk close to Fomitopsis pinicola dead basid- iome, 15 Oct. 2009, Karasiński 4731 (D.K.); 370, on Picea fallen trunk and Fomitopsis pinicola dead basidiome, 15 Oct. 2009, Karasiński 4747 (D.K.); 373C, on rotten Picea abies stump and Fomitopsis pinicola dead basidiome, 24 Apr. 2009, Karasiński 2896 (D.K.); 373D, on Picea fallen trunk close to Fomitopsis pinicola dead basidiome, 31 Oct. 2009, Karasiński 4988 (D.K.); 399A, on Picea fallen trunk and Fomitopsis pinicola dead basidiome, 1 Nov. 2009, Karasiński 5039 (D.K.); 399B, on Picea fallen trunk, 2 Nov. 2009, Karasiński 5058 (D.K.); 399D, on Picea fallen log, 2 Nov. 2009, Karasiński 5049 (D.K.); BF, on Picea, 25 Oct. 1956, Domański (KRAMF-SD 5369 as Tyromyces semisupinus).

LiterATURE REPORTS: Karasiński et al. 2009, Niemelä 2013, Gierczyk et al. 2014 (as Flaviporus citrinellus).

REMARKS. This species is uncommon in the BF. Resupinate or rarely effused-reflexed basidiomata with a vivid yellow hymenial surface, small, subglobose basidiospores, and connection with Fomitopsis pinicola are diagnostic characters of Antrodiella citrinella.

\section{Antrodiella faginea Vampola \& Pouzar}

SPeCimen EXAmined. 130, on Alnus fallen trunk with Mensularia radiata, 14 Sept. 2009, Karasiński 4126 (D.K.); 134A, on Alnus fallen log decayed by Mensularia radiata, 12 July 2009, Karasiński 3446 (D.K.); 368, on Quercus fallen branch with Hymenochaete rubiginosa, 13 Sept. 2009, Karasiński 4083 (D.K.); 374C, on Carpinus fallen branch, 11 July 2009, Karasiński 3421 (D.K.); 374C, on Betula fallen branch, 26 July 2009, Karasiński 3537 (D.K.); BF, on Populus, 14 Sept. 1967, Domański (KRAM F-SD 5303 as Tyromyces semisupinus); BF, on Carpinus, 9 Aug. 1965, Domański (KRAM F-SD 4529 as Tyromyces semisupinus).

Literature Reports: None. The species is new for the BF.

REMARKs. The species is somewhat similar to Antrodiella pallescens (Pilát) Niemelä \& Miettinen, which differs by having narrower and slightly curved basidiospores (ellipsoidal to ovoid in A. faginea) and occurrence on or close to Fomes fomentarius (L.) J. J. Kickx; the predecessors of $A$. faginea are species of hymenochaetoid fungi such as Phellinus spp. and Inonotus spp., more rarely Hymenochaete spp. Moreover, A. faginea often 
produces gloeocystidia, which are not observed in A. pallescens. The species was reported earlier from Poland by Piątek (2001) based on a specimen collected in the Western Carpathians. This specimen in fact represents Antrodiella pallescens (KRAM F-51169). Recently the species was recorded in Kampinos National Park (Karasiński et al. 2015).

\section{Antrodiella foliaceodentata (Nikol.) Gilb.} \& Ryvarden

Fig. $3 \mathrm{H}$

SPeCimens EXAmined: 287A, on fallen trunk of a deciduous tree and Fomes fomentarius dead basidiomata, 21 Sept. 2009, Karasiński 4377 (D.K.); 340B, on Fraxinus excelsior fallen trunk and dead basidiomata of Fomes fomentarius, 16 Sept. 2009, Karasiński 4202, 4203 (D.K.); 340F, on Acer platanoides fallen trunk and dead basidiome of Fomes fomentarius, 20 Sept. 2009, Karasiński 4299 (D.K.); 340, on fallen trunk of Carpinus, 28 Aug. 1973, Wojewoda s.n. (KRAM F-14382); 344B, on Fraxinus excelsior fallen trunk and dead basidiomata of Fomes fomentarius, 11 Aug. 2009, Karasiński 3714 (D.K.); 345A, on Fraxinus excelsior fallen log close to dead basidiome of Fomes fomentarius, 28 July 2009, Karasiński 3598 (D.K.); 370, on fallen trunk of Carpinus, 26 Aug. 1973, Wojewoda s.n. (KRAM F-14347). 372D, on Fraxinus excelsior fallen trunk and dead basidiome of Fomes fomentarius, 20 Aug. 2009, Karasiński 3992 (D.K.); 399A, on fallen trunk of deciduous tree close to dead and living basidiomata of Fomes fomentarius, 1 Nov. 2009, Karasiński 5040 (D.K.).

LiterATURE REPORTS: Domański 1967 (as Irpex foliaceo-dentatus), Kotlaba \& Lazebniček 1967 (as Irpex foliaceo-dentatus), Anonymous 1968 (as Irpex foliaceo-dentatus), Domański 1970d (as Coriolus foliaceo-dentatus), Domański et al. 1973 (as Trametes foliaceo-dentata), Piątek 2001, Niemelä 2013.

REMARKs. Antrodiella foliaceodentata was described from the Caucasus (Nikolajeva 1949) and so far has been found only in the type locality and the BNP. It was not previously reported, but the species seems to be the successor of Fomes fomentarius. For almost all specimens cited above (except those collected by Wojewoda) this association was very conspicuous, and often basidiomata of $A$. foliaceodentata grew directly on dead basidiomata of Fomes fomentarius.

Antrodiella leucoxantha (Bres.) Miettinen \& Niemelä

Fig. 4A

SPecimens eXAmined: 369B, on Carpinus fallen branch, 22 Sept. 2009, Karasiński 4373 (D.K.); BNP, Masiewski Tryb, north of Dziedzinka house, on Carpinus thin fallen trunk, 15 Oct. 2008, Niemelä 8499 (KRA).

LITERATURE REPORT: Niemelä 2013.

REMARKS. This species is characterized by having small, white, effused-reflexed or pileate basidiomata with thin pilei, cylindrical to slightly curved basidiospores, and the absence of a clear connection with fungal predecessors. It is close to Antrodiella pallescens, but the latter is a successor of Fomes fomentarius and has smaller basidiospores. The similar A. faginea has ellipsoidal basidiospores, grows on wood decayed by hymenochaetoid species like Phellinus spp. or Inonotus spp., and often produces gloeocystidia.

\section{Antrodiella pallescens (Pilát) Niemelä} \& Miettinen

Fig. 4B

SPECIMEN EXAMined. 318D, on Betula pendula fallen trunk and Fomes fomentarius dead basidiome, 17 Aug. 2009, Karasiński 3880 (D.K.); BF, on Betula branch, 4 Sept. 1966, Domański (KRAM F-SD 5086 as Tyromyces semisupinus); BF, on hardwood trunk, 10 Aug. 1962, Domański (KRAM F-SD 2219 as Trametes genistae ined.); BF, on Carpinus branch, 4 Aug. 1962, Domański (KRAM F-SD 2498 as Tyromyces semisupinus).

LITERATURE REPORTS: Domański 1965 (as Tyromyces semisupinus), Domański 1967 (as Tyromyces semisupinus), Domański 1972b (as Tyromyces semisupinus), Niemelä 2013, Gierczyk et al. 2014.

Remarks. Among numerous specimens preserved in KRAM F-SD under the name Tyromyces semisupinus, 11 specimens were collected by

Fig. 4. A - Antrodiella leucoxantha (Bres.) Miettinen \& Niemelä, B - Antrodiella pallescens (Pilát) Niemelä \& Miettinen, C Antrodiella romellii (Donk) Niemelä, D - Antrodiella serpula (P. Karst) Spirin \& Niemelä, E - Aporpium canescens (P. Karst.) Bondartsev \& Singer ex Singer, F - Aporpium macroporum Niemelä, Spirin \& Miettinen, G - Aurantiporus croceus (Pers.) Murrill, H - Aurantiporus fissilis (Berk. \& M. A. Curtis) H. Jahn ex Ryvarden. Photo D. Karasiński (A-H). 

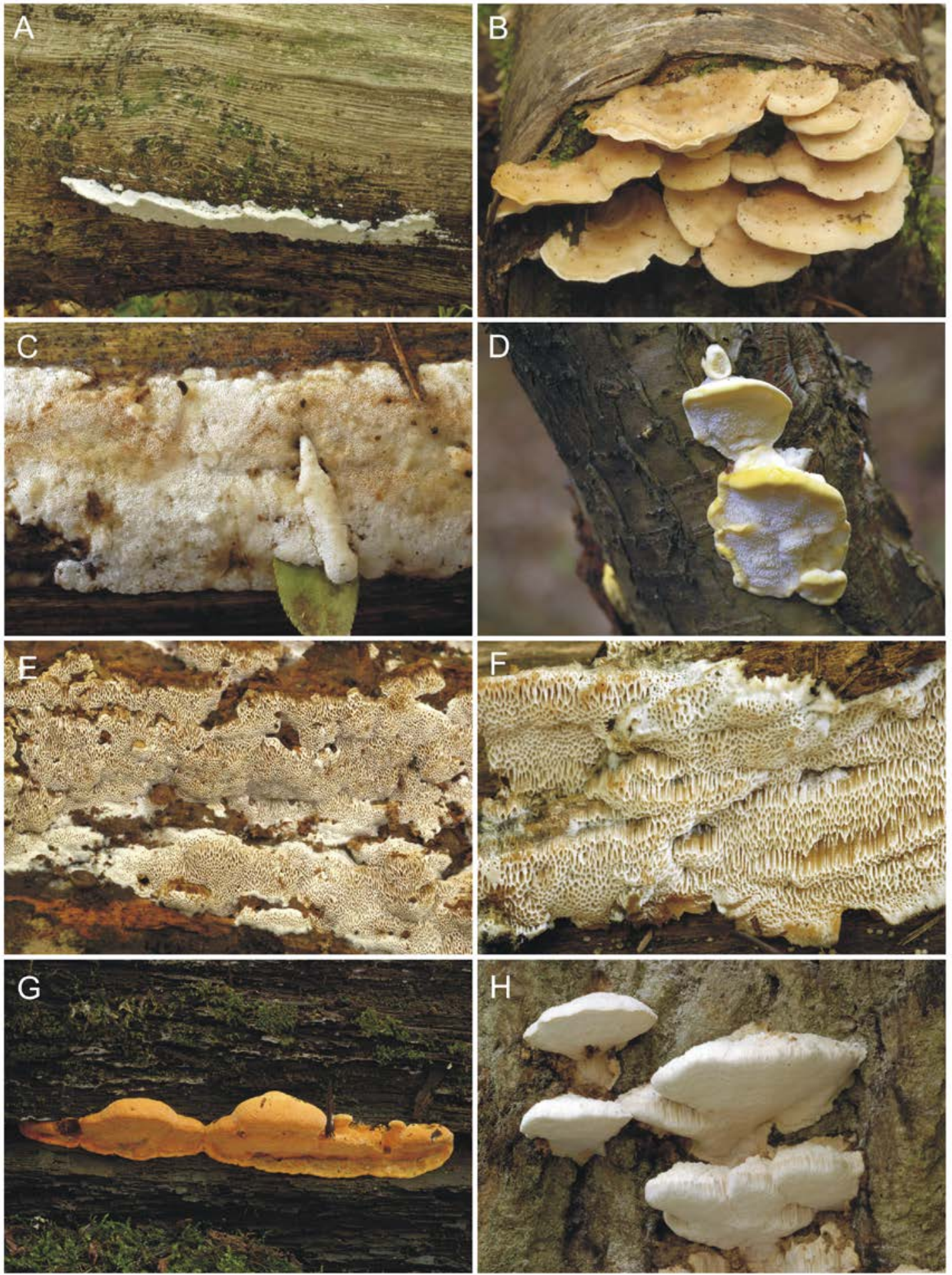
Domański in the BF. These were revised and five different species were identified among them: Antrodiella citrinella, A. faginea, A. pallescens, A. romellii (Donk) Niemelä and Junghuhnia luteoalba (P. Karst.) Ryvarden (for specimen details see under these species).

\section{Antrodiella romellii (Donk) Niemelä Fig. 4C}

Specimen examined. 418 (W. Szafer Landscape Reserve), on bark of Quercus? fallen twig, 29 Sept. 2006, Karasiński 060929/6308 (KRAM F-47250); BF, on Corylus trunk, 5 Aug. 1962, Domański (KRAM F-SD 2495 as Tyromyces semisupinus); BF, on hardwood branch, 6 Aug. 1962, Domański (KRAM F-SD 2496 as Tyromyces semisupinus); BF, on Carpinus trunk, 27 Aug. 1956, Domański (KRAM F-SD 379 as Tyromyces semisupinus); BF, on Betula, 11 Aug.1962, Domański (KRAM F-SD 2497 as Tyromyces semisupinus).

LiteratuRE REPORTS: Domański 1965 (as Tyromyces byssinus), Domański 1967 (as Tyromyces byssinus), Domański 1972b (as Poria romellii), Piątek 2001, Karasiński et al. 2009, Niemelä 2013.

Antrodiella serpula (P. Karst) Spirin \& Niemelä Fig. 4D

SPECIMENS EXAMINED: 282D, on Corylus avellana stump and dead Mensularia radiata, 25 July 2009, Karasiński 3520 (D.K.); 368B, on Corylus stump and Mensularia radiata dead basidiome, 13 Sept. 2009, Karasiński 4078 (D.K.); 399B, on Corylus trunk and Mensularia radiata dead basidome, 2 Oct. 2009, Karasiński 5065 (D.K.); 194D (D.K. 3669C), 225C (D.K. 3687D), 340D (D.K. 3276A).

LiteratURe REPORTS: Domański et al. 1967 (as Trametes hoehnelii), Domański 1967 (as Trametes hoehnelii), Domański 1970b (as Coriolus hoehnelii), Anonymous 1968 (as Trametes hoehnelli), Domański et al. 1973 (as Trametes hoehnelii), Bujakiewicz et al. 1992 (as Antrodiella hoehnelii), Skirgiełło 1997 (as Antrodiella hoehnelii), Bujakiewicz \& Kujawa 2010 (as Antrodiella hoehnelii), Niemelä 2013, Gierczyk et al. 2014 (as Antrodiella hoehnelii).

Antrodiella subradula (Pilát) Niemelä \& Miettinen

SPECIMEN EXAMINED: 342C, on well rotted wood of Acer? or Ulmus? fallen trunk (coexists with Gloeohypochnicium analogum), 17 Oct. 2009, Karasiński 4781 (D.K.).
Literature RePORT: None. The species is new for Poland.

REMARKS. This is the first European find of the species, and probably the second record worldwide. The original specimen was collected on Salix in Siberia (Ziling 233 in PRM, not studied), lectotypified by Donk (1974; see Miettinen et al. 2006 for details). The species is characterized by having resupinate basidiomata with a very thin white subiculum and short cream-colored tubes, angular pores 3-5 per mm, dimitic hyphal structure with skeletal hyphae $2.5-4.5 \mu \mathrm{m}$ wide, and clamped generative hyphae 2.0-3.5 $\mu \mathrm{m}$ wide, short clavate basidia 11-14.5 × 4-5 $\mu \mathrm{m}$ with 4 sterigmata, and ellipsoid, thin-walled, nonamyloid basidiospores, 3-4 × 2.0-2.5 $\mu \mathrm{m}$, often with a few small oil drops inside. Antrodiella romellii has basidiospores similar in shape but larger $(3.5-5.0 \times 2.5-3.2 \mu \mathrm{m})$, and smaller pores (5-7 per $\mathrm{mm}$ ). Macroscopically the species is also somewhat similar to Schizopora radula (Pers.) Hallenb.

Aporpium canescens (P. Karst.) Bondartsev \& Singer ex Singer

Fig. 4E

SPECIMENS EXAMINED: 284, on Betula trunk, 19 Sept. 2011, Karasiński 6344 (D.K.); 285A, on Betula pendula fallen trunk, 19 Sept. 2011, Karasiński 6344 (D.K.); 341D, on Acer platanoides fallen trunk, 17 Oct. 2009, Karasiński 4763 (D.K.); 369C, on fallen trunk of deciduous tree (Ulmus?), 22 Sept. 2009, Karasiński 4387 (D.K.); 402, on Populus fallen trunk, 31 Oct. 2009, Karasiński 4974 (D.K.).

Literature RePORTS: Błoński et al. 1888 (as Polyporus subspadiceus), Domański 1965 (as Aporpium caryae), Domański 1967 (as Aporpium caryae), Domański 1972b (as Aporpium caryae), Wojewoda 1977 (as Aporpium caryae), Wojewoda 1979 (as Aporpium caryae), Niemelä 2013, Gierczyk et al. 2014 (as Protomerulius caryae).

Aporpium macroporum Niemelä, Spirin \& Miettinen

Fig. 4F

SPecimens EXAmined: 370A, on Populus fallen log, 22 Sept. 2009, Karasiński 4400 (D.K.); 402A, on Populus fallen log, 31 Oct. 2009, Karasiński 4964 (D.K.); 402A, abundantly on Populus fallen logs, 31 Oct. 2009, Karasiński 4964, 4971, 4973, 4976 (D.K.). 
LITERATURE REPORTS: Miettinen et al. 2012, Niemelä 2013, Gierczyk et al. 2014.

REMARKs. The species differs from $A$. canescens by having larger pores and wider basidiospores. For more details see Miettinen et al. (2012).

\section{Aurantiporus croceus (Pers.) Murrill Fig. 4G}

Specimens eXamined: 225, on Quercus log, 14 Sept. 2009, Karasiński 4141 (D.K.); 290, on Quercus log, 22 Sept. 2011, Karasiński 6475 (D.K.); 319C, on Quecus log, 28 July 2009, Karasiński 3613 (D.K.); 345B, on Quercus log, 11 Aug. 2009, Karasiński 3718 (D.K.); 346C, on Quercus rotten log, 21 Sept. 2011, Karasiński 6437 (D.K.); 316C (D.K. 5272), 341B (D.K. 5253), 344B (D.K. 3828A), 370C (D.K. 3708A), 373A (H.M.W. M-1870); 373D (D.K. 3428), 373D (D.K. 3448), 374 (D.K. 6437); 375B (D.K. 3572); 417B (H.M.W. M-1431).

Literature REPORTS: Piątek \& Wołkowycki 2005 (as Hapalopilus croceus), Gierczyk et al. 2013 (as Hapalopilus croceus), Niemelä 2013.

Aurantiporus fissilis (Berk. \& M. A. Curtis) H. Jahn ex Ryvarden

Fig. $4 \mathrm{H}$

SPECIMENS EXAMINED: 258C, on Fraxinus excelsior fallen log, 21 Sept. 2009, Karasiński 4359 (D.K.); 340C, on Populus fallen log and stump the same tree, 16 Sept. 2009, Karasiński 4221 (D.K.); 375A, on Populus log, 19 Sept. 2009, Karasiński 4279 (D.K.); 375C, on Populus $\log , 21$ Sept. 2011, Karasiński 6420 (D.K.); 399B, on Populus log, 2 Oct. 2009, Karasiński 5054 (D.K.).

LiterATURE REPORTS: Niemelä 2013.

REMARKs. The similar Spongipellis spumeus (Sowerby) Pat. usually grows solitary and has larger basidiospores.

Aurantiporus priscus Niemelä, Miettinen \& Manninen

Fig. 5A

Specimens examined: 318B, on rotten Pinus fallen trunk, 12 Aug. 2009, Karasiński 3734, 3735, 3736 (D.K.).

LitERATURE REPORTS: Niemelä et al. 2012, Niemelä 2013.

REMARKS. Aurantiporus priscus was recently described based on material from Poland, Finland and Sweden. The type specimen was collected in BNP (Niemelä et al. 2012). This species was previously reported from Europe under the name Hapalopilus salmonicolor (Berk. \& M. A. Curtis) Pouzar or Erastia salmonicolor (Berk. \& M. A. Curtis) Niemelä \& Kinnunen (e.g., Niemelä 2005), but an isotype study of Polyporus salmonicolor Berk. \& M. A. Curtis described from the U.S.A. revealed that the European materials belong to a different species described as Aurantiporus priscus (Niemelä et al. 2012). Hapalopilus salmonicolor is a North American taxon having short cylindrical basidiospores 2.0-2.5 $\mu \mathrm{m}$ wide. Another similar species, Hapalopilus ochraceolateritius (Bondartzev) Bondartzev \& Singer, has narrower basidiospores 1.8-2.2 $\mu \mathrm{m}$ wide. In contrast to these two species, Aurantiporus priscus has broadly ellipsoidal basidiospores, $4-5 \times 2.5-3.5 \mu \mathrm{m}$ in the specimen examined. Hapalopilus salmonicolor reported by Domański (1972b) from two Polish sites outside the Białowieża Forest (Międzyrzec Podlaski and Pojezierze Mazurskie lakeland) in fact represents Hapalopilus aurantiacus (Rostk.) Bondartzev \& Singer with basidiospores 5.0-7.0 $\times 2.5-3.0 \mu \mathrm{m}$. Aurantiporus priscus seems to be a very rare relict species of old-growth and primeval forests, associated with wood of conifers.

\section{Bjerkandera adusta (Willd.) P. Karst.}

SPECIMENS EXAMINED: 98B (D.K. 10986); 344D, on Populus fallen trunk, 28 March 2002, Wolkowycki (H.M.W. M-2799); 385C (Sacharewo), on Betula pendula fallen trunk, 8 Oct. 1998, Wotkowycki (H.M.W. M-1231); 402A (D.K. 10939); 412D, on Carpinus, 5 Nov. 1998, Wotkowycki (H.M.W. M-1353); 413A (H.M.W. M-1212); 437B (H.M.W. M-1884); 487B (H.M.W. M-0984); BF, on deciduous wood, 1 May 1959, Wojewoda s.n. (KRAM F-13473); BF, on hardwood, 1 May 1959, Domański (KRAM F-SD 2648); BF, on Betula fallen branch, Oct. 1955, Domański (KRAM F-SD 2651); BF, on Carpinus, 28 Apr. 1960, Domański (KRAM F-SD 632 as Gloeoporus adustus f. stratosa f.n.); BF, on Carpinus, 10 June 1966, Domański (KRAM F-SD 4991).

Literature RePORTs: Błoński et al. 1888 (as Polyporus adustus), Błoński 1889a (as Polyporus adustus), Siemaszko 1923 (as Polyporus adustus), Nespiak 1956 (as Leptoporus adustus v. carpineus), Orłoś 1960 (as Gloeoporus adustus), Orłoś 1961 (as Gloeoporus 
adustus), Domański et al. 1967 (as Bjerkandera adusta f. carpinea and Bjerkandera adusta f. resupinata), Domański 1967, Anonymous 1968, Domański et al. 1973 (as Bjerkandera adusta f. carpinea and B. adusta f. resupinata), Bujakiewicz et al. 1992, Bujakiewicz 1994, Jaroszewicz 1996, Skirgiełło 1997, Skirgiełło 1998, Szczepkowski et al. 2008, Bujakiewicz \& Kujawa 2010, Gierczyk et al. 2013, Niemelä 2013, Gierczyk et al. 2014.

\section{Bjerkandera fumosa (Pers.) P. Karst.}

Specimens examined: 413B (Sacharewo), on Salix fragilis fallen trunk, 17 Oct. 1997, Wolkowycki (H.M.W. M-1865); BF, Palace Park in Białowieża, on decidous trunk, 21 Oct. 1955, Domański (KRAM F-SD 323); BF, on Populus trunk, 25 Sept. 1965, Domański (KRAM F-SD 4904); BF, on Salix sp. trunk, 26 Sept. 1965, Domański (KRAM F-SD 4923).

LiTERATURE REPORTS: Domański 1967, Anonymous 1968, Niemelä 2013.

\section{Buglossoporus pulvinus (Pers.) Donk Fig. 5B}

SPeCimens EXAmined: 257D, in necrotic depression on trunk of Quercus robur living tree, 21 Sept. 2009, Karasiński 4341 (D.K.); 314A, on Quercus fallen trunk, 7 July 2009, Karasiński 3327 (D.K.); 315A, on Querus fallen branch, 18 Aug. 2009, Karasiński 3895 (D.K.); 315B, on Quercus fallen trunk, 18 Aug. 2009, Karasiński 3908 (D.K.); 316B, on strongly decayed Quercus trunk, 14 Aug. 2009, Karasiński 3793, 3797 (D.K.); 318D, on Quercus robur rotten trunk, 17 Aug. 2009, Karasiński $3862 b$ (D.K.); 369A, on rotten Quercus fallen trunk, 14 July 2009, Karasiński 3486 (D.K.); 373D, on Quercus fallen trunk, 11 July 2009, Karasiński 3429, 3451 (D.K.); 374C, on Quercus fallen trunk, 11 July 2009, Karasiński 3423 (D.K.); 374C, on Quercus fallen trunk, 26 July 2009, Karasiński 3545 (D.K.); 374D, on Quercus fallen trunk, 28 July 2009, Karasiński 3590 (D.K.); 375B, on Quercus big log, 27 July 2009, Karasiński 3573 (D.K.).

Literature REPORTs: Domański et al. 1967 (as Piptoporus quercinus), Domański 1967 (as Piptoporus quercinus), Domański et al. 1973 (as Piptoporus quercinus), Niemelä 2013.
REMARKS. Basidiomata of this species appear in summer and rarely at the beginning of autumn. In the study area all specimens except one were found on fallen logs, trunks and branches of Quercus in early or middle stages of wood decomposition. Only once it was observed in a necrotic depression $\mathrm{ca} 1 \mathrm{~m}$ above ground level on the trunk of a living old Quercus tree. These observation conflict with literature data in which the species is usually described as a parasite on living trees and most rarely a saprobe on dead wood (e.g., Domański et al. 1973; Ryvarden \& Gilbertson 1994; Bernicchia 2005; Ryvarden \& Melo 2014).

\section{Ceriporia aurantiocarnescens (Henn.) M. Pieri} \& B. Rivoire

\section{LITERATURE REPORTS: Niemelä 2013.}

REMARKS. The species was reported by Niemelä (2013) without a precise locality. We did not find it in the BF.

Ceriporia excelsa (S. Lundell) Parmasto

Fig. 5C

SPeCimens EXAMINED: 340A, on rotten Quercus log, 22 Sept. 2010, Karasiński 6001 (D.K.).

LITERATURE REPORTS: Niemelä 2013.

REMARKS. This species is characterized by having a pink to reddish orange pore surface and oblong to short cylindrical basidiospores. It is a rare species in the study area and also in Poland (Wojewoda 2003).

Ceriporia purpurea (Fr.) Donk

Fig. 5D

SPECIMENS EXAMINED: 314C, on Fraxinus excelsior fallen branch, 25 Apr. 2010, Karasiński $5182 b$ (D.K.); 340F, on Ulmus fallen branch, 22 Sept. 2009, Karasiński 4415 (D.K.).

LiterATURE REPORTS: Domański 1967 (as Meruliopsis purpurea), Niemelä 2013.

Fig. 5. A - Aurantiporus priscus Niemelä, Miettinen \& Manninen, B - Buglossoporus pulvinus (Pers.) Donk, C - Ceriporia excelsa (S. Lundell) Parmasto, D - Ceriporia purpurea (Fr.) Donk, E - Ceriporia viridans (Berk. \& Broome) Donk, F - Ceriporiopsis aneirina (Sommerf.) Domański, G - Ceriporiopsis resinascens (Romell) Domański, H - Cerrena unicolor (Bull.) Murrill. Photo D. Karasiński (A-H). 

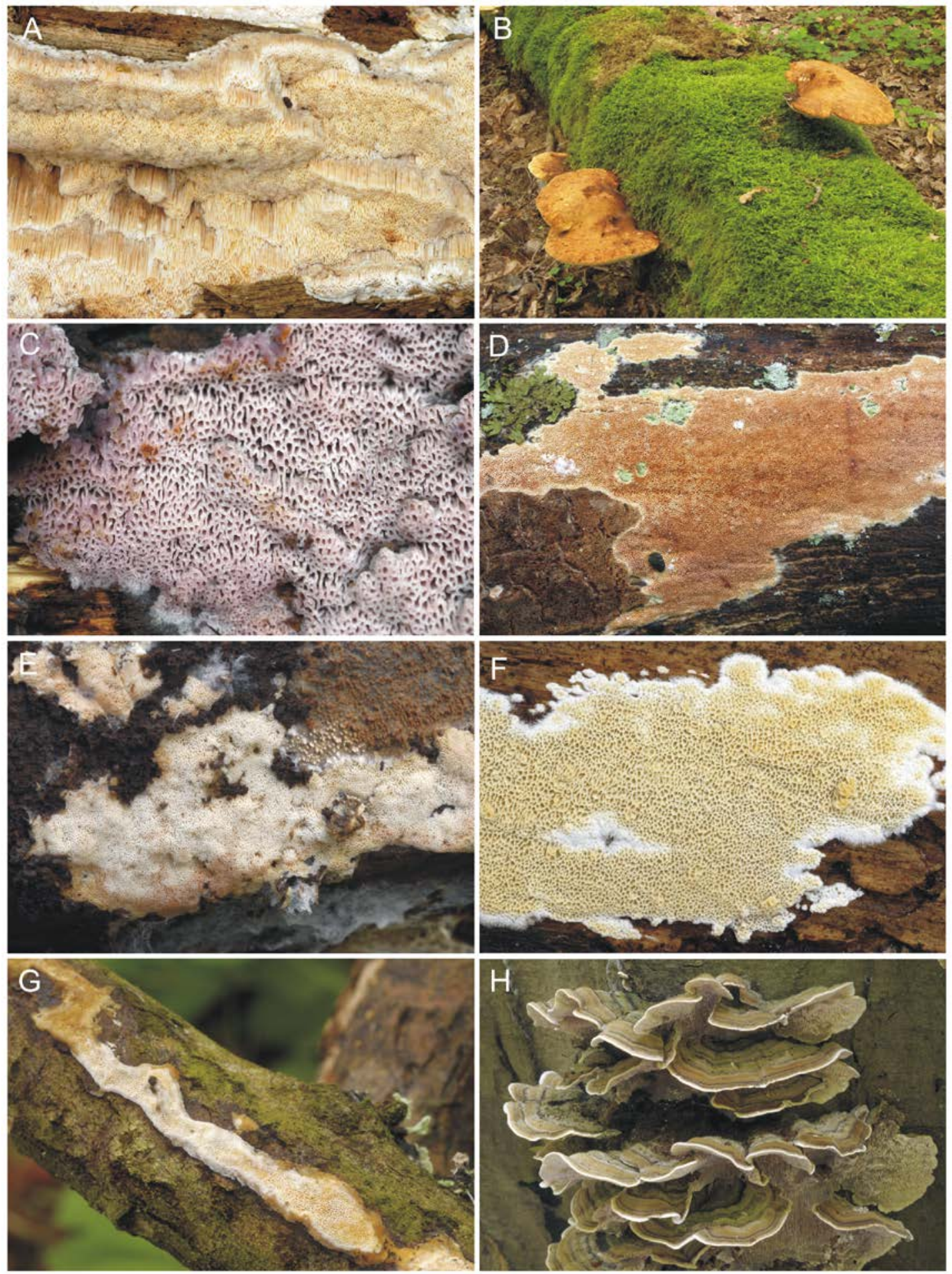
REMARKS. This species has basidiomata similar to $C$. excelsa but differs in having slighty smaller pores and larger, allantoid basidiospores.

Ceriporia viridans (Berk. \& Broome) Donk Fig. 5E

Specimen examined: 398, on Carpinus fallen trunk, 21 Sept. 2007, Karasiński 1671 (D.K.); BF, on Corylus avellana trunk, 10 Aug. 1962, Domański (KRAM F-SD 2310); BF, on wood of Tilia sp., 12 June 1966, Domański (KRAM F-SD 5015); BF, on wood of Tilia sp., 22 Sept. 1965, Domański (KRAM F-SD 4865); BF, on dead basidiocarp of Fomes fomentarius, 9 Aug. 1962, Domański (KRAM F-SD 2542); BF, on wood of Betula sp., 23 Oct. 1957, Domański (KRAM F-SD 5444).

Literature RePOrTs: Błoński et al. 1888 (as Polyporus viridans), Domański 1965, 1967, 1972b, Niemelä 2013.

\section{Ceriporiopsis aneirina (Sommerf.) Domański}

Fig. 5F

Specimens eXAmined: 316A, on Populus log, 27 Apr. 2010, Karasiński 5276 (D.K.); 370B, on Populus fallen branch, 17 Oct. 2009, Karasiński 4799 (D.K.); 399A, on Populus fallen branch, 1 Nov. 2009, Karasiński 5043 (D.K.), 402A, on Populus fallen trunk, 31 Oct. 2009, Karasiński 4958 (D.K.); 402A (D.K. 4969A); 418C (D.K. 4568).

LITERATURE REPORTS: Domański 1963, 1965, 1967, 1972b, Karasiński et al. 2009, Niemelä 2013, Gierczyk et al. 2014.

\section{Ceriporiopsis resinascens (Romell) Domański}

Fig. 5G

Specimens examined: 402A, on Populus trunk, 13 Sept. 2013, Karasiński 10092 (D.K.); BF, on Alnus trunk, 27 Oct. 1956, Domański (KRAM F-SD 568); BF, on Salix sp. branch, 23 Sept. 1965, Domański (KRAM F-SD 4883).

\section{LITERATURE REPORTS: Domański 1967.}

REMARKS. This uncommon species is macroscopically similar to $C$. aneirina but usually forms smaller basidiomata with smaller pores 3-4 per $\mathrm{mm}$ (1-3 per $\mathrm{mm}$ in C. aneirina), and narrower, cylindrical basidiospores (broadly ellipsoidal in C. aneirina).
Cerrena unicolor (Bull.) Murrill

Fig. $5 \mathrm{H}$

SPECIMENS EXAMINED: 130, on Betula pendula fallen trunk, 14 Sept. 2009, Karasiński 4109 (D.K.); 225B, on Betula fallen trunk, 11 Oct. 2009, Karasiński 4587 (D.K.); 272D (H.M.W. M-1107); 314A, on Populus fallen trunk, 7 July 2009, Karasiński 3300 (D.K.); 375D (D.K. 10973); 384C (H.M.W. M-1132); 413A (H.M.W. M-1217); 488C (H.M.W.M-0963); 500A (H.M.W. M-2258); 516D (H.M.W. M-2132); 668D (H.M.W. M-1113).

LITERATURE REPORTS: Błoński et al. 1888 (as Daedalea unicolor), Błoński 1889a (as Daedalea unicolor), Siemaszko 1925 (as Daedalea unicolor), Domański 1967, Anonymous 1968, Domański et al. 1973 (as Cerrena unicolor f. irpicoides), Bujakiewicz et al. 1992, Jaroszewicz 1996, Skirgiełło 1997, Szczepkowski et al. 2008, Gierczyk et al. 2013, Niemelä 2013, Gierczyk et al. 2014.

\section{Chaetoporellus latitans (Bourdot \& Galzin)} Bondartsev \& Singer ex Singer

Specimens EXAmined: BF, on wood of Picea fallen trunk, 20 Sept. 1960, Domański (KRAM F-SD 845).

Literature REPORTS: Domański 1965, Domański 1967, Domański 1972b, Niemelä 2013.

REMARKS. In the BF it seems to be a very rare species, not found during recent fieldwork. The specimen cited above was previously reported by Domański (1965, 1967, 1972b). Niemelä (2013) reported it from the $\mathrm{BF}$ based on literature data (after Domański 1972b).

Cinereomyces lindbladii (Berk.) Jülich Fig. 6A

SPECimens eXamined: 135C (D.K. 3339D); 135D, on Pinus fallen trunk, 8 July 2009, Karasiński 3337 (D.K.); 194C (D.K. 3358A); 225B, on Picea fallen trunk, 14 Sept. 2009, Karasiński 4142 (D.K.); 260A (D.K. 4643); 261A (D.K. 3375A, DK 3380A); 285A (D.K. 5309; DK 5314); 319C (D.K. 3757), 340C, on Picea fallen log, 6 July 2009, Karasiński 3284 (D.K.); 340F (D.K. 4311); 346D (D.K. 4696A); 369B (D.K. 4198); 373C, on Quercus fallen trunk, 31 Oct. 2009, Karasiński 4982 (D.K.); 373D (D.K. 3460A2); 374C (D.K. 4158); 399C (D.K. 5028A); 412B (H.M.W. M-1947); 414C (H.M.W. M-2815); 415C (H.M.W. M-1469); 463C (H.M.W. M-1284); 500A (H.M.W. M-1520).

Literature RePorts: Domański 1959a (as Poria cinerascens), Domański 1965 (as Tyromyces cinera- 
scens), Domański 1967 (as Tyromyces cinerascens), Domański 1972b (as Poria cinerascens), Piątek 2002b, 2003, Szczepkowski et al. 2008, Niemelä 2013.

REMARKS. This species appears to be common in the study area.

\section{Climacocystis borealis (Fr.) Kotl. \& Pouzar}

Fig. 6B

Specimens examined: 194D, on Picea stump, 30 July 2009, Karasiński 3670 (D.K.), 224B (D.K. 3932E); 225C, on Picea stump, 30 July 2009, Karasiński 3687H (D.K.); 254D (D.K. 3808B); 313B, on Picea dead standing trunk, 25July 2009, Karasiński 3517 (D.K.); 314B (D.K. 3885A); 318D (D.K. 3880A); 319C, on Picea stump, 13 Aug. 2009, Karasiński 3760 (D.K.); 340A (D.K. 4322A); 368A (D.K. 4080), 369B (D.K. 4199A); 370C, on Picea fallen log, 10 Aug. 2009, Karasiński 3703F (D.K.); 374D (D.K. 10947); 412B (H.M.W. M-2850); 413D (H.M.W. M-1896); 439A (H.M.W. M-1258); 486A (H.M.W. M-1879); 488C (Nieznanowo reserve), on Picea stump, 13 Sept. 2005, Karasińśki 050913-7544 (D.K.).

Literature REPORTS: Błoński et al. 1888 (as Polyporus borealis), Siemaszko 1925 (as Polyporus borealis), Pilát 1950 (as Leptoporus borealis), Orłoś 1951 (as Polyporus borealis), Benedix 1967 (as Spongipellis borealis), Orłoś 1955b (as Polyporus borealis), Orłoś 1960 (as Leptoporus borealis), Orłoś 1961 (as Leptoporus borealis), Domański et al. 1967 (as Climacocystis borealis var. borealis and C. borealis var. spathulatus), Domański 1967, Anonymous 1968, Domański et al. 1973 (as Climacocystis borealis var. borealis and $C$. borealis var. spathulatus), Bujakiewicz et al. 1992, Skirgiełło 1997, Bujakiewicz 2003, Szczepkowski et al. 2008, Bujakiewicz \& Kujawa 2010, Gierczyk et al. 2013, Niemelä 2013, Gierczyk et al. 2014.

\section{Coltricia cinnamomea (Jacq.) Murrill Fig. 6C}

SPECIMEN EXAMINED: 285A, on the ground under Picea, Carpinus and Quercus, 15 Aug. 2009, Karasiński $3802 b$ (D.K.).

LITERATURE REPORTS: Kotlaba \& Lazebniček 1967, Anonymous 1968, Gierczyk et al. 2013, Niemelä 2013.

REMARKs. This species is rare in the studied area.

\section{Coltricia perennis (L.) Murrill}

SPECIMENS EXAMINED: 383D, on mineral soil in Serratulo-Pinetum, 20 Aug. 2000, Wotkowycki (H.M.W.
M-0983); 384C (H.M.W. M-0982); 385D (H.M.W. M-1369); 417 (W. Szafer Landscape Reserve), on the ground, 29 Sept. 2006, Karasiński 060929-6310 (D.K.); 487B (H.M.W. M-1405); 596A (H.M.W. M-1743); 666A, on mineral soil in Peucedano-Pinetum, 27 Aug. 2000, Wolkowycki (H.M.W.M-1036); 668A (H.M.W. M-1037); 698 (Starzyna Reserve), on sandy soil, 28 Sept. 2006, Karasiński 060928-6112 (D.K.).

Literature RePORTS: Błoński et al. 1888 (as Polyporus perennis), Błoński 1889a (as Ochroporus perennis), Siemaszko 1925 (as Polyporus perennis), Nespiak 1959 (as Xanthochrous perennis), Orłoś 1960 (as Polystictus perennis), Orłoś 1961 (as Polystictus perennis), Anonymous 1968, Nespiak 1968, 1970, Bujakiewicz et al. 1992, Jaroszewicz 1996, Skirgiełło 1997, Niemelä 2013, Gierczyk et al. 2014.

REMARKS. Recent records of this species are only outside the BNP borders.

Coriolopsis gallica (Fr.) Ryvarden

Fig. 6D

Specimens exAmined: 194C, on Fraxinus fallen trunk, 9 July 2009, Karasiński 3367 (D.K.); 194D (D.K. 3668); 194D (D.K. 3367); 214D (D.K. 11014); 225B (D.K. 3687); 253D (D.K. 4099B); 254D (D.K. 3814F); 314C, on Fraxinus fallen branch, 25 Apr. 2010, Karasiński 5182 A (D.K.); 314C, on Fraxinus fallen log, 25 Apr. 2010, Karasiński 5193 (D.K.); 334C, on bark of Fraxinus fallen trunk, 24 March 1993, Wotkowycki (H.M.W. M-2259); 370C (D.K. 4720); 373D (H.M.W. M-0051); 413A (H.M.W. M-3450); 439C (H.M.W. M-1409); 487A (H.M.W. M-2822); 488C (Nieznanowo Reserve), on Fraxinus fallen trunk, 13 Sept. 2005, Karasińśki 050913-7611 (D.K.); 572 (Michnówka Reserve), on Fraxinus fallen trunk, 24 May 2006, Karasiński 060524-5627 (D.K.).

LITERATURE REPORTS: Orłoś 1960 (as Trametes gallica), Domański et al. 1967 (as Funalia extenuata and F. extenuata f. resupinato-reflexa), Domański 1968 (as Trametella extenuata), Domański et al. 1973 (as Trametella extenuata), Bujakiewicz et al. 1992 (as Coriolopsis extenuata), Bujakiewicz 1994 (as Coriolopsis extenuata), Skirgiełł 1997 (as Coriolopsis extenuata), Bujakiewicz 2002, 2003, Szczepkowski et al. 2008, Bujakiewicz \& Kujawa 2010, Niemelä 2013 (as Trametella gallica), Gierczyk et al. 2014.

\section{Coriolopsis trogii (Berk.) Domański Fig. 6E}

Specimens exAmined: 214D (Szczekotowo Reserve), on Populus big fallen log, 17 Oct. 2014, 
Karasiński 11016 (D.K.); 318D, on Populus fallen trunk, 10 March 2002, Wotkowycki (H.M.W. M-2905); 373C, on Populus fallen branch, 20 Aug. 2009, Karasiński 3975 (D.K.); 402B, on Populus fallen branch, 13 July 2009, Karasiński 3479 (D.K.); 417 (W. Szafer Landscape Reserve), on Populus fallen trunk, 29 Sept. 2006, Karasiński 060929-6335 (D.K.).

LiteratURE REPORTS: Szczepkowski et al. 2008, Niemelä 2013 (as Trametella trogii), Gierczyk et al. 2014.

REMARKS. This species probably is uncommon in the study area or else is undercollected.

\section{Daedalea quercina (L.) Pers.}

Fig. 6F

Specimens examined: 98B, on Quercus stump, 17 Oct. 2014, Karasiński 10990 (D.K.); 413A, on Quercus fallen trunk, 5 Oct. 1998, Wolkowycki (H.M.W. M-1210); 413B (H.M.W. M-1423); 580 (Podcerkwa), on Quercus, 1 Sept. 1973, Wojewoda s.n. (KRAM F-58156); 703A (H.M.W. M-1121); BF, on Quercus, 25 Sept.1965, Domański (KRAM F-SD 4906); BF, on bark of Quercus trunk, 30 Aug. 1957, Domański (KRAM F-SD 461 as Trametes quercina $\mathrm{f}$. lenzitoidea).

LITERATURE REPORTS: Błoński et al. 1888, Siemaszko 1925, Karpiński 1949, Nespiak 1956, Orłoś 1960 (as Trametes quercina), Orłoś 1961 (as Trametes quercina), Domański et al. 1967, Domański 1967, Anonymous 1968, Nespiak 1968, Domański et al. 1973, Bujakiewicz et al. 1992, Jaroszewicz 1996, Skirgiełło 1997, Szczepkowski et al. 2008, Gierczyk et al. 2013, Niemelä 2013.

Daedaleopsis confragosa (Bolton) J. Schröt.

Fig. 6G

Specimens examined: 214D (Szczekotowo Reserve), on Alnus fallen trunk, 17 Oct. 2014, Karasiński 11023 (D.K.); 385C (H.M.W. M-1365); 412B (H.M.W. M-0967); 413D (Sacharewo), on trunk of Salix caprea, 25 Sept. 1999, Wołkowycki (H.M.W. M-1887); 414C (H.M.W. M-1930); 437A (H.M.W. M-3596); 438A, on Alnus glutinosa, 11 Oct. 1998 (H.M.W. M-1279); 439A (H.M.W. M-1129); 572A (H.M.W. M-1130); 580 (Podcerkwa), on fallen branch of deciduous tree, 1 Sept. 1973, Wojewoda s.n. (KRAM F-58157); 601C (H.M.W. M-1117); 702B (H.M.W. M-1144); BF, on Alnus, 8 Aug. 1962, Domański (KRAM F-SD 3650); BNP, NE of the main gate, on Salix caprea branches along forest road, 10 Oct. 2008, Niemelä 8430 (KRAM F-47906).

LITERATURE REPORTS: Błoński et al. 1888 (as Trametes rubescens), Błoński 1889a (as Daedalea rubescens var. anceps), Orłoś 1951 (as Trametes rubescens), Orłoś 1960 (as Trametes confragosa), Domański et al. 1967, Domański 1967, Anonymous 1968, Domański et al. 1973 (as Daedaleopsis confragosa f. anceps), Bujakiewicz et al. 1992, Bujakiewicz 1994, Jaroszewicz 1996, Skirgiełło 1997, Wojewoda 2002 (as Daedaleopsis tricolor), Szczepkowski et al. 2008, Bujakiewicz \& Kujawa 2010, Gierczyk et al. 2013, Niemelä 2013, Gierczyk et al. 2014.

\section{Datronia mollis (Sommerf.) Donk}

Fig. $6 \mathrm{H}$

Specimens EXAmined: 98B (D.K. 10988); 214D (D.K. 11020); 369A, on Populus fallen branch, 14 July 2009, Karasiński 3480D (D.K.); 370C, on Acer fallen branch, 4 July 2009, Karasiński 3228B (D.K.); 372B, on Populus log, 18 Aug. 2009, Karasiński 3978 (D.K.); 372D, on Populus fallen trunk, 20 Aug. 2009, Karasiński $3978 b$ (D.K.); 412C (H.M.W. M-1357); 413C (H.M.W. M-2162); 414B (H.M.W. M-1950); 415A (H.M.W. M-3419); 437B (H.M.W. M-1908); 438A (H.M.W. M-1282); 516C (H.M.W. M-2130); 580 (Podcerkwa), on fallen trunk, 1 Sept. 1973, Wojewoda s.n. (KRAM F-58158).

LiterATURE REPORTS: Orłoś 1960 (as Trametes mollis), Orłoś 1961 (as Trametes mollis), Domański 1965 (as Antrodia mollis), Domański 1967 (as Antrodia mollis), Anonymous 1968 (as Antrodia mollis), Domański 1972b, Bujakiewicz et al. 1992, Skirgiełło 1997, Szczepkowski et al. 2008, Gierczyk et al. 2013, Niemelä 2013, Gierczyk et al. 2014.

REMARKs. This species appears to be common in Poland (Wojewoda 2003) but uncommon in the $\mathrm{BF}$.

Dichomitus albidofuscus (Domański) Domański Fig. 7A

Specimens eXAmined: BF, on Picea fallen trunk, 10 Aug. 1965, Domański 4541 (KRAM F-SD 4541-holotype); 130C, on rotten Picea fallen trunk, 14 Sept. 2009, Karasiński 4120 (D.K.); 261A, on bark of Picea fallen trunk, 10 July 2009, Karasiński 3378 (D.K.); 284D,

Fig. 6. A - Cinereomyces lindbladii (Berk.) Jülich, B - Climacocystis borealis (Fr.) Kotl. \& Pouzar, C - Coltricia cinnamomea (Jacq.) Murrill, D - Coriolopsis gallica (Fr.) Ryvarden, E - Coriolopsis trogii (Berk.) Domański, F - Daedalea quercina (L.) Pers., G - Daedaleopsis confragosa(Bolton) J. Schröt., H - Datronia mollis (Sommerf.) Donk. Photo D. Karasiński (A-H). 

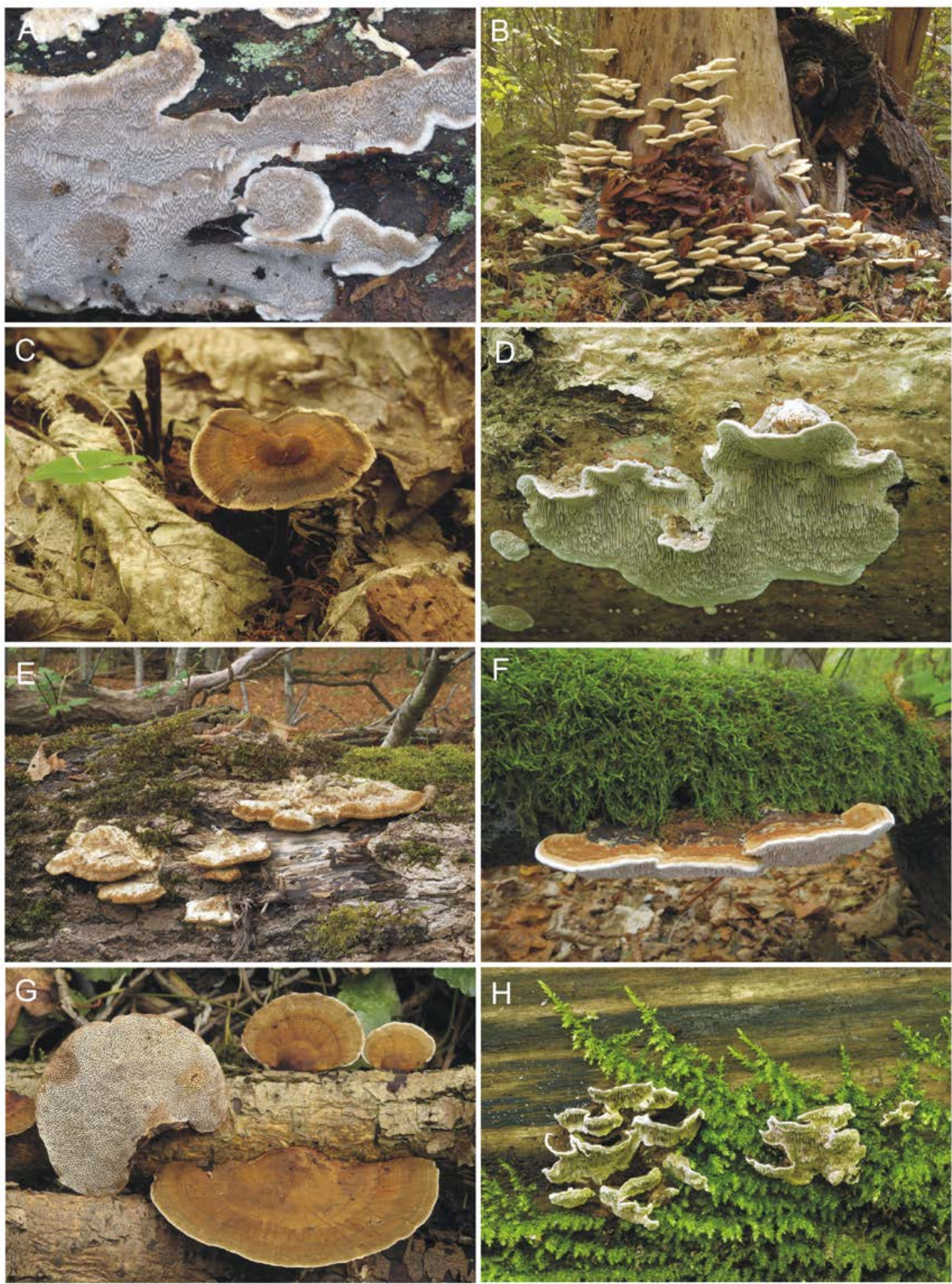
on bark of Picea fallen trunk, 7 July 2009, Karasiński 3320 (D.K.); 285C, on Picea fallen trunk, 28 Apr. 2010, Karasiński 5330d (D.K.); 316B, on Picea fallen log, 27 Apr. 2010, Karasiński 5254 (D.K.); 318C, on Picea fallen trunk, 16 Aug. 2009, Karasiński 3840 (D.K.); 340F, on Picea fallen branch, 6 July 2009, Karasiński 3285 (D.K.); 373C, on Picea fallen log, 13 July 2009, Karasiński $3455 a$ (D.K.); 374C, on Picea fallen trunk, 11 July 2009, Karasiński 3402 (D.K.); 374D, on Picea fallen log, 26 July 2009, Karasiński 3536 (D.K.); 375A, on Picea fallen log, 13 July 2009, Karasiński 3455 a (D.K.); 375B, on Picea fallen trunk, 19 Sept 2009, Karasiński 4269a (D.K.); 399B, on Picea fallen trunk, 5 July 2009, Karasiński 3269 (D.K.); 401B, on Picea fallen trunk, 24 June 2008, Karasiński 1717 (D.K.).

Literature REPORTs: Domański 1966 (as Poria albidofusca), Domański 1967 (as Poria albidofusca), Domański 1972b, Niemelä 2013.

REMARKs. The species has its locus classicus in the BF (Domański 1966). Its basidiomata develop on wood at the end of spring or in summer, and occasionally later. The earliest basidiomata in the initial stage of developement were observed at the end of April, after a mild and almost snowless winter. Usually the basidiomata mature in June or the beginning of July, and later disappear quickly. When the summer has a long rainless period, some dead, dry remnants of basidiomata can be found also in August and even September, but they are difficult to identify in this condition. The species is known so far from BNP, a few localities in the Czech Republic and the European part of Russia (Vampola \& Vlasák 1992; Kotkova \& Isaeva 2007). Based on molecular data a new combination into Donkiporia Kotl. \& Pouzar has recently been proposed for this species (Vlasák et al. 2010).

Dichomitus campestris (Quél.) Domański \& Orlicz Fig. 7B

SPECIMENS EXAMINED: 399A, on dead still-attached branch of Corylus avellana, 4 July 2009, Karasiński 3240 (D.K.).
Literature REPORTS: None. This species is new for BNP.

REMARKS. In the study area the species appears to be very rare, so far known from only one specimen, found close to the main gate to the Park. The species is associated with Corylus avellana dead standing trunks and dead still-attached branches. In the south of Poland it was recorded also on Alnus incana.

Diplomitoporus crustulinus (Bres.) Domański

Fig. 7C

Specimens EXAmined: BF, on Picea fallen branch, 4 Aug. 1965, Domański (KRAM F-SD 4473).

LiterATURE REPORTs: Domański 1970a, Domański 1972b, Niemelä 2013.

REMARKs. This species is known in the study area from one specimen cited above, and was not confirmed in recent fieldwork. Niemelä (2013) reported it from the $\mathrm{BF}$ based on literature data (after Domański 1970a).

Diplomitoporus flavescens (Bres.) Domański

Fig. 7D

Specimens EXAmined: 317C, on Pinus fallen trunk, 14 Aug. 2009, Karasiński 3786 (D.K.); 725 (Krugłe), on Pinus dead standing trunk, 31 Oct. 2014, Wołkowycki (H.M.W. M-3263).

Literature RePORTS: Domański 1970a, Szczepkowski et al. 2008, Niemelä 2013, Gierczyk et al. 2014.

REMARKs. This species probably is not rare in the study area but is undercollected.

Fibroporia gossypium (Speg.) Parmasto Fig. 7E

Specimens examined: 135C, on Pinus fallen log, 8 July 2009, Karasiński 3344 (D.K.); 225A (D.K. 4138); 226A, on bark of Alnus glutinosa fallen trunk, 9 July 2009, Karasiński 3355A (D.K.); 254D (D.K. 3807); 256B (D.K. 3629); 272 (Lipiny Reserve), on Picea fallen trunk, 26 Sept 2006, Karasiński 060926-5853 (D.K.); 285A (D.K. 3801); 288D, on Picea rotten trunk, 12 Aug.

Fig. 7. A - Dichomitus albidofuscus (Domański) Domański, B - Dichomitus campestris (Quél.) Domański \& Orlicz, C - Diplomitoporus crustulinus (Bres.) Domański (dried specimen KRAM F-SD 4473), D - Diplomitoporus flavescens (Bres.) Domański, E - Fibroporia gassypium (Speg.) Parmasto, F - Fistulina hepatica (Schaeff.) Fr., G - Fomitiporia punctata (Fr.) Murrill, H - Fomitiporia robusta (P. Karst.) Fiasson \& Niemelä. Photo D. Karasiński (A-H). 

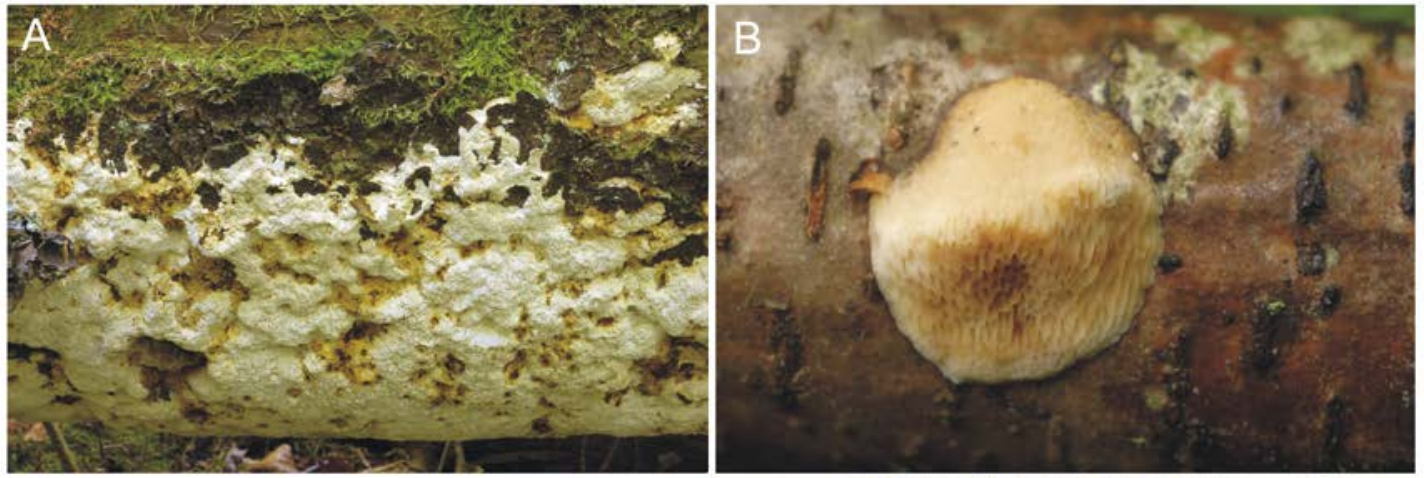

\section{C}
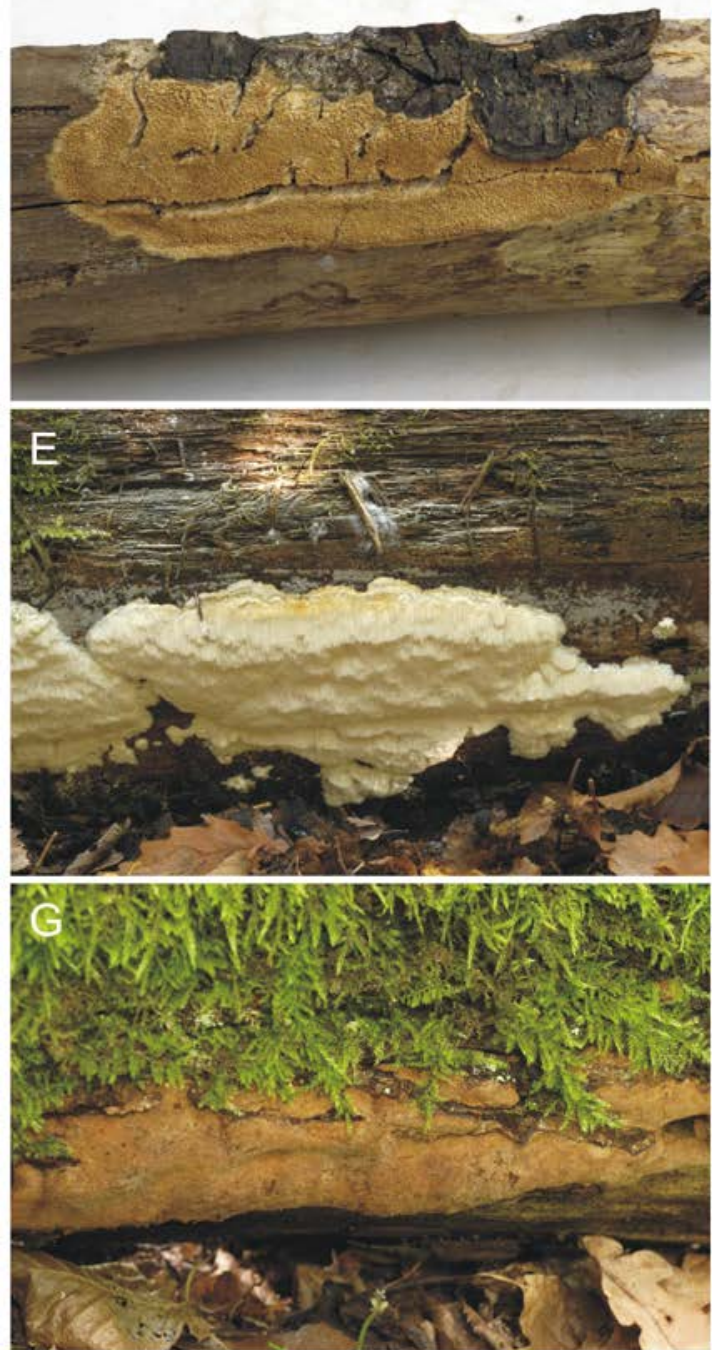

D
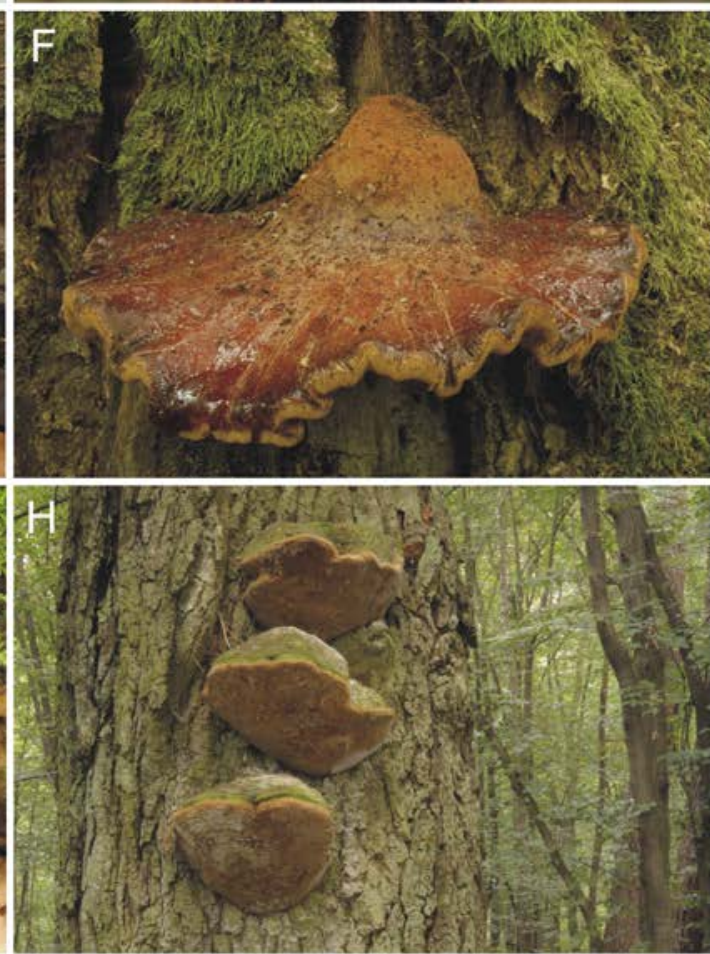
2009, Karasiński 3737 (D.K.); 313B (D.K. 3511); 314B (D.K. 3887); 315B (D.K. 3904); 315B (D.K. 3917A); 318D (D.K. 3859); 340C (D.K. 4239A); 340F (D.K. 4314); 341C (D.K. 4409); 342C (D.K. 4793); 345A (D.K. 3716A); 345B (D.K. 3718D); 369A (D.K. 3488); 372B, on Picea big log, 20 Aug. 2009, Karasiński 3984b (D.K.); 372B (D.K. 3986B); 373C (D.K. 3458); 374A (D.K. 4171); 375B (D.K. 4654).

Literature RePORTs: Domański 1965 (as Tyromyces resupinatus), Domański 1967 (as Tyromyces resupinatus), Domański 1972b (as Fibroporia gossypia), Szczepkowski et al. 2008 (as Antrodia gossypina), Szczepkowski et al. 2010 (as Antrodia gossypina), Niemelä 2013, Gierczyk et al. 2014 (as Antrodia gossypium).

REMARKS. This species appears to be common in the study area.

Fistulina hepatica (Schaeff.) Fr.

Fig. 7F

Specimens eXAmined: 254D, on dead standing Quercus trunk, 15 Aug. 2009, Karasiński 3800C (D.K.); 258C, on trunk of Quercus living tree ca 280 years old, Karasiński 4353 (D.K.); 318D (D.K. 3747); 318D (D.K. 3862); 319C, at base of living Quercus, 11 Aug. 2009, Karasiński 3719 (D.K.); 340C (D.K. 4319B); 374D (D.K. 3731); 402 (D.K. 3965); 402B (D.K. 3827); 414B (H.M.W. M-1658).

LiTERATURE REPORTS: Błoński et al. 1888, Błoński 1889a, Karpiński 1949, Orłoś 1955b, 1960, 1961, Domański 1967, Anonymous 1968, Nespiak 1968, Nespiak 1970, Skirgiełło 1970, Bujakiewicz et al. 1992, Jaroszewicz 1993, 1996, Skirgiełło 1997, Bujakiewicz 2003, Szczepkowski et al. 2008, Bujakiewicz \& Kujawa 2010, Szczepkowski et al. 2011, Gierczyk et al. 2013, Niemelä 2013, Gierczyk et al. 2014.

\section{Fomes fomentarius (L.) J. J. Kickx}

Specimens examined: 98B, on Populus trunk, 17 Oct.2014, Karasiński 10983 (D.K.); 214D (D.K. 11001); 287A (D.K. 4377A); 340B (D.K. 4203A); 340F (D.K. 4299A); 344B (D.K. 3714A); 345A, on Fraxinus log, 11 Aug. 2009, Karasiński 3714 (D.K.); 345A (D.K. 3598A); 372D (D.K. 3992A); 385C (H.M.W. M-0973); 399A (D.K. 5040A); 402A (D.K. 10940); 513B (H.M.W. M-1109).

Literature RePORTS: Błoński et al. 1888 (as Polyporus fomentarius), Błoński 1889a (as Ochroporus fomentarius), Siemaszko 1925 (as Polyporus fomentarius), Karpiński 1949 (as Polyporus fomentarius),
Orłoś 1951, 1955a, b, Nespiak 1956, Orłoś 1960, 1961, Orłoś \& Twarowska 1967, Domański et al. 1967, Domański 1967, Anonymous 1968, Nespiak 1968, Skirgiełło 1976, Nespiak 1970, Bujakiewicz et al. 1992, Bujakiewicz 1994, Jaroszewicz 1996, Grzywacz et al. 1996, Skirgiełło 1997, 1998, Szczepkowski et al. 2008, Bujakiewicz \& Kujawa 2010, Szczepkowski et al. 2011, Gierczyk et al. 2013, Niemelä 2013, Gierczyk et al. 2014.

REMARKS. This is a widely distributed temperate and boreal species, common in the study area.

Fomitiporia punctata (Fr.) Murrill Fig. 7G

Specimens eXAmined: 402A, on bark of Populus fallen trunk, 19 Sept. 2013, Karasiński 9961 (D.K.); 412A (Sacharewo), on Prunus avium fallen branch, 26 March 2002, Wolkowycki (H.M.W. M-2720); 412B (H.M.W. M-1511); 463A (H.M.W. M-1288); BF, on Corylus avellana trunk, 18 Sept. 1960, Domański (KRAM F-SD 1101); BF, on Populus trunk, 24 Oct. 1955, Domański (KRAM F-SD 514).

LITERATURE REPORTS: Domański 1965, 1967, 1972b, Szczepkowski et al. 2008, Niemelä 2013. All literature reports as Phellinus punctatus.

Fomitiporia robusta (P. Karst.) Fiasson \& Niemelä Fig. $7 \mathrm{H}$

SPECIMENS EXAMINED: 225, on trunk of Querus old living tree, 20 Sept. 2011, Karasiński 6394 (D.K.); 412B, on trunk of Quercus living tree, 29 Nov. 2000, Wotkowycki (H.M.W. M-0964); 413C (H.M.W. M-1902); 488A (H.M.W. M-2059).

LiteratURE REPORTS: Orłoś 1961, Orłoś \& Twarowska 1967, Domański et al. 1967, Domański 1967, Anonymous 1968, Domański et al. 1973, Bujakiewicz et al. 1992, Grzywacz et al. 1996, Skirgiełło 1997, Orłoś 1960, Niemelä 2013. All literature reports as Phellinus robustus.

REMARKS. In the BF it is rather common, associated with old oaks.

Fomitopsis pinicola (Sw.) P. Karst. Fig. 8A

Specimens EXAMINed: 123A (D.K. 10991); 135C (D.K. 3343B); 194D (D.K.3669C); 225 (D.K. 3687C); 226A (D.K. 3349C); 254D (D.K. 3808E); 255B (D.K. 3653C); 256 (D.K. 3644B); 285A, on Betula pendula fallen trunk, 28 Apr. 2010, Karasiński 5312A (D.K.); 313A, on Picea fallen trunk, 25 July 2009, Karasiński 3509A (D.K.); 314 (D.K. 3303D, DK 3289B); 318 (D.K. 
3880C); 319A (D.K. 3731C); 334C (H.M.W. M-1124); 340 (D.K. 3278E); 342C (D.K. 4784A); 344A (D.K. 3837C); 346D (D.K. 3581B); 370C (D.K. 4731A); 372 (D.K. 3986C); 373C (D.K. 2896); 373D (D.K. 4988A); 373D, on Picea fallen trunk, 13 July 2009, Karasiński 3460C (D.K.); 375D (D.K. 10970); 384B (H.M.W. M-1131); 399A (D.K. 5039A); 412B (H.M.W. M-1934); 412D (H.M.W. M-1329); 429B (H.M.W. M-1125); 463A (H.M.W. M-1285); 537D (H.M.W. M-1099); 572 (Michnówka Reserve), on Alnus glutinosa fallen log, 24 May 2006, Karasiński 060524-5532 (D.K.).

LiteratURE REPORTs: Błoński et al. 1888 (as Polyporus pinicola), Błoński 1889a (as Polyporus pinicola), Siemaszko 1925 (as Polyporus pinicola), Karpiński 1949 (as Fomes marginatus and F. pinicola), Orłoś 1951 (as Fomes marginatus and F. pinicola), Orłoś 1955b (as Fomes marginatus), Nespiak 1956 (as Fomes pinicola), Orłoś 1960 (as Fomes marginatus), Orłoś 1961 (as Fomes marginatus), Dominik 1963 (as Fomes marginatus), Gumińska 1963, Orłoś \& Twarowska 1967, Domański et al. 1967, Domański 1967, Anonymous 1968, Bujakiewicz et al. 1992, Bujakiewicz 1994, Jaroszewicz 1996, Skirgiełło 1997, 1998, Szczepkowski et al. 2008, Bujakiewicz \& Kujawa 2010, Szczepkowski et al. 2010, 2011, Gierczyk et al. 2013, Niemelä 2013, Gierczyk et al. 2014.

REMARKS. This species is very commom in the study area.

\section{Fomitopsis rosea (Alb. \& Schwein.) P. Karst.}

Fig. 8B

SPECimens EXAMINED: 105B (D.K. 3434); 135C (D.K. 3342B), 135D (D.K. 3331A); 194D (D.K. 3665B1); 214B (D.K. 11002) 224D (D.K. 3832A); 225A (D.K. 4128A); 226B (D.K. 3683B); 255D (D.K. 3653B); 256D (D.K. 3652); 257B, on Picea trunk, 21 Sept. 2009, Karasiński 4354B (D.K.); 257D (D.K. 4342B); 260A (D.K. 3370F); 261A (D.K. 3376B); 285A (D.K. 3617A); 287C (D.K. 3778); 288D (D.K. 3738A); 289C (D.K. 3745A); 313B (D.K. 3510); 314C (D.K. 3888B); 315A (D.K. 3894B); 318D (D.K. 3879); 319A (D.K. 3731); 340F (D.K. 3289B); 369, on Picea fallen trunk, 15 Sept. 2005, Karasiński 050915-7807 (D.K.); 369C (D.K. 3490A); 370C (D.K. 3705B); 371C (D.K. 3250A), 373D (D.K. 3458A); 374A (D.K. 3536A1); 375A (D.K. 3577A); 375D (D.K. 10945, D.K. 10975); 398A (H.M.W. M-2717); 399A, on Picea fallen trunk, 1 Nov. 2009, Karasiński 5011B (D.K.); 399B (D.K. 3244B); 402C, on Picea fallen trunk, 22 Sept. 2007, Karasiński 070922-7937 (D.K.); 403A (H.M.W.
M-2796); 412A (H.M.W. M-2711); 414C (H.M.W. M-1005); 415C (H.M.W. M-1472); 463C (H.M.W. M-1291); 476B (H.M.W. M-0970); 487B (H.M.W. M-1137); 488C (Nieznanowo Reserve), on Picea fallen trunk, 13 Sept. 2005, Karasiński 050913-7647 (D.K.); 572 (Michnówka Reserve), on Picea fallen log, 24 May 2006, Karasiński 060524-5606 (D.K.). 697B (Starzyna Reserve), on Picea fallen log, 28 Sept. 2006, Karasiński 060928-6188 (D.K.).

Literature RePORTs: Błoński et al. 1888 (as Polyporus roseus), Błoński 1889a (as Polyporus roseus), Siemaszko 1923 (as Polyporus roseus), Pilát 1950 (as Fomes roseus), Orłoś 1951 (as Fomes roseus), Orłoś $1955 \mathrm{~b}$ (as Fomes roseus), Orłoś 1960 (as Fomes roseus), Orłoś 1961 (as Fomes roseus), Benedix 1967, Domański et al. 1967, Domański 1967, Kotlaba \& Lazebniček 1967, Kreisel 1967, Anonymous 1968, Domański et al. 1973, Skirgiełło 1972, Szczepka 1989, Bujakiewicz et al. 1992, Bujakiewicz 1994, Jaroszewicz 1996, Skirgiełło 1997, Bujakiewicz 2003, Szczepkowski et al. 2008, Bujakiewicz \& Kujawa 2010, Szczepkowski et al. 2011, Gierczyk et al. 2013, Niemelä 2013, Gierczyk et al. 2014.

REMARKS. This is one of the commonest poroid species in the study area, especially in BNP. A single large fallen Picea trunk may often be inhabited by more than 200 basidiomata. Outside the Białowieża Forest it is known in Poland from only a few localities (Wojewoda 2003).

\section{Frantisekia mentschulensis (Pilát) Spirin}

Fig. 8C

SPeCimens eXAmined: 254D, on Fraxinus log covered by mosses, 15 Aug. 2009, Karasiński 3811 (D.K.); 254D, on Acer log, 12 Sept. 2011, Karasiński 6183 (D.K.); 282D, on Populus fallen log, 25 July 2009, Karasiński $3523 B$ (D.K.); 284B, on Fraxinus fallen trunk, 15 Aug. 2009, Karasiński 3815 (D.K.); 319C, on Betula log covered by mosses, 13 Aug. 2009, Karasiński 3753 (D.K.); 340, on fallen trunk of Populus, 28 Aug. 1973, Pouzar (KRAM F-58159 as Tyromyces mentschulensis); 342C, on Carpinus fallen trunk, 17 Oct. 2009, Karasiński 4788 (D.K.); 369C, on Ulmus? fallen trunk, 22 Sept. 2009, Karasiński 4385 (D.K.); 370A, on Populus fallen trunk, 22 Sept. 2009, Karasiński 4402 (D.K.); 370A (D.K. 4403); 370C, on Carpinus betulus fallen trunk, 10 Aug. 2009, Karasiński 3711 (D.K.); 372B, on Populus fallen trunk, 20 Aug. 2009, Karasiński 3970 (D.K.); 372D, on Populus dead standing trunk, 20 Aug. 2009, Karasiński 3971 (D.K.); 372D (D.K. 3985); 399A, 
on Tilia fallen trunk, 1 Nov. 2009, Karasiński 5044 (D.K.); 399, on fallen trunk of Carpinus, 31 Aug. 1973, Holubova s.n. (KRAM F-58160 as Tyromyces mentschulensis); 402, on Populus trunk, 19 Sept. 2013, Karasiński 10087 (D.K.); 402A, on Populus fallen trunk, 19 Sept. 2009, Karasiński 4266 (D.K.).

Literature REPORTs: Kotlaba \& Pouzar 1988 (as Poria fissiliformis), Piątek 2001 (as Antrodiella fissiliformis), Karasiński et al. 2009, Niemelä 2013, Gierczyk et al. 2014 (as Antrodiella fissiliformis).

Remarks. So far, in Poland this species is not known outside the BF. Ryvarden and Gilbertson (1993) reported it from Poland without a precise locality.

\section{Fuscoporia ferruginosa (Schrad.) Murrill}

Fig. 8D

SPECIMENS EXAMINED: 135D, on fallen trunk of deciduous tree, 8 July 2009, Karasiński $3332 a$ (D.K.); 194C, on Populus fallen trunk, 9 July 2009, Karasiński 3364 (D.K.); 334A (H.M.W. M-0046); 344D, on Acer fallen branch, 28 March 2002, Wotkowycki (H.M.W. M-2820); 399B, on Fraxinus excelsior fallen trunk, 2 Nov. 2009, Karasiński 5066 (D.K.); 414C (H.M.W. M-2725).

LiteratURE REPORTS: Pilát 1950 (as Phellinus ferruginosus), Niemelä 2013 (as Phellinus ferruginosus).

\section{Ganoderma applanatum (Pers.) Pat. Fig. 8E}

SPeCimens EXAMINed: 123A (D.K. 10992); 214D (D.K. 11013); 214D (D.K. 11026); 334D, on fallen trunk in Tilio-Carpinetum, 24 March 1993, Wotkowycki (H.M.W. M-1175); 340F, on Acer platanoides fallen trunk, 6 July 2009, Karasiński $3278 C$ (D.K.); 343B, on Acer platanoides fallen log, 16 Aug. 2009, Karasiński 3823 (D.K.); 372C, on Carpinus fallen trunk, 5 July 2009, Karasiński 3252E (D.K.); 375, on Quercus trunk, 19 Sept. 2009, Karasiński 4274 (D.K.); 375D (D.K. 10958); 384C (H.M.W. M-1120); 385C (H.M.W. M-1228); 412B (H.M.W. M-1292); 414B (H.M.W. M-1957); 702B (H.M.W. M-1139).

Literature REPORTs: Błoński et al. 1888 (as Polyporus applanatus), Siemaszko 1925 (as Polyporus ap- planatus), Karpiński 1949 (as Polyporus applanatus), Orłoś 1951 (as Fomes applanatus), Orłoś 1955b, 1960, 1961, Dominik 1963, Orłoś \& Twarowska 1967, Domański et al. 1967, Domański 1967, Nespiak 1968, Skirgiełło 1970, Bujakiewicz et al. 1992, Bujakiewicz 1994, Jaroszewicz 1996, Skirgiełło 1997, 1998 (as Ganoderma lipsiense), Sokół 2000, Szczepkowski et al. 2008, Bujakiewicz \& Kujawa 2010, Szczepkowski et al. 2011, Gierczyk et al. 2013, Niemelä 2013, Gierczyk et al. 2014.

REMARKS. This is common species in the study area but was underrecorded during recent fieldwork.

Ganoderma lucidum (Curtis) P. Karst. Fig. 8F

Specimens exAmined: 194C, at base of living Quercus, 9 July 2009, Karasiński 3357 (D.K.); 257D, among mosses at base of Quercus old living tree, 21 Sept. 2009, Karasiński 4364 (D.K.).

Literature REPORTS: Dominik 1963, Hołownia 1974, Bujakiewicz et al. 1992, Skirgiełło 1997, Sokół 2000, Niemelä 2013.

REMARKS. This species is rare in the study area.

\section{Gelatoporia subvermispora (Pilát) Niemelä}

Fig. $8 \mathrm{G}$

Specimens EXAMINED: 224B, on Pinus fallen log, 19 Aug. 2009, Karasiński 3944 (D.K.); 256A, on Picea fallen trunk, 20 July 2009, Karasiński 3652 (D.K.); 282D, on Picea fallen trunk, 25 July 2009, Karasiński 3535 (D.K.); 284B, on Alnus fallen log, 15 Aug. 2009, Karasiński 3816 (D.K.); 313B, on Picea fallen log, 25 July 2009, Karasiński 3525 (D.K.); 340F, on Picea fallen trunk, 6 July 2009, Karasiński 3294 (D.K.); 344B, on Quercus fallen log, 16 Aug. 2009, Karasiński 3830 (D.K.); 374C, on Pinus fallen log, 21 Aug. 2009, Karasiński 4004 (D.K.); 375B, on Picea rotten log, 27 July 2009, Karasiński 3579 (D.K.); 398G, on Picea fallen trunk, 24 July 2009, Karasiński 3504A (D.K.).

Literature REPORTs: Domański 1967 (as Poria subvermispora), Domański 1969d (as Fibuloporia subvermispora), Domański 1972b (as Fibuloporia subvermispora), Niemelä 1985, Niemelä 2013.

Fig. 8. A - Fomitopsis pinicola (Sw.) P. Karst., B - Fomitopsis rosea (Alb. \& Schwein.) P. Karst., C - Frantisekia mentschulensis (Pilát) Spirin, D - Fuscoporia ferruginosa (Schrad.) Murrill, E - Ganoderma applanatum(Pers.) Pat., F - Ganoderma lucidum (Curtis) P. Karst., G - Gelatoporia subvermispora (Pilát) Niemelä, H - Gloeophyllum odoratum (Wulfen) Imazeki. Photo D. Karasiński (A-H). 

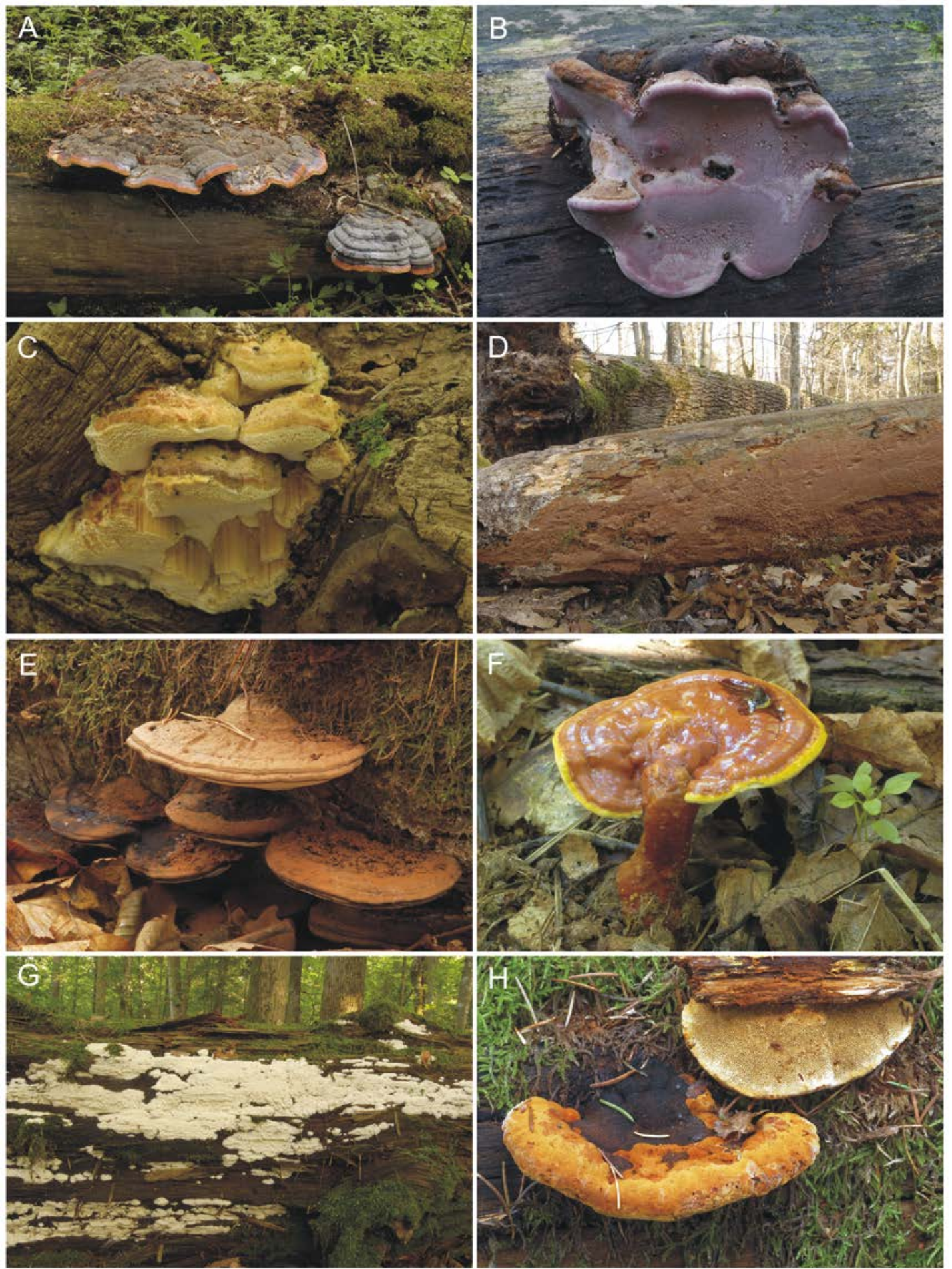
REMARKS. This species appears to be not rare in the study area. In Poland it was reported from BNP and recently from Kampinos National Park in central Poland (Karasiński et al. 2015), and also found in the Lipówka Reserve in the Niepołomice Forest in the south of the country (D. Karasiński, unpubl.).

\section{Gloeophyllum abietinum (Bull.) P. Karst.}

Specimens eXamined: 384C (Sacharewo), on Picea abies fallen trunk, 10 March 1993, Wotkowycki (H.M.W. M-1143); BNP, Wilczy Szlak, on Picea thick decorticate trunk in windthrow area, 14 Sept. 2009, Niemelä 8653 (KRA).

Literature RePORTs: Błoński 1889a, Domański 1967, Anonymous 1968, Niemelä 2013.

\section{Gloeophyllum odoratum (Wulfen) Imazeki}

Fig. $8 \mathrm{H}$

SPECIMENS EXAMINED: 285, on Picea fallen trunk, 28 Apr. 2009, Karasiński 5334A (D.K.); 374D (D.K. 10943); 375D (D.K. 10963); 398D (H.M.W. M-1361); 414A (H.M.W. M-1920); 488C (Nieznanowo Reserve), on Picea fallen log, 13 Sept. 2005, Karasińśki 050913-7629 (D.K.); 601A (H.M.W. M-1105); 702B (H.M.W. M-1108); BNP, SE part, west of Dziedzinka house, on Picea falen trunk, 12 Oct. 2008, Niemelä 8458 (KRA).

LiTERATURE REPORTS: Błoński et al. 1888 (as Trametes odorata), Błoński 1889a (as Ochroporus odoratus), Siemaszko 1923 (as Trametes odotata), Orłoś 1960 (as Anisomyces odoratus), Orłoś 1961 (as Anisomyces odoratus), Domański et al. 1967 (as Osmoporus odoratus), Domański 1967 (as Osmoporus odoratus), Nespiak 1968, Bujakiewicz et al. 1992, Jaroszewicz 1996, Skirgiełło 1997, Szczepkowski et al. 2008, Bujakiewicz \& Kujawa 2010, Szczepkowski et al. 2010, Szczepkowski et al. 2011, Gierczyk et al. 2013, Niemelä 2013, Gierczyk et al. 2014.

REMARKS. This species is common in the study area.

\section{Gloeophyllum sepiarium (Wulfen) P. Karst.}

Specimens examined: 182D (D.K. 10995); 335D, on Picea fallen trunk, 24 March 1993, Wolkowycki (H.M.W. M-1126); 335C (H.M.W. M-1123); 369A, on Picea fallen trunk, 14 July 2009, Karasiński 3488B (D.K.); 384A (H.M.W. M-1487); 412B (H.M.W. M-1488); 413A (H.M.W. M-1142); 415C (H.M.W. M-1471); 464D (H.M.W. M-1127); 488C (H.M.W. M-0972); 537D (H.M.W. M-1111).

LITERATURE REPORTS: Błoński et al. 1888 (as Lenzites sepiaria), Błoński 1889a (as Lenzites sepiaria), Siemaszko 1925 (as Lenzites sepiaria), Karpiński 1949 (as Lenzites sepiaria), Domański 1967, Nespiak 1968, Bujakiewicz et al. 1992, Jaroszewicz 1996, Skirgiełło 1997, Orłoś 1960, Niemelä 2013, Gierczyk et al. 2013, 2014.

Remarks. In Poland it is a common species in managed coniferous forests, but rather uncommon in the study area.

Gloeoporus dichrous (Fr.) Bres.

Fig. 9A

Specimens examined: 402A, on Populus fallen trunk, 31 Oct. 2009, Karasiński 4979 (D.K.).

LITERATURE REPORTS: Domański 1967, Domański 1970c (as Polyporus dichrous), Domański et al. 1973, Gierczyk et al. 2013, Niemelä 2013.

REMARKs. This species appears to be rare in the study area.

Gloeoporus pannocinctus (Romell) J. Erikss.

Fig. 9B

Specimens examined: 135C, on Betula pendula fallen trunk, 8 July 2009, Karasiński 3342d (D.K.); 214D (D.K. 11004); 224D (D.K. 3932D); 225A (D.K. 4130), 225B (D.K. 4600); 226A (D.K. 3349); 256D (D.K. 3769A); 258C (D.K. 4357); 261A (D.K. 3392); 284B (D.K. 3814D); 313A (D.K. 3507); 315D (D.K. 3928); 319C (D.K. 3748B); 343B (D.K. 3841B); 370C (3707B); 370D (D.K. 4399); 372D, on Populus fallen trunk, 20 Aug. 2009, Karasiński 3967 (D.K.); 372D (D.K. 3975A); 372D (D.K. 3982B); 373C, on Populus fallen branch, 20 Aug. 2009, Karasiński 3975b (D.K.);

Fig. 9. A - Gloeoporus dichrous (Fr.) Bres., B - Gloeoporus pannocinctus (Romell) J. Erikss., C - Gloeoporus taxicola (Pers.) Gilb. \& Ryvarden, D - Grifola frondosa (Dicks.) Gray, E - Hapalopilus rutilans (Pers.) Murrill, F - Hapalopilus ochraceolateritius (Bondartzev) Bondartzev \& Singer (specimen photographed in Kampinos National Park, central Poland), G - Heterobasidion parviporum Niemelä \& Korhonen, H - Inocutis dryophila (Berk.) Fiasson \& Niemelä. Photo D. Karasiński (A-H). 

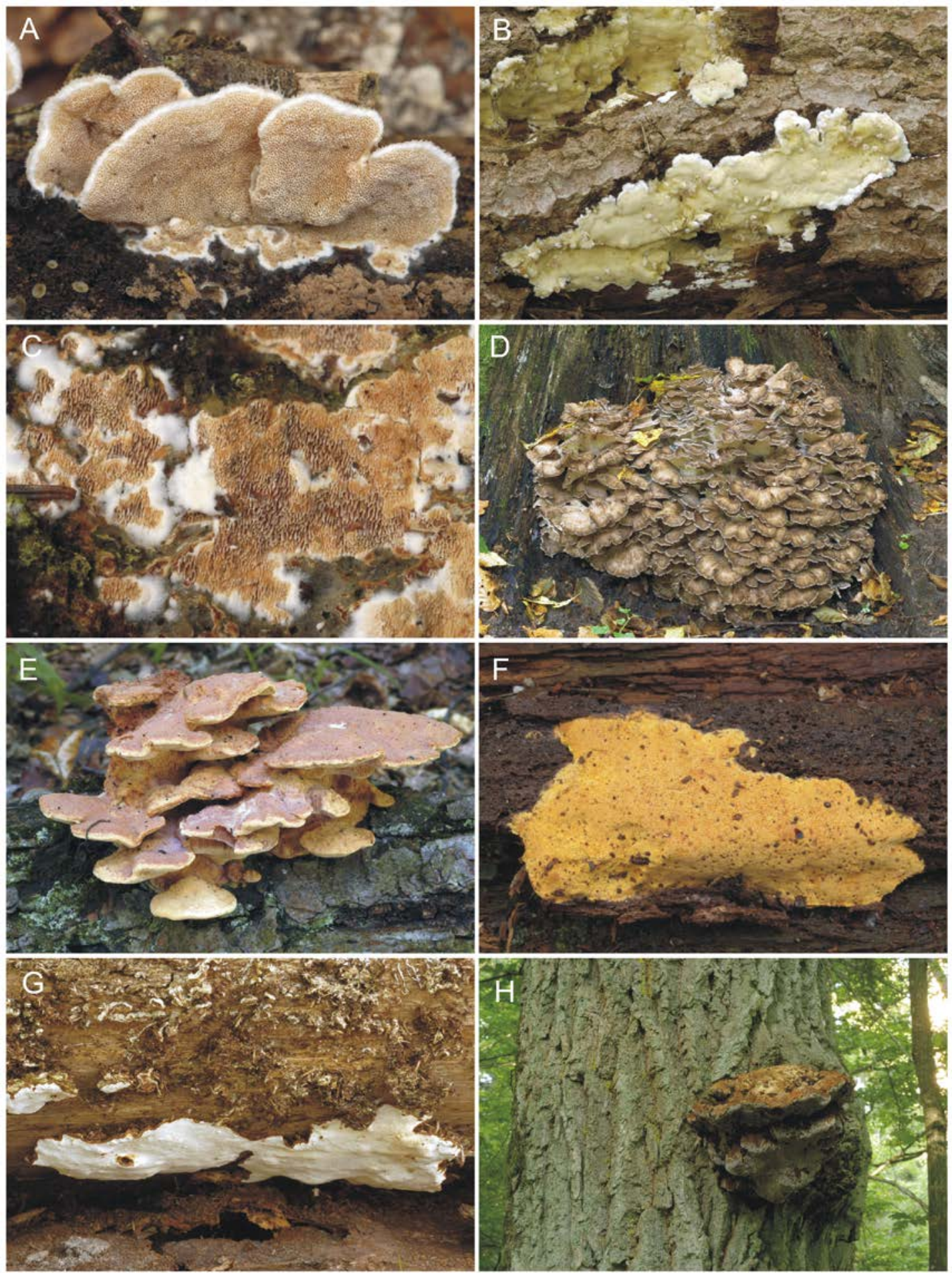
374B (D.K. 4186), 375A (D.K. 4286); 398 (D.K. 3504) 402A (D.K. 3533); 443A (W. Szafer Landscape Reserve), on fallen trunk, 23 June 2008, Karasiński 1698 (D.K.).

LiterATURE REPORTS: Domański 1965, 1967, Anonymous 1968, Domański 1970c (as Polyporus pannocinctus), Domański 1972b, Szczepkowski et al. 2011, Niemelä 2013, Gierczyk et al. 2014 (as Gelatoporia pannocincta).

REMARKS. In the BF it is a rather common species on dead hardwoods, especially Populus and Betula.

\section{Gloeoporus taxicola (Pers.) Gilb. \& Ryvarden}

Fig. 9C

Specimens eXAmined: 284D, on Pinus decorticate trunk, 7 July 2009, Karasiński 3315 (D.K.); 316C, on Pinus fallen branch, 27 Apr. 2010, Karasiński 5273A (D.K.); 316D, on bark of Pinus fallen trunk, 1 May 2010, Karasiński 5357 (D.K.); 399C (H.M.W. M-2800); 412B (H.M.W. M-2705).

LiTERATURE REPORTS: Domański 1967 (as Meruliopsis taxicola), Niemelä 2013 (as Meruliopsis taxicola).

REMARKs. This species possibly is not rare but undercollected in recent studies.

\section{Grifola frondosa (Dicks.) Gray}

Fig. 9D

SPECIMENS EXAMINED: 257D, at base of very old Quercus dead standing tree, 21 Sept. 2009, Karasiński 4365 (D.K.); $\mathbf{2 8 5 C}$, at base of Quercus living tree, 13 Sept. 2011, Karasiński 6281 (D.K.); 290, at base of Quercus living tree, 22 Sept. 2011, Karasiński 6477 (D.K.); 314A, at base of Quercus dead standing tree, 13 Sept. 2011, Karasiński 6238 (D.K.); 370D, at base of Quercus dead standing trunk, 15 Oct. 2009, Karasiński 4751 (D.K.); 418B (H.M.W. M-0005).

LiterATURE REPORTS: Orłoś 1955b, Domański et al. 1967, 1973, Hołownia 1974, Szczepka \& Sokół 1991, Bujakiewicz et al. 1992, Skirgiełło 1997, Szczepkowski \& Piętka 2008, Szczepkowski et al. 2011, Niemelä 2013.

\section{Hapalopilus ochraceolateritius (Bondartzev) Bondartzev \& Singer \\ Fig. 9F}

Specimens examined: BF, on Pinus fallen trunk, 17 July 1958, Domański (KRAM F-SD 716); BF, on Pinus trunk, Oct. 1955, Domański (KRAM F-SD 814); BF, on Picea fallen trunk, 20 Oct. 1963, Domański (KRAM F-SD 3424).
LiTERATURE REPORTs: Domański 1965, 1967, 1972b, Niemelä 2013.

REMARKS. The specimens cited above are preserved in KRAM F-SD and possibly are the same as in literature reports. Hapalopilus ochraceolateritius seems to be a true rarity in the study area; since 1963 it has not been recollected, including during recent intensive fieldwork. According to available data, the basidiomata appear from July to October. Morphologically similar $H$. aurantiacus differs by having larger pores (1-3 per mm vs. 3-6 per mm in H. ochraceolateritius) and larger (mostly wider) basidiospores $(5.2-6.4 \times 2.2-2.8 \mu \mathrm{m}$ vs. $4.2-5.4$ $\times 1.8-2.2 \mu \mathrm{m}$ in H. ochraceolateritius).

Hapalopilus rutilans (Pers.) Murrill Fig. 9E

SPeCimens EXAmined: 194C, on dead standing trunk of Quercus, 9 July 2009, Karasiński 3356b (D.K.); 356A (H.M.W. M-1115); 413A (H.M.W. M-1128); 414B (H.M.W. M-1953); 414D (H.M.W. M-2196); 444B, on Corylus avellana, 22 Aug. 2004, Wotkowycki (H.M.W. M-3431); 463A (H.M.W. M-1744).

LITERATURE REPORTS: Błoński 1889a (as Ochroporus nidulans), Domański et al. 1967 (as Hapalopilus nidulans), Domański 1967 (as Hapalopilus nidulans), Anonymous 1968 (as Hapalopilus nidulans), Domański et al. 1973 (as Hapalopilus nidulans), Bujakiewicz et al. 1992, Skirgiełło 1997, Bujakiewicz \& Kujawa 2010 (as Hapalopilus nidulans), Niemelä 2013, Gierczyk et al. 2014 (as Hapalopilus nidulans).

REMARKS. This species is not common but possibly also undercollected in recent studies.

Heterobasidion annosum (Fr.) Bref.

Literature RePOrts: Błoński et al. 1888 (as Polyporus annosus), Karpiński 1949 (as Fomes annosus), Orłoś 1951 (as Trametes radiciperda), Orłoś 1955b, 1960, 1961 (as Fomes annosus), Orłoś \& Twarowska 1967, Domański et al. 1967, Domański 1967, Domański et al. 1973 (as Heterobasidion annosus f. macraulos), Skirgiełło 1976, Bujakiewicz et al. 1992, Jaroszewicz 1996, Grzywacz et al. 1996, Skirgiełło 1997, Łakomy et al. 2000, Szczepkowski et al. 2008, Bujakiewicz \& Kujawa 2010, Gierczyk et al. 2013, Niemelä 2013, Gierczyk et al. 2014.

REMARKS. Literature reports published before 2000 refer to the species sensu lato. In KRAM F-SD all specimens collected by Domański in the 
study area belong to Heterobasidion parviporum Niemelä \& Korhonen (see below). We did not collect Heterobasidion annosum s.str. during recent fieldwork. Recent records referring to the species sensu stricto were published by Szczepkowski et al. (2008), Bujakiewicz and Kujawa (2010), Gierczyk et al. (2013, 2014) and Niemelä (2013).

Heterobasidion parviporum Niemelä \& Korhonen

Fig. 9G

Specimens eXamined: 285C, on Picea stump, 21 Sept. 2010, Karasiński 5952 (D.K.); 340G, on Picea fallen trunk, 16 Sept. 2009, Karasiński 4216B (D.K.); 402A, on Picea rotten stump, 22 Apr. 2009, Karasiński 2855 (D.K.); 402A, on Picea fallen trunk, 25 June 2008, Karasiński 1735 (D.K.); 402B, on Picea fallen trunk, 15 July 2009, Wotkowycki (H.M.W. M-3653).

LITERATURE REPORTS: Kowalski \& Łakomy 1998 (as Heterobasidion annosum intersterility group S), Łakomy et al. 2000 (as Heterobasidion annosum intersterility group S), Niemelä 2013, Gierczyk et al. 2013, 2014.

\section{Inocutis dryophila (Berk.) Fiasson \& Niemelä}

Fig. $9 \mathrm{H}$

SPECIMENS EXAMINED: 257C, on trunk of Quercus living tree, 21 Sept. 2009, Karasiński 4337 (D.K.); 318D, on trunk of Quercus old living tree, 17 Aug. 2009, Karasiński 3883A (D.K.); 375B, on trunk of Quercus living tree, 27 July 2009, Karasiński 3592 (D.K.); 402A, on trunk of Quercus living tree $c a 100$ years old, 31 Oct. 2009, Karasiński 4978 (D.K.); 416A (H.M.W. M-3656); 442 (W. Szafer Landscape Reserve), on Quercus robur trunk, 23 June 2008, Karasiński 1702 (D.K.).

LiTERATURE REPORTS: Karpiński 1949 (as Inonotus dryophilus), Domański et al. 1967, Domański et al. 1973 (as Inonotus dryophilus), Komorowska 1983 (as Inonotus dryophilus), Niemelä 2013 (as Inonotus dryophilus).

\section{Inocutis rheades (Pers.) Fiasson \& Niemelä}

Fig. 10A

LITERATURE REPORTS: Domański et al. 1967 (as Inonotus rheades), Skirgiełł 1998 (as Inonotus rheades), Niemelä 2013 (as Inonotus rheades).

REMARKS. We did not confirm this species in recent studies, and it was not found among the specimens deposited in KRAM F-SD. According to Niemelä (2013) it is common in the study area.
Inonotus cuticularis (Bull.) P. Karst. Fig. 10B

Specimens examined: BF, Palace Park in Białowieża, on deciduous stump, Oct. 1955, Domański (KRAM F-SD 602).

LiTERATURE REPORTS: Domański et al. 1967, 1973, Niemelä 2013.

REMARKS. This species appears to be rare and was not confirmed by recent observations. Only one specimen (cited above) collected in 1955 is deposited in KRAM F-SD.

Inonotus dryadeus (Pers.) Murrill Fig. 10C

Literature REPORTS: Karpiński 1949 (as Polyporus dryadeus), Orłoś 1955b, Domański et al. 1967, Domański et al. 1973 (as Inonotus dryadeus), Bujakiewicz 2003, Bujakiewicz \& Kujawa 2010, Niemelä 2013.

REMARKS. We did not confirm this species in recent work and didn not find it among the specimens stored in KRAM F-SD, but it was collected and recently published from the study area by Bujakiewicz (2003) and Bujakiewicz and $\mathrm{Ku}-$ jawa (2010). Niemelä (2013) reported it based on literature data (after Domański 1972b).

Inonotus hispidus (Bull.) P. Karst. Fig. 10D

Specimens examined: 413A (Sacharewo), on trunk of Malus domestica living tree, 30 July 2000, Wolkowycki (H.M.W. M-1919).

\section{LITERATURE REPORTS: Niemelä 2013.}

REMARKS. Inonotus hispidus is characterized by having usually large, sessile, semicircular basidiomata with a strongly hispid upper surface and ovoid basidiospores 9-12 × 6-9 $\mu \mathrm{m}$, the largest among European species of Inonotus. In Poland almost all reports are of it growing synanthropically in places such as orchards, gardens, parks and avenues (Piątek 2000). Similarly, in the BF it was collected only once in an old orchard in the vicinity of Hajnówka (Sacharewo). Niemelä (2013) listed this species as 'Rather common in Central Europe', and apparently did not find it in the BF.

Inonotus obliquus (Pers.) Pilat

Fig. 10E

Specimens eXAmined: 316, on Betula pubescens fallen trunk, 27 Apr. 2010, Karasiński 5279 (D.K.); 
319C, on Betula fallen log, 13 Aug. 2009, Karasiński $3748 G$ (D.K.); 340G, on Betula dead standing trunk, 16 Sept. 2009, Karasiński 4228 (D.K.); 442 (W. Szafer Landscape Reserve), on Betula fallen trunk, 23 June 2008, Karasiński 1705 (D.K.).

Literature RePORTS: Błoński et al. 1888 (as Polyporus obliquus), Błoński 1889a (as Ochroporus obliquus), Pilát 1950, Orłoś 1951 (as Fomes nigricans), Mańka \& Stube 1952 (as Poria obliqua), Orłoś 1961, Domański 1965, 1967, Anonymous 1968, Domański 1972b.

\section{Irpex lacteus (Fr.) Fr.}

Fig. 10F

Specimens eXAmined: 402, on fallen branches of Betula, 19 Sept. 2013, Karasiński 10088 (D.K.).

LITERATURE REPORTS: Błoński 1889a (as Sistotrema canesces), Domański 1967, Niemelä 2013.

REMARKs. This species appears to be rare in the study area.

\section{Ischnoderma benzoinum (Wahlenb.) P. Karst.}

Fig. 10G

Specimens eXAmined: 226A, on Picea fallen trunk, 30 July 2009, Karasiński $3687 G$ (D.K.); 226A, at base of Picea dead standing trunk, 20 Sept. 2011, Karasiński 6390 (D.K.); 253C (D.K. 4091A); 260D (D.K. 4612); 282D, on Picea fallen trunk, 25 July 2009, Karasiński 3519 (D.K.); 284B, at base of Picea stump, 15 Aug. 2009, Karasiński 3821 (D.K.); 284D, on Pinus fallen log, 13 Sept. 2011, Karasiński 6275 (D.K.); 314C, on Picea fallen log, 25 Apr. 2010, Karasiński 5167 (D.K.); 315B (D.K. 3913); 370C, on Picea fallen trunk, 10 Aug. 2009, Karasiński 3701A (D.K.); 374C, on Pinus fallen trunk, 21 Aug. 2009, Karasiński 4005 (D.K.); 375A (D.K. 4282); 375D (D.K. 10968); 439A (H.M.W. M-1011); 463B (H.M.W. M-1492); 485C (H.M.W. M-1133); 487B (H.M.W. M-1206).

LitERATURE REPORTS: Bujakiewicz et al. 1992, Jaroszewicz 1996, Bujakiewicz \& Kujawa 2010, Szczepkowski et al. 2011, Niemelä 2013, Gierczyk et al. 2014.

REMARKS. In the study area the species occurs mostly on Picea and very rarely on Pinus. On the latter substrate the basidiomata are smaller and usually grow solitary.

Ischnoderma resinosum (Fr.) P. Karst. Fig. 10H

Specimens eXamined: 257D, on Tilia fallen log, 21 Sept. 2009, Karasiński 4366 (D.K.); 341D, on Acer platanoides fallen trunk, 17 Oct.2009, Karasiński 4765 (D.K.); BNP, on Carpinus thick fallen trunk, mosscovered but still hard wood, 11 Oct. 2008, Niemelä 8437 (KRA).

Literature REPORTS: Orłoś 1960, Domański et al. 1967, Domański 1967, Kreisel 1967, Bujakiewicz et al. 1992, Skirgiełło 1997, Niemelä 2013.

REMARKS. In the BF this species appears to be much rarer than Ischnoderma benzoinum.

Junghuhnia collabens (Fr.) Ryvarden Fig. 11A

Specimens eXamined: 253C (D.K. 4091B); 254D (D.K. 3800D); 256D, on well rotted Picea fallen trunk, 14 Aug. 2009, Karasiński 3771 (D.K.); 282D, on Picea fallen trunk, 25 July 2009, Karasiński 3521 (D.K.); 315A (D.K. 3901); 319C, on Picea fallen log, 13 Aug. 2009, Karasiński 3754C (D.K.); 342C (D.K. 4787); 369A, on Picea fallen trunk, 14 July 2009, Karasiński 3489 (D.K.); 374D (D.K. 4195); 374B (D.K. 4659); 399B (D.K. 5056); 697B (Starzyna reserve), on Picea fallen log, 28 Sept. 2006, Karasiński 060928-6197 (D.K.).

LiterATURE REPORTS: Domański 1965, 1967 (as Chaetoporus rixosus), Kotlaba \& Lazebniček 1967 (as Chaetoporus rixosus), Anonymous 1968 (as Chaetoporus rixosus), Domański 1972b (as Chaetoporus collabens), Szczepkowski et al. 2008, Niemelä 2013, Gierczyk et al. 2014.

Junghuhnia fimbriatella (Peck) Ryvarden

Fig. 11B

SPeCimens EXAMINED: 256D, on Acer platanoides fallen $\log$ close to living Ganoderma applanatum basidiomata, 14 Aug. 2009, Karasiński 3770 (D.K.); 343B, on Acer platanoides fallen log together with Ganoderma applanatum, 16 Aug. 2009, Karasiński

Fig. 10. A - Inocutis rheades (Pers.) Fiasson \& Niemelä (specimen photographed in Kaszuby Landscape Park, N Poland), B - Inonotus cuticularis (Bull.) P. Karst. (specimen photographed in Cracow, S Poland), C - Inonotus dryadeus (Pers.) Murrill (specimen photographed in Puszcza Niepołomicka Forest, S Poland), D - Inonotus hispidus (Bull.) P. Karst., E - Inonotus obliquus (Pers.) Pilat, F - Irpex lacteus (Fr.) Fr., G - Ischnoderma benzoinum (Wahlenb.) P. Karst., H - Ischnoderma resinosum (Fr.) P. Karst. Photo D. Karasiński (A-H). 

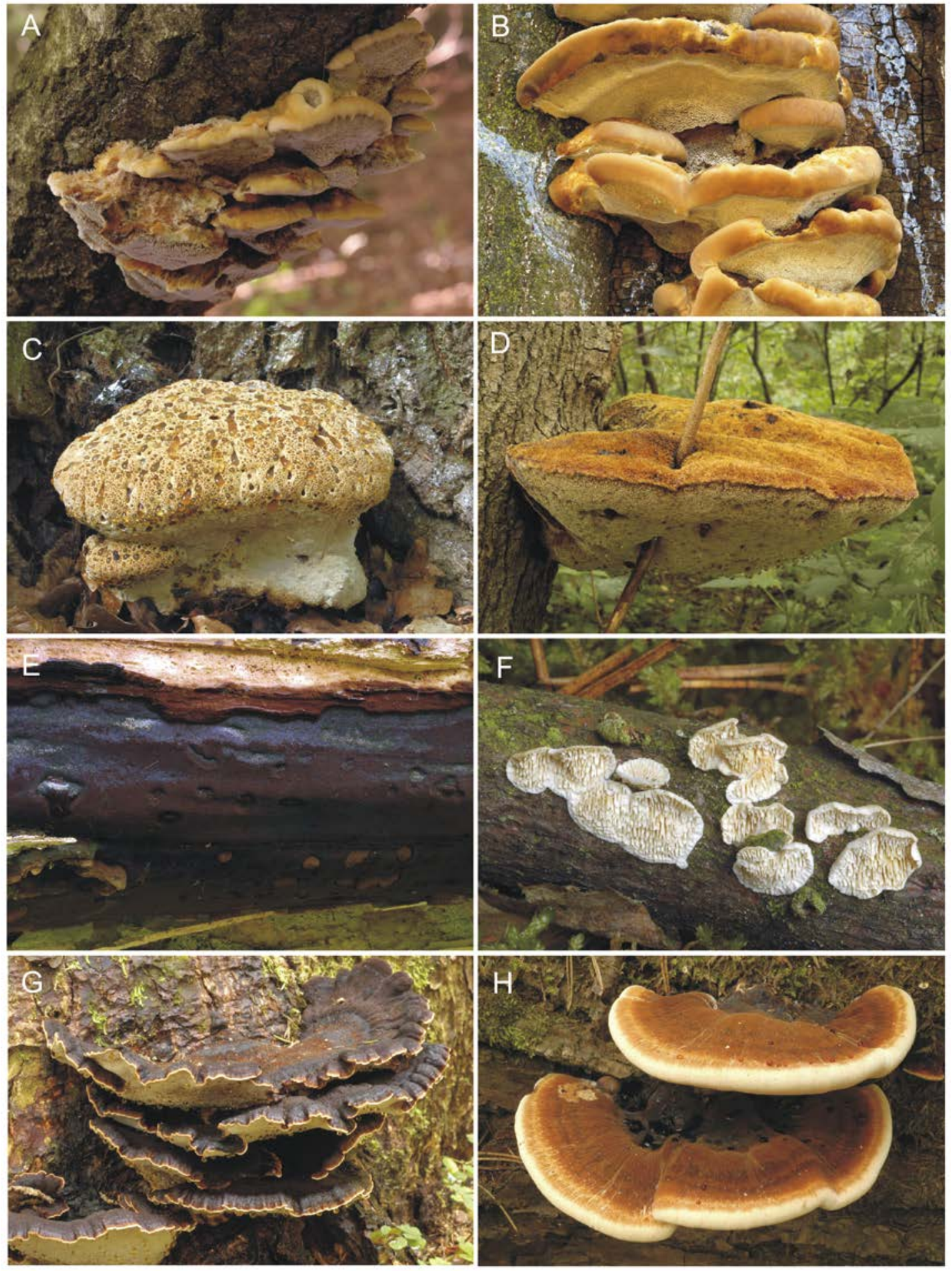
3823 (D.K.); 374A, on Quercus fallen trunk together with Ganoderma applanatum, 19 Sept. 2009, Karasiński 4275 (D.K.).

LiterATURE REPORTS: None. The species is new for Poland.

REMARKS. The species is characterized by having resupinate, cream-colored basidiomata with pores 3-5 per $\mathrm{mm}$, a strongly rhizomorphic margin, large, encrusted skeletocystidia with subhymenial or tramal origin and small, broadly ellipsoid to subglobose basidiospores 2.5-3.5 $\times$ 1.8-2.5 $\mu \mathrm{m}$ (in specimens examined). Junghuhnia fimbriatella seems to be a very rare successor of the common Ganoderma applanatum. This association, mentioned earlier by other authors (e.g., Ryvarden \& Gilbertson 1993), was confirmed in all recent records of the species in the BF. Ryvarden and Gilbertson (1993) and Ryvarden and Melo (2014) reported Junghuhnia fimbriatella from Poland based on Große-Brauckmann and Nuss (1991). However, in the latter publication there are no data on the occurrence of this species in Poland. Hence it is reported here as a new national record.

\section{Junghuhnia lacera (P. Karst.) Niemelä}

\& Kinnunen

Fig. 11C

SPECIMENS EXAMINED: 318D, on bark of Populus fallen trunk, 17 Aug. 2009, Karasiński 3876 (D.K.); 375B, on Quercus fallen branch, 13 Oct. 2009, Karasiński 4665 (D.K.).

LiTERATURE REPORTS: Domański 1972b (as Cheatoporus separabilimus), Niemelä 2013.

REMARKS. This species appears to be rare in the study area.

Junghuhnia luteoalba (P. Karst.) Ryvarden

Fig. 11D

Specimens eXamined: 260A, on Pinus fallen trunk, 12 Oct. 2009, Karasiński 4645 (D.K.); 260C, on Pinus fallen log, 12 Oct. 2009, Karasiński 4605 (D.K.); 284A, on Pinus trunk, 13 Sept. 2011, Karasiński 6280 (D.K.);
374C, on Pinus fallen log, 15 Sept. 2009, Karasiński 4164 (D.K.); 400B, on Picea fallen trunk, 5 July 2009, Karasiński 3263 (D.K.); BF, on Picea trunk, 11 Aug. 1962, Domański (KRAM F-SD as Tyromyces semisupinus).

LiTERATURE REPORTS: Domański 1965, 1967 (as Chaetoporus variecolor), Domański 1972b (as Chaetoporus luteoalbus), Niemelä 2013.

Junghuhnia nitida (Pers.) Ryvarden Fig. 11E

Specimens eXAmined: 260D, on Betula fallen branch, 12 Oct. 2009, Karasiński 4632 (D.K.); 318D, on Populus fallen trunk, 10 March 2002, Wotkowycki (H.M.W. M-2907); 399D, on Populus fallen branch, 2 Nov. 2009, Karasiński 5050 (D.K.); 402A, on Populus fallen branch, 31 Oct. 2009, Karasiński 4969B (D.K.); 417 (W. Szafer Landscape Reserve), on Populus fallen trunk, 29 Sept. 2006, Karasiński 060929-6337 (D.K.); 438C (H.M.W. M-1271).

Literature REPORTS: Błoński 1889a (as Polyporus micans), Domański 1965, 1967 (as Chaetoporus euporus), Domański 1972b (as Chaetoporus nitidus), Niemelä 2013.

REMARKS. It is close to Junghuhnia lacera but differs in having smaller and more regular pores 5-7 per mm (2-5 per $\mathrm{mm}$ in J. lacera), a nonrhizomorphic margin and slightly smaller basidiospores.

Laetiporus sulphureus (Bull.) Murrill Fig. 11F

SPeCimens eXamined: 214D (Szczekotowo Reserve), on Quercus big fallen log, 17 Oct. 2014, Karasiński 11010 (D.K.); 402C, at base of Quercus dead standing trunk, 22 Sept. 2011, Karasiński 6445A (D.K.); BNP, SE part, west of Dziedzinka house, at base of big Quercus robur, 12 Oct. 2008, Niemelä 8460 (KRA); BF, on Quercus living tree, 12 June 1959, Domański (KRAM F-SD 398 as Grifola sulphurea).

Literature RePorts: Błoński et al. 1888 (as Polyporus rostafińskii nov. sp.), Błoński 1889b (as Polyporus Rostafińskii), Siemaszko 1925 (as Polyporus sulphureus), Karpiński 1949 (as Polyporus sulphureus), Orłoś 1951, 1955b (as Polyporus sulphureus), Orłoś 1960, 1961 (as Grifola sulphurea), Domański et al.

Fig. 11. A - Junghuhnia collabens (Fr.) Ryvarden, B - Junghuhnia fimbriatella (Peck) Ryvarden, C - Junghuhnia lacera (P. Karst.) Niemelä \& Kinnunen, D - Junghuhnia luteoalba (P. Karst.) Ryvarden, E - Junghuhnia nitida (Pers.) Ryvarden, F - Laetiporus sulphureus (Bull.) Murrill, G - Lenzites betulinus (L.) Fr., H - Leptoporus mollis (Pers.) Quél. Photo D. Karasiński (A-H). 

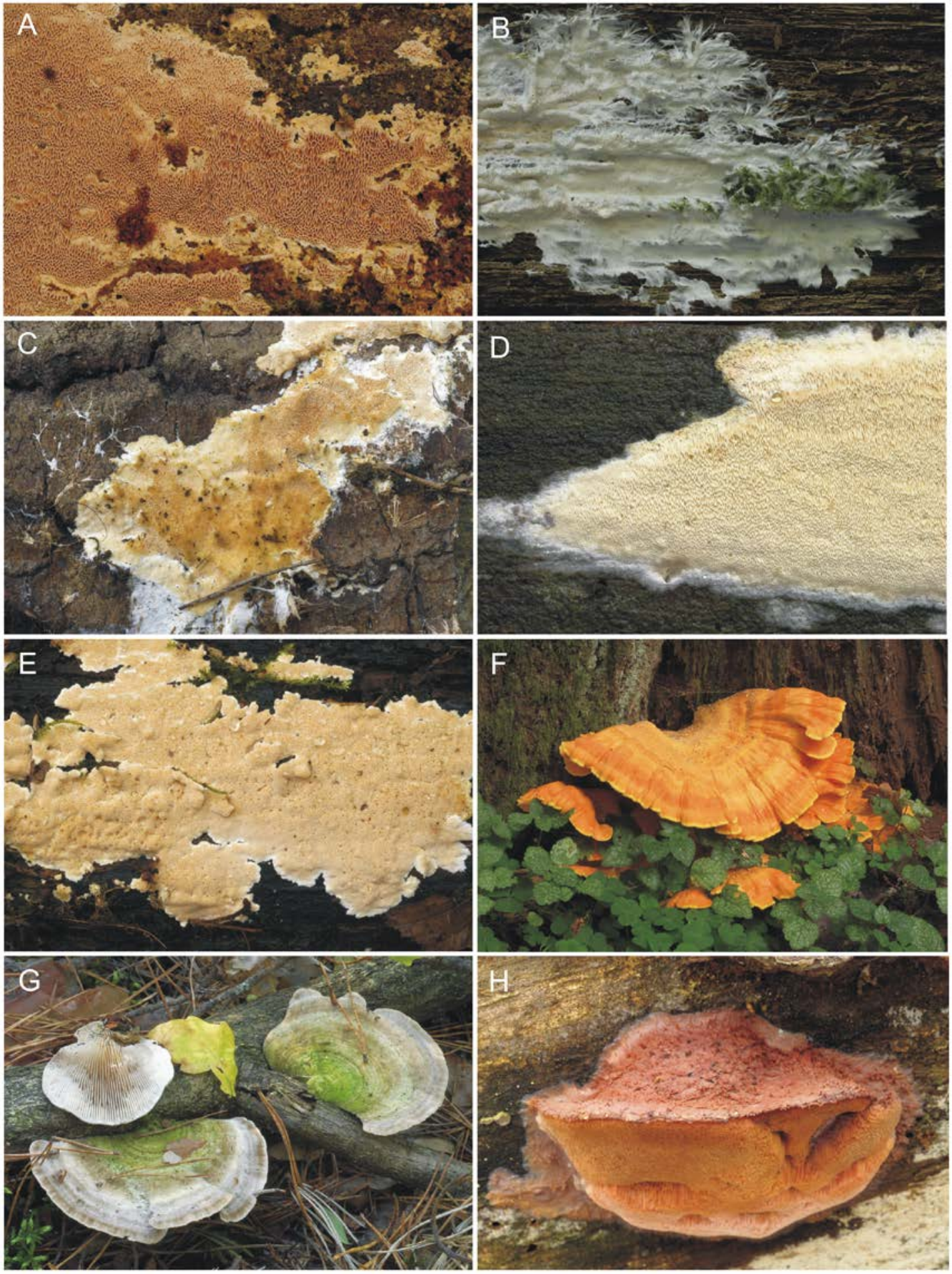
1967, Domański 1967, Anonymous 1968, Nespiak 1968, 1970, Bujakiewicz et al. 1992, Jaroszewicz 1993, 1996, Skirgiełło 1997, Szczepkowski et al. 2008, Bujakiewicz \& Kujawa 2010, Niemelä 2013, Gierczyk et al. 2014.

REMARKs. This species is very common in Poland, but based on field observations is moderately common in the study area, mainly on old Quercus and rarely on Salix.

\section{Lenzites betulinus (L.) Fr.}

Fig. $11 \mathrm{G}$

Specimens examined: 370C, on Tilia fallen trunk, 10 Aug. 2009, Karasiński 3707 (D.K.); 372D, on Populus fallen log, 20 Aug. 2009, Karasiński 3993 (D.K.); 413C (H.M.W. M-2153); 571B, on Betula pendula fallen trunk, 27 Aug. 1990, Wolkowycki (H.M.W. M-1110); 632D (H.M.W. M-1112); BF, on deciduous trunk, 18 Sept. 1960, Domański (KRAM F-SD 1352).

LITERATURE REPORTS: Błoński et al. 1888 (as Lenzites betulina), Błoński 1889a (as Lenzites flaccida), Orłoś 1961 (as Trametes betulina), Domański et al. 1973 (as Lenzites betulina f. flaccida), Bujakiewicz et al. 1992, Skirgiełło 1997, Gierczyk et al. 2013, Niemelä 2013, Gierczyk et al. 2014.

Leptoporus mollis (Pers.) Quél.

Fig. $11 \mathrm{H}$

Specimens EXAmined: 135D, on Picea fallen trunk, 8 July 2009, Karasiński 3335 (D.K.); 224B, on Picea trunk, 19 Aug. 2009, Karasiński 3939a (D.K.); 224B (D.K. 3952), 285A (D.K. 3956); 286D, on Picea fallen trunk, 14 Aug. 2009, Karasiński 3776 (D.K.); 319C, on Picea fallen trunk, 28 July 2009, Karasiński 3610 (D.K.); 374C, on Pinus fallen log, 26 July 2009, Karasiński 3551 (D.K.); 374D (D.K. 3960, 4006, 4163); 375D, on Pinus log, 24 Sept. 2010, Karasiński 6051 (D.K.); 402A (D.K. 4980).

LITERATURE REPORTS: Domański et al. 1967 (as Tyromyces mollis), Domański 1967 (as Tyromyces mollis), Domański et al.1973 (as Tyromyces mollis), Bujakiewicz et al. 1992 (as Tyromyces mollis), Skirgiełło 1997 (as Tyromyces mollis), Niemelä 2013.

REMARKS. This species is not common in the study area. In Poland its largest known population was recently found in Kampinos National Park (Karasiński et al. 2015).

\section{Lindtneria flava Parmasto}

Literature RePORTS: Domański 1984, Niemelä 2013.

Remarks. Domański (1984), following Parmasto (1968), reported the species from the BF but it was described based on material collected in the Belarusian part of the BF. We did not find it in the Polish part of the BF but Niemelä (2013) recently reported it based on a new record.

\section{Loweomyces fractipes (Berk. \& M. A. Curtis)} Jülich

Fig. 12A

Specimens eXamined: 417 (W. Szafer Landscape Reserve), on fallen branch, 15 Sept. 2011, Karasiński 6289 (D.K.).

LITERATURE REPORTS: None. The species is new for the $\mathrm{BF}$.

REMARKS. This may be a rare species in the study area or else undercollected due to its occurrence in wet places often inaccessible for collecting, flooded during the vegetative season. It was recorded only in the W. Szafer Landscape Reserve, outside the BNP borders. Basidiomata of L. fractipes are often stipitate or spathulate, and usually small. It grows on fine woody debris, especially fallen branches of deciduous trees lying on the ground in wet or periodically flooded places. Its basidiomata are often produced during periods when these habitats are temporarily dried. The species is slightly similar to Spongipellis delectans (Peck) Murrill, especially in the initial stages of basidiomata development. The latter inhabits coarse woody debris (often dead standing trees) and has significantly larger basidiomata with larger, sinuous pores, dissepiments becoming lacerate to dentate or even hydnoid with age, and longer basidia with larger basidiospores.

Fig. 12. A - Loweomyces fractipes (Berk. \& M. A. Curtis) Jülich, B - Mensularia radiata (Sowerby) Lázaro Ibiza, C - Oligoporus balsameus (Peck) Gilb. \& Ryvarden, D - Oligoporus ptychogaster (Ludwig) R. \& O. Falck, E - Oligoporus romellii (M. Pieri \& Rivoire) Niemelä, F - Oligoporus sericeomollis (Romell) Jülich, G - Onnia leporina (Fr.) H. Jahn, H - Onnia tomentosa (Fr.) P. Karst. Photo D. Karasiński (A-H). 

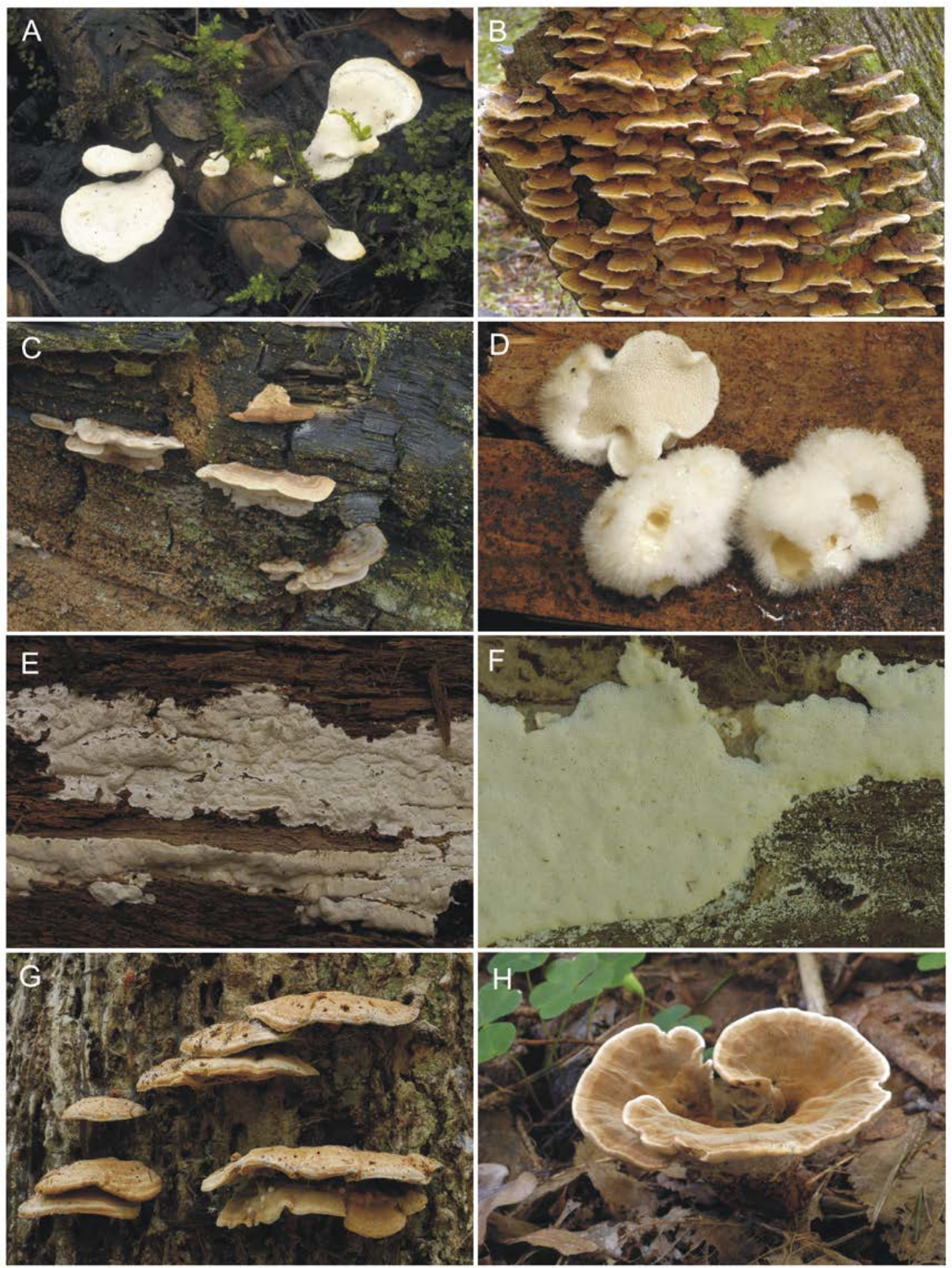
Mensularia radiata (Sowerby) Lázaro Ibiza

Fig. 12B

Specimens examined: 123C, on Carpinus dead standing trunk, 17 Oct. 2014, Karasiński 10993 (D.K.); 194D, on Alnus fallen branch, 30 July 2009, Karasiński $3669 D$ (D.K.); 214D (D.K. 11024); 225 (D.K. 3687E); 272 (Lipiny Reserve), on Corylus dead standing trunk, 21 May 2006, Karasiński 060521-2 (D.K.); 282 (D.K. 3520A); 340D, on Corylus avellana dead standing trunk, 7 July 2009, Karasiński 3276B (D.K.); 368A, on Corylus dead standing trunk, 13 Sept. 2009, Karasiński 4078A (D.K.); 369A, on deciduous fallen trunk, 13 Sept. 2009, Karasiński 3481 (D.K.); 374B, on Alnus dead standing trunk, 21 Sept. 2011, Karasiński 6438 (D.K.); 374C, on Corylus stump, 11 July 2009, Karasiński 3426A (D.K.); 375D (D.K. 10967); 399 (D.K. 5065B); 413A (H.M.W. M-1024); 413A (H.M.W. M-1213); 462A (H.M.W. M-1334); 516D (H.M.W. M-2134).

Literature REPORTs: Błoński et al. 1888 (as Polyporus radiatus), Błoński 1889a (as Ochroporus radiatus), Orłoś 1951 (as Polystictus radiatus), Orłoś 1961, Domański et al. 1967, Domański 1967, Anonymous 1968 (as Inonotus radiatus), Bujakiewicz et al. 1992 (as Inonotus radiatus), Bujakiewicz 1994 (as Inonotus radiatus), Jaroszewicz 1996 (as Inonotus radiatus), Grzywacz et al. 1996 (as Inonotus radiatus), Skirgiełło 1997 (as Inonotus radiatus), Orłoś 1960 (as Inonotus radiatus), Szczepkowski et al. 2008 (as Inonotus radiatus), Bujakiewicz \& Kujawa 2010 (as Inonotus radiatus), Gierczyk et al. 2013 (as Inonotus radiatus), Niemelä 2013 (as Inonotus radiatus).

REMARKS. In the study area it is a common species on Alnus and Corylus.

\section{Meripilus giganteus (Pers.) P. Karst.}

SPeCimens EXAMINED: 414B, at base of Quercus stump, 25 Aug 2001, Wołkowycki (H.M.W. M-1659).

LiterATURE REPORTS: Bujakiewicz et al. 1992, Skirgiełło 1997, Niemelä 2013.

REMARKs. This distinctive species with a large compound basidiome was confirmed in recent studies by only one collection from forest section $414 \mathrm{~B}$ in the western part of the BF in the vicinity of Hajnówka. According to local people it also occurs in the Palace Park in Białowieża. Niemelä (2013) reported it based on personal information from Anna Kujawa.
Obba rivulosa (Berk. \& M. A. Curtis) Miettinen \& Rajchenb.

SPeCimens EXAmined: BF, on fallen branch of Pinus, 25 Oct. 1955, S. Domański (KRAM F-SD 5445 as Physisporinus rivulosus).

LiteratURe REPORTS: Karasiński et al. 2009 (as Ceriporiopsis rivulosa), Niemelä 2013.

REMARKS. This is an extremely rare species in the study area and also in Europe. It is known from Finland, France, Italy and the former Yugoslavia (Ryvarden \& Melo 2014).

Oligoporus balsameus (Peck) Gilb. \& Ryvarden

Fig. 12C

SPECIMENS EXAMINED: 340F, on Quercus robur log, 6 July 2009, Karasiński 3295 (D.K.); 370C, on rotten Picea log, 15 Oct. 2009, Karasiński 4733 (D.K.).

LITERATURE REPORTS: Skirgiełło 1998 (as Tyromyces kymatodes), Niemelä 2013 (as Postia balsamea).

REMARKS. This species is rare in the study area. It is characterized by having pileate or effused-reflexed basidiomata with a faintly zonate pileus surface, thick-walled fusiform cystidia that are sparsely encrusted at the apex, and oblong ellipsoidal, slightly thick-walled basidiospores $3.5-5.5 \times 2.2-2.8 \mu \mathrm{m}$ (in specimens examined).

Oligoporus ptychogaster (Ludwig) R. \& O. Falck Fig. 12D

SPecimens examined: 272 (Lipiny Reserve), on Picea fallen trunk, 26 Sept 2006, Karasiński 060926-5868-teleomorph and anamorph (D.K.); 286, on Picea fallen trunk, 21 Sept. 2009, Karasiński 4371-teleomorph and anamorph (D.K.); 374C, on Pinus fallen branch, 15 Sept. 2009, Karasiński 4168 (D.K.); 697B (Starzyna Reserve), on Picea fallen log, 28 Sept. 2006, Karasiński 060928-6184 (D.K.).

LiteratURE REPORTS: Orłoś 1961 (as Leptoporus ptychogaster), Szczepkowski et al. 2008, Gierczyk et al. 2013 (as Postia ptychogaster), Niemelä 2013 (as Postia ptychogaster), Gierczyk et al. 2014 (as Postia ptychogaster). 


\section{Oligoporus romellii (M. Pieri \& Rivoire)}

Niemelä

Fig. 12E

Specimens examined: 340B, on Picea fallen branch, 20 Sept. 2009, Karasiński 4292 (D.K.); 340B, on Picea fallen branch, 20 Sept. 2009, Karasiński 4301 (D.K.); 370C, on rotten Picea fallen log, 15 Oct. 2009, Karasiński 4724 (D.K.); 370, Poprzeczny Tryb, east of 'Mogitki' graves, on Picea fallen tree in wet place, 11 Sept. 2009, Niemelä 8615 \& D. Schigel (KRA); 375B, on Picea rotten stump, 13 Oct. 2009, Karasiński 4663 (D.K.); 416 (W. Szafer Landscape Reserve), on Picea fallen trunk, 15 Sept. 2011, Karasiński 6297 (D.K.).

LITERATURE REPORTS: Niemelä 2013.

REMARKs. In the BF this species grows exclusively on Picea, unlike the similar O. sericeomollis (Romell) Jülich which seems locally to be associated with dead wood of Pinus. The morphological differences between these two species are small and mostly refer to differences in the shape and size of encrusted cystidia. As a rule, O. romellii has more slender and narrower cystidia (up to $6 \mu \mathrm{m}$ wide), often with almost thin to slightly thick walls, while $O$. sericeomollis produces cystidia up to $10 \mu \mathrm{m}$ wide with very thick walls up to $3.5 \mu \mathrm{m}$ in specimens examined. For more details see Pieri and Rivoire (2006) and Ryvarden and Melo (2014). Oligoporus balsaminus (Niemelä \& Y. C. Dai) Niemelä (= Postia balsamina Niemelä \& Y. C. Dai) seems to be the same as Oligoporus romellii.

Oligoporus sericeomollis (Romell) Jülich

Fig. $12 \mathrm{~F}$

Specimens eXamined: 284, on Pinus fallen log, 13 Sept. 2011, Karasiński 6285 (D.K.); 288, on Pinus fallen log, 23 Sept. 2011, Karasiński 6537 (D.K.); 289, on Pinus fallen log, 22 Sept. 2011, Karasiński 6451 (D.K.); 316, on Pinus fallen log, 23 Sept. 2011, Karasiński 6533 (D.K.); 316, on Pinus dead standing trunk, 24 Sept. 2011, Karasiński 6558 (D.K.); 374, on Pinus fallen log, 21 Sept. 2011, Karasiński 6407 (D.K.); 374D, on Pinus fallen log, 13 Oct. 2009, Karasiński 4646 (D.K.); 374D, on Pinus rotten fallen trunk, 24 Sept. 2010, Karasiński 6039 (D.K.); 375D, on Pinus big fallen log, 16 Oct. 2014, Karasiński 10971 (D.K.).

LiterATURE REPORTS: Domański 1965 (as Chaetoporellus litschaueri and Tyromyces sericeo-mollis), Domański 1967 (as Tyromyces sericeo-mollis),
Domański 1972b (as Strangulidium sericeo-molle), Niemelä 2013.

REMARKs: See remarks under Oligoporus romellii.

Onnia leporina (Fr.) H. Jahn

Fig. 12G

SPecimens eXAmined: 255D, on trunk of Picea living tree, 19 Sept. 2011, Karasiński 6349 (D.K.); 369, on living tree of Picea, 27 Aug. 1973, Wojewoda s.n. (KRAM F-34254); 417A, on Picea dead standing trunk, 28 Aug. 2012, Wolkowycki (H.M.W. M-0188); 417C (H.M.W. M-0189); 443, W. Szafer Landscape Reserve, on standing trunk of Picea, 29 Sept. 2006, Karasiński 1642 (KRAM F-47394); 500, in the vicinity of Podolany Reserve, on dead standing trunk of Picea, 27 Sept. 2006, Karasiński 1636 (KRAM F-47393); BF, on living tree of Picea, 25 Oct. 1959, Domański (KRAM F-SD 609 as Polystictis tomentosus var. triqueter).

LITERATURE REPORTS: Kotlaba \& Lazebniček 1967 (as Mucronoporus circinatus), Anonymous 1968 (as Mucronoporus tomentosus var. circinatus), Domański et al. 1973 (Mucronoporus tomentosus var. triqueter), Wojewoda 2003 (as Inonotus leporinus), Karasiński et al. 2009 (as Inonotus leporinus), Szczepkowski et al. 2011 (as Inonotus leporinus), Niemelä 2013 (as Pelloporus leporinus).

REMARKS. This is a rare species associated with Picea, on which it is a parasite and saprobe. Basidomata are mainly observed on bark of dead standing trees.

Onnia tomentosa (Fr.) P. Karst.

Fig. $12 \mathrm{H}$

SPecimens EXAmined: 285A, on the ground in mixed forest (Quercus, Picea, Carpinus), 29 July 2009, Karasiński 3659 (D.K.); 289C, on the ground in mixed forest under Picea, 12 Aug. 2009, Karasiński 3746e (D.K.); 315A, on roots of Picea stump, 18 Aug. 2009, Karasiński 3902 (D.K.); 369B, on base of dead standing trunk of Pinus, 13 Sept. 2011, Karasiński 6231 (D.K.); 699C (H.M.W. M-3613); BF, on the ground under Pinus, 18 Oct. 1960, Domański (KRAM F-SD 1373); BNP, Poprzeczny Tryb, east of 'Mogiłki' graves, on the ground in Carpinus-Picea-Quercus mixed forest, 11 Sept. 2009, Niemelä 8596 (KRA).

Literature REPORTS: Błoński et al. 1888 (as Polyporus tomentosus), Orłoś 1960 (as Polystictus tomentosus), Orłoś 1961 (as Polystictus tomentosus), Domański 1967, Anonymous 1968 (as Mucronoporus tomentosus), Bujakiewicz 2003, Bujakiewicz \& Kujawa 
2010, Szczepkowski et al. 2010 (as Inonotus tomentosus), Niemelä 2013 (as Pelloporus tomentosus).

Onnia triquetra (Pers.) Imazeki

Specimens eXamined: 284D, at base of Pinus dead standing trunk, 13 Sept. 2011, Karasiński 6263 (D.K.); 369B, on Pinus stump, 13 Sept. 2011, Karasiński 6231A (D.K.).

Literature REPORTS: Błoński 1889a (as Ochroporus triqueter), Siemaszko 1925 (as Polyporus circinatus), Domański et al. 1967 (as Mucronoporus tomentosus var. triqueter), Niemelä 2013 (as Pelloporus triquetrus).

REMARKS. This species is rare in the study area or undercollected.

Oxyporus corticola (Fr.) Ryvarden Fig. 13A

SPecimens EXAMINED: 98B (D.K. 10989B); 214D (D.K. 11025); 282D, on Populus fallen trunk, 25 July 2009, Karasiński 3522A (D.K.); 315B (D.K.3897); 315D (D.K. 3909); 346A (D.K. 3729); 369A, on Populus fallen trunk, 14 July 2009, Karasiński 3485 (D.K.); 369A, on necrotic swelling on trunk of dead standing Populus, 14 July 2009, Karasiński 3485A (D.K.); 372D, on Populus fallen trunk, 20 Aug. 2009, Karasiński 3978 (D.K.); 372D (D.K. 3972); 399D (D.K. 5053); 402A (D.K. 4952); 437D (H.M.W. M-0032).

LITERATURE REPORTS: Domański 1965 (as Chaetoporus corticola), Domański 1967, 1972b, Niemelä 2013, Gierczyk et al. 2014.

REMARKS. In the study area this is a rather common species showing a clear preference for Populus. The microscopically similar Oxyporus ravidus (Fr.) Bondartsev \& Singer produces pileate basidiomata and according to recent observations in the BF it grows on other hardwoods.

Oxyporus latemarginatus (Durieu \& Mont.) Donk

Fig. 13B

Specimens eXamined: 417 (W. Szafer Landscape Reserve), on bark of Populus fallen trunk, 20 Sept. 2013, Karasiński 9968 (D.K.).
Literature ReCORDs: None. The species is new for the BF.

REMARKs. This species is similar to Oxyporus obducens (Pers.) Donk but differs by having larger pores and basidiospores.

\section{Oxyporus obducens (Pers.) Donk}

SPeCimens EXAmined: 398B, on Ulmus fallen trunk, 14 Aug. 2009, Karasiński 3799 (D.K.).

Literature RePORTS: Błoński et al. 1888 (as Polyporus obducens), Domański 1965, 1972b, Niemelä 2013.

REMARKS. This species appears to be very rare in the study area. It has annual resupinate basidiomata with 4-6 pores per $\mathrm{mm}$, and small, ellipsoidal basidiospores 3.5-4.5 × 2.5-3 $\mu \mathrm{m}$ (in specimen examined). The morphologically similar $O$. corticola and O. latemarginatus have larger pores and basidiospores. Oxyporus populinus (Schumach.) Donk usually forms perennial, effused-reflexed basidiomata (rarely annual and resupinate), and has subglobose basidiospores.

Oxyporus populinus (Schumach.) Donk

Fig. 13C

SPeCimEns EXAMINED: 163C, on trunk of Acer platanoides living tree, 12 July 2009, Karasiński 3444 (D.K.); 221A (H.M.W. M-0156); 306C, at base of trunk of Acer platanoides living tree, 24 Mary 1993, Wołkowycki (H.M.W. M-1136); 316C, on trunk of Acer platanoides living tree, 27 Apr. 2010, Karasiński 5281A (D.K.); 372C, on trunk of Acer platanoides living tree, 5 July 2009, Karasiński 3253B (D.K.); 375B (D.K. 10969); 400B, on Acer platanoides large fallen branch, 5 July 2009, Karasiński 3262 (D.K.); 413C (H.M.W. M-1102); 439A (H.M.W. M-2000); 439C (H.M.W. M-0987); 463B (H.M.W. M-1496); 487B (H.M.W. M-1380).

Literature REPORTS: Siemaszko 1925 (as Polyporus connatus), Orłoś 1960, 1961, Domański et al. 1967, Domański 1967, Skirgiełło 1984, Bujakiewicz \& Kujawa 2010, Niemelä 2013.

REMARKS. All recent records are from Acer platanoides.

Fig. 13. A - Oxyporus corticola (Fr.) Ryvarden, B - Oxyporus latemarginatus (Durieu \& Mont.) Donk, C - Oxyporus populinus (Schumach.) Donk, D - Oxyporus ravidus (Fr.) Bondartsev \& Singer, E - Perenniporia medulla-panis (Jacq.) Donk, F - Perenniporia narymica (Pilát) Pouzar, G - Perenniporia subacida (Peck) Donk, H - Phaeolus schweinitzii (Fr.) Pat. Photo D. Karasiński (A-H). 

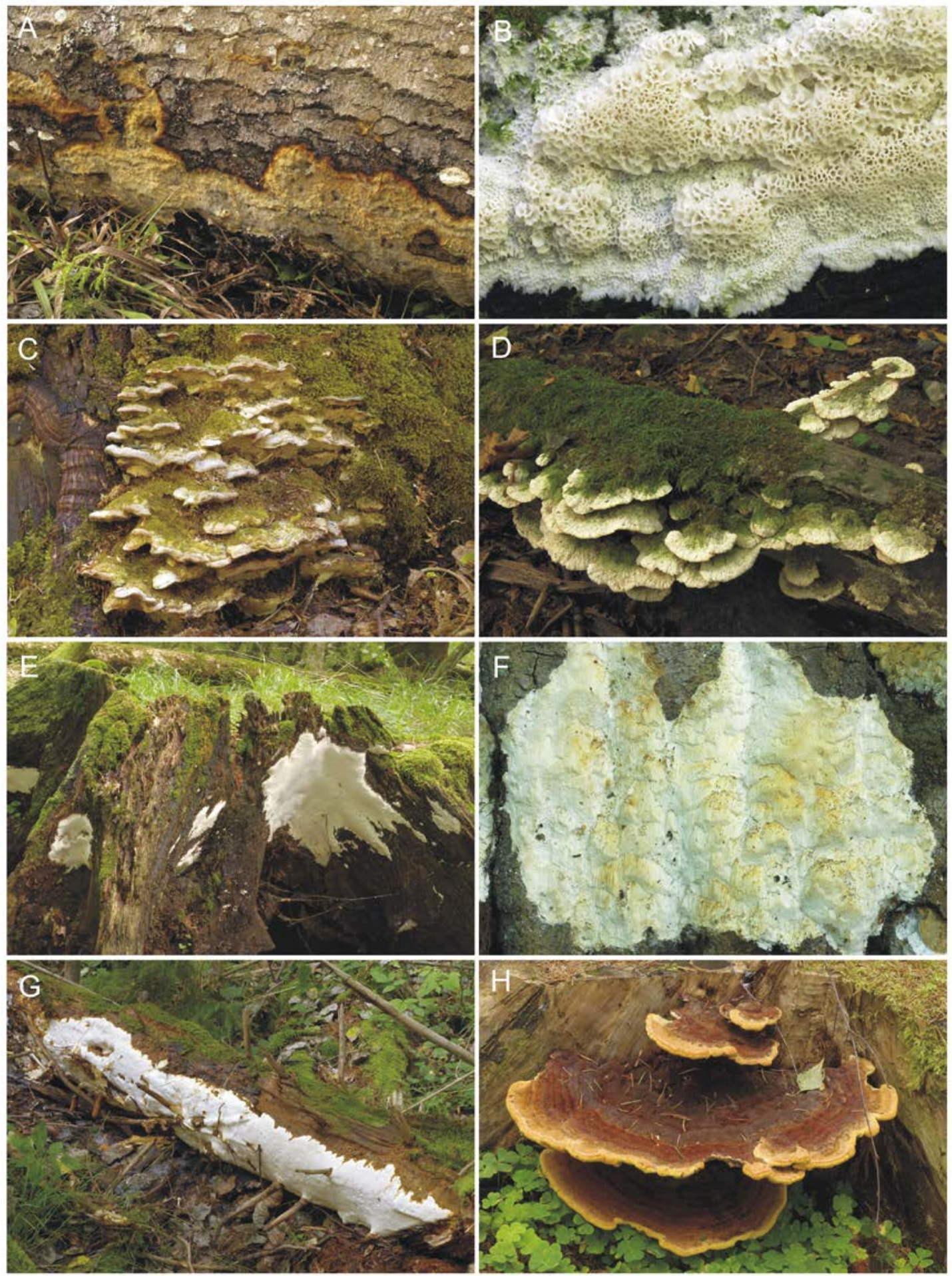
Oxyporus ravidus (Fr.) Bondartsev \& Singer

Fig. 13D

Specimens eXAmined: 194D, on Fraxinus fallen trunk, 30 July 2009, Karasiński 3663 (D.K.); 314C, on fallen trunk of deciduous tree (Carpinus?), 13 Sept. 2011, Karasiński 6288 (D.K.); 340, on Alnus fallen trunk, 20 Sept. 2009, Karasiński 3663 (D.K.).

Literature REPORTS: Domański 1965, 1967, Domański et al. 1967, Domański 1972b, 1973, Niemelä 2013.

REMARKs. This species is similar to Oxyporus corticola, but differs by having pileate or effusedreflexed basidiomata.

\section{Perenniporia medulla-panis (Jacq.) Donk}

Fig. 13E

SPECIMENS EXAMINED: 133B, on Quercus stump, 27 Apr. 2010, Wotkowycki (H.M.W. M-0081); 163C, on Quercus stump, 12 July 2009, Karasiński 3443 (D.K.); 194C, on Quercus stump, 9 July 2009, Karasiński 3363 (D.K.); 214D (D.K. 11021); 221A (H.M.W. M-0153); 226A, on Quercus stump, 30 July 2009, Karasiński 3679(D.K.); 239A (H.M.W. M-0111); 314C, on Quercus fallen log, 13 Sept. 2011, Karasiński 6237 (D.K.); 369B, on Quercus log, 13 Sept. 2011, Karasiński 6231 (D.K.); 374B, on Quercus fallen trunk, 15 Sept. 2009, Karasiński 4180 (D.K.).

Literature RePorts: Błoński et al. 1888 (as Polyporus medulla panis and Polyporus unitus), Błoński 1889a (as Polyporus medulla-panis), Domański 1965, 1967 (as Poria medullaris), Domański 1972b, Gierczyk et al. 2013, Niemelä 2013.

\section{Perenniporia narymica (Pilát) Pouzar Fig. 13F}

Specimens eXamined: 285A, on bark of Populus dead standing trunk, around and on Fomes fomentarius dead basidiome, 15 Aug. 2009, Karasiński 3822 (D.K.), the same locality and host tree, 19 Aug. 2009, Karasiński 3958 (D.K.); 372B, on decorticate Populus fallen trunk, 20 Aug. 2009, Karasiński 3990 (D.K.); 402, on Populus fallen trunk, 21 Sept. 2011, Karasiński 6402 (D.K.); 402, on Populus trunk, 22 Sept. 2011, Karasiński 6445 (D.K.); BNP, Sierchanowski Tryb, on Betula pubescens standing dead tree decayed by Fomes fomentarius, 14 Sept. 2009, Niemelä 8651 (KRA as Perenniporia subacida).

LiterATURE REPORTS: None. The species is new for the BF.
REMARKS. This species was previously reported from southeastern Poland by Domański (1973 as Poria elongata and Perenniporia elongata) based on a specimen collected in the Bieszczady National Park (KRAM F-SD 4820!). It appears to be a very rare Eurasian species associated with deciduous wood and old-growth forests. It is characterized by having annual, resupinate basidiomata, amyloid skelatal hyphae dissolving in $\mathrm{KOH}$, and ellipsoidal or ovoid basidiospores $4.0-5.5 \times 3.0-3.8 \mu \mathrm{m}$ (in specimens examined), often with a slightly concave adaxial side, negative in Melzer's reagent. The genus Yuchengia B. K Cui \& Steffen was recently proposed to accommodate this species (Zhao et al. 2013).

\section{Perenniporia subacida (Peck) Donk Fig. 13G}

SPECIMENS EXAMINED: 225 (D.K. 6396); 226A (D.K. 3692); 254D (D.K. 3806); 256A, on Picea fallen trunk, 29 July 2009, Karasińśki 3645 (D.K.); 285A (D.K. 3954); 285A (D.K. 5300); 314C (D.K. 5182); 317C, on Picea rotten trunk, 14 Aug. 2009, Karasiński 3787 (D.K.); 340C (D.K. 4320); 340G (D.K. 4410); 346D, on Picea fallen trunk, 27 July 2009, Karasiński 3581 A (D.K.); 346D (D.K. 4687); 374D, on Pinus log, 26 July 2009, Karasiński 3549 (D.K.); 399B, on Picea fallen log, 5 July 2009, Karasiński 3243 (D.K.).

Literature REPORTS: Domański 1964b, 1965 (as Poria subacida), Domański 1967, Domański 1972b, Szczepkowski et al. 2010, Niemelä 2013.

REMARKs. This species is characterized by having very large, resupinate and perennial basidiomata, with a distinctly stratified context, strongly dextrinoid skeletal hyphae up to $5 \mu \mathrm{m}$ in diam., slender binding hyphae, and ovoid to ellipsoidal, often weakly dextrinoid basidiospores $4.8-6.5 \times$ $3.8-4.6 \mu \mathrm{m}$. In the BF it grows almost exclusively on Picea, once observed on Pinus. The species is very rare in Poland, known only from two localities - the BF and the Augustów Forest (Holec \& Pouzar 1998; Wojewoda 2003).

Phaeolus schweinitzii (Fr.) Pat.

Fig. $13 \mathrm{H}$

SPeCimens EXAmined: 398, on trunk of Picea, 21 Sept. 2007, Karasiński 070921-7775 (D.K.); 412A, at base of Pinus dead standing trunk, 9 Sept. 2001, Wolkowycki (H.M.W. M-1749); 416B (H.M.W. 
M-1386); 500A (Podolany Reserve), on the ground under Picea, 27 Sept. 2006, Karasiński 060927-6068 (D.K.); BF, on Picea trunk, 29 Apr. 1960, Domański (KRAM F-SD 626).

Literature REPORTs: Błoński 1889a (as Polyporus spongia), Orłoś 1960, 1961, Domański et al. 1967, Domański 1967, Bujakiewicz et al. 1992, Jaroszewicz 1996, Skirgiełło 1997, Gierczyk et al. 2013, Niemelä 2013.

\section{Phellinidium ferrugineofuscum (P. Karst.)}

Fiasson \& Niemelä Fig. 14A

SPeCimenS EXAMINED: 135D, on bark of Picea fallen trunk, 8 July 2009, Karasiński 3336 (D.K.); 194D (D.K. 3665C1); 225B (D.K. 3685); 255D (D.K. 5218); 261A (D.K. 3376, DK 3388 DK 3393); 285A (D.K. 5330A); 285C (D.K. 5287B); 314A (D.K. 3304); 315B (D.K. 3913); 316C (D.K. 5260E); 318D (D.K. 3879); 319C (D.K. 3607); 340B (D.K. 4223A); 340G (D.K. 4225); 340G (4305A); 346D (D.K. 4679A); 369A (D.K. 3490); 369C (4374); 370C (D.K. 3703D); 373D (D.K. 4679A, DK 4994); 374D (D.K. 3534A); 375B (D.K. 3579); 399C, on bark of Picea fallen log, 1 Nov. 2009, Karasiński 5022 (D.K.).

LiTERATURE REPORTS: Domański 1965, 1967, Kotlaba \& Lazebniček 1967, Anonymous 1968, Domański 1972b, Szczepkowski et al. 2010, Niemelä 2013. All literature records are as Phellinus ferrugineofuscus.

REMARKs. The species is associated with dead wood of Picea in an early stage of decomposition. It is characterized by having annual to biennial, resupinate basidiomata with small pores 7-9 per $\mathrm{mm}$, long, tramal setae, lacking hymenial setae and having allantoid basidiospores. Phellinidium ferrugineofuscum was collected many times during recent studies and seems to be quite common in the study area. However, in Poland it is so far not known outside the BF (Wojewoda 2003).

\section{Phellinopsis conchata (Pers.) Y. C. Dai} Fig. 14B

Specimens eXAmined: 402B, on Salix caprea fallen trunk, 26 July 2009, Karasiński 3532 (D.K.); 414B, on trunk of Salix caprea, 10 Feb. 1998, Wolkowycki (H.M.W. M-0881); 442B, on Salix caprea fallen trunk, 10 Sept. 2004,Wotkowycki (H.M.W. M-3120).

LITERATURE REPORTS: Orłoś 1960 (as Phellinus con- chatus), Gierczyk et al. 2013 (as Phellinus conchatus), Niemelä 2013 (as Phellinus conchatus).

REMARKS. This species appears to be rare in the study area or undercollected.

Phellinus alni (Bondartsev) Parmasto Fig. 14C

Specimens EXAmined: 123D (D.K. 10994); 214B (Szczekotowo Reserve), on trunk of Carpinus living tree, 17 Oct. 2014, Karasiński 11005 (D.K.); 285A, on Carpinus living tree, 28 Apr. 2010, Karasiński 5326B (D.K.); 314A, on trunk of Carpinus living tree, $25 \mathrm{Apr}$. 2010, Karasiński 5197A (D.K.); 316C, on Carpinus living tree, 27 Apr. 2010, Karasiński 5279A (D.K.); 438A (H.M.W. M-0068); 439A (Głęboki Kąt Reserve), on Alnus fallen trunk, 14 Sept. 2005, Karasiński 0509147721 (D.K.).

LITERATURE REPORTS: Niemelä 2013.

Phellinus igniarius (L.) Quél.

Fig. 14D

SPECIMENS EXAMINED: 484A (Lozice), on trunk of Salix fragilis living tree, 23 March 1993, Wotkowycki (H.M.W. M-1027); BF, near bank of the Hwoźna River, on Salix fallen branch, 23 Sept. 1965, Domański (KRAM F-SD 4876).

Literature RePORTS: Błoński et al. 1888 (as Polyporus igniarius), Błoński 1889a (as Ochroporus igniarius), Karpiński 1949 (as Polyporus igniarius), Orłoś 1951 (as Fomes igniarius), Orłoś 1955b, Orłoś 1960, Orłoś 1961, Orłoś \& Twarowska 1967, Domański et al. 1967, Domański 1967, Anonymous 1968, Nespiak 1968, 1970, Bujakiewicz et al. 1992, Jaroszewicz 1996, Grzywacz et al. 1996, Skirgiełło 1997, 1998, Bujakiewicz \& Kujawa 2010, Gierczyk et al. 2013, Niemelä 2013.

REMARKS. Some specimens given in literature reports may in fact represent other species from the Phellinus igniarius complex, e.g., P. alni, $P$. nigricans (Fr.) P. Karst. or P. populicola Niemelä. Among several specimens collected in the BF and preserved in Domański's herbarium only one (cited above) belongs to Phellinus igniarius s.str.

\section{Phellinus laevigatus (Fr.) Bourdot \& Galzin}

Fig. 14E

SPECIMENS EXAMINED: 194D, on bark of Betula pubescens fallen trunk, 30 July 2009, Karasiński 3661 (D.K.); 261A, on Betula pendula fallen trunk, 10 July 2009, Karasiński 3373 (D.K.); 318B, on Betula sp. 
fallen trunk, 12 Aug. 2009, Karasiński 3744c (D.K.); 318D (H.M.W. M-2804); 369C, on Betula pendula fallen trunk, 22 Sept. 2009, Karasiński 4381 (D.K.); 402A, on Betula pendula fallen trunk, 31 Oct. 2009, Karasiński 4972 (D.K.); 437B, on Betula pendula fallen trunk, 20 May 2006, Karasiński 060520-5131 (D.K.); 437B (H.M.W. M-0024).

Literature RePORTs: Pilát 1950, Domański 1965, 1967, 1972b, Niemelä 1972, 2013, Gierczyk et al. 2014.

Phellinus nigricans (Fr.) P. Karst. Fig. 14F

SPeCimens exAmined: 314B, on trunk of Betula pendula living tree, 18 Aug. 2009, Karasiński 3888 (D.K.); 440, on Betula pendula fallen trunk, 23 May 2006, Karasiński 060523-5488 (D.K.).

Literature REPORTS: None. This species is new for the BF.

REMARKS. This species appears to be rare in the study area and in its strict sense it was previously not reported from the BF. Among specimens of Phellinus igniarius s.1. deposited in KRAM F-SD there is no specimen that fit the genuine Phellinus nigricans.

\section{Phellinus populicola Niemelä}

Fig. 14G

SPeCimens EXAmined: 98B (D.K. 10980); 224B (D.K. 3946A, DK 3950A); 256D (D.K. 5240A); 282D, on trunk of old Populus living tree, 25 July 2009, Karasiński 3524b (D.K.); 314B (D.K. 3885B, 3907B); 315C (D.K. 3928B); 316A (D.K. 5280A); 318B, on trunk of Populus living tree, 12 Aug. 2009, Karasiński 3744e (D.K.); 340C (D.K. 4319C); 369A, on trunk of Populus living tree, 14 July 2009, Karasiński 3480 b (D.K.); 370A (D.K. 4400A); 370D (D.K. 4737); 371D (D.K. 3252); 372D (D.K. 3966A); 399C (D.K. 5046); 402A, on trunk of Populus living tree, 13 July 2009, Karasiński 3479a (D.K.); 443A (W. Szafer Landscape Reserve), on Populus dead standing trunk, 23 June 2008, Karasiński 1689 (D.K.).

LITERATURE REPORTS: Niemelä 1975, Szczepkowski et al. 2008, 2011, Niemelä 2013, Gierczyk et al. 2013, 2014.

REMARKS. This is a fairly common species in old aspen stands. During recent studies it was recorded many times. Outside the BF it is very rare in Poland, known from only a few sites (Wojewoda 2003).

Phellinus tremulae (Bondartsev) Bondartsev \& Borissov

Fig. $14 \mathrm{H}$

SPECIMENS EXAMINED: 98B (D.K. 10984, D.K. 10989); 197B (D.K. 3370D); 197D (D.K. 3370E); 225A (D.K. 3687A); 226A, on trunk of Populus living tree, 9 July 2009, Karasiński 3355 (D.K.); 255C (D.K. 5242A); 256D (D.K. 5240B); 261A, on trunk of old Populus living tree, 10 July 2009, Karasiński 3381 (D.K.); 282D (D.K. 3527B); 315B (D.K. 3907A); 315D (D.K. 3921A); 315D (D.K. 5251); 316A (D.K. 5280), 340G (D.K. 4224A); 342C (D.K. 4791); 369A (D.K. 3480C); 370B (D.K. 4799A); 372D (D.K. 3966C); 398G (D.K. 3498); 399C (D.K. 5056A); 402A (D.K. 3479A).

LITERATURE REPORTS: Pilát 1950 (as Phellinus igniarius var. tremulae), Orłoś 1960 (as Phellinus igniarius var. tremulae), Jaroszewicz 1996, Grzywacz et al. 1996, Szczepkowski et al. 2008, Niemelä 2013, Gierczyk et al. 2013, 2014.

\section{Phellinus tuberculosus (Baumq.) Niemelä}

SPECIMENS EXAMINED: Białowieża village (Zastawa), on trunk of Prunus domestica in orchard, 20 July 1990, Wotkowycki (H.M.W. M-1026); Masiewo village, on Prunus domestica fallen branch, 16 Nov. 2006, Wotkowycki (H.M.W. M-0045).

LiTERATURE REPORTS: Niemelä 2013.

REMARKS. This species was observed on cultivated Prunus domestica L. in anthropogenic habitats outside forest.

Phellopilus nigrolimitatus (Romell) Niemelä, T. Wagner \& M. Fisch.

Fig. 15A

SPecimens EXAmined: 254D, on rotten wood of Picea fallen log, 15 Aug. 2009, Karasiński 3804A (D.K.); 255D (D.K. 5217), 256A (D.K. 3638); 256D, 257C (D.K. 4339A); 258C (D.K. 4361); 283C, 284B (D.K. 3814E); 284D (D.K. 5331); 285A (D.K. 5314A); 285C, 288D, on Picea fallen trunk, 12 Aug. 2009, Karasiński

Fig. 14. A - Phellinidium ferrugineofuscum (P. Karst.) Fiasson \& Niemelä, B - Phellinopsis conchata (Pers.) Y. C. Dai, C Phellinus alni (Bondartsev) Parmasto, D - Phellinus igniarius (L.) Quél., E - Phellinus laevigatus (Fr.) Bourdot \& Galzin, F - Phellinus nigricans (Fr.) P. Karst., G - Phellinus populicola Niemelä, H - Phellinus tremulae (Bondartsev) Bondartsev \& Borissov. Photo D. Karasiński (A-H). 


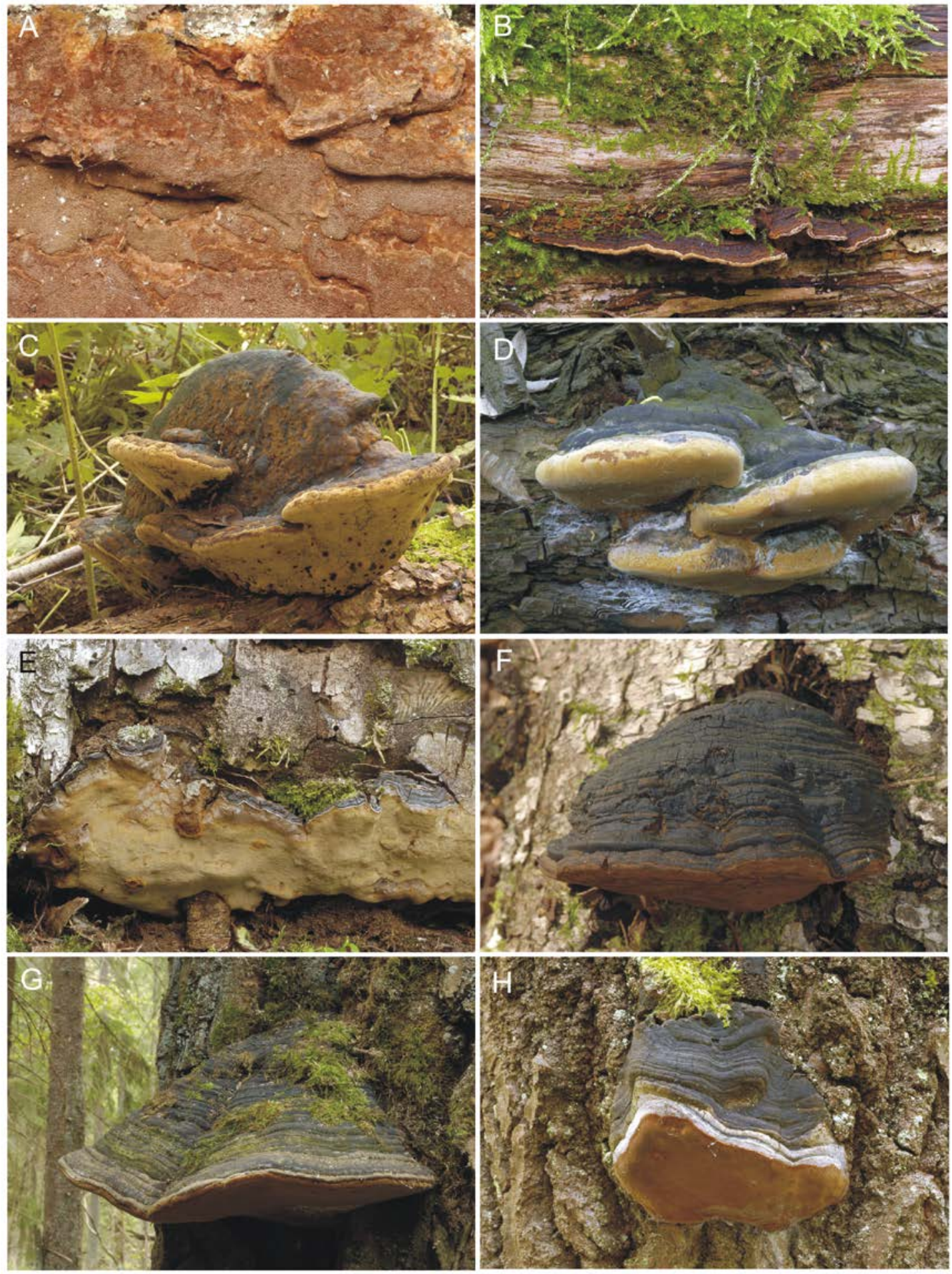


3743D (D.K.); 314C (D.K. 5175, DK 3306); 314D (D.K. 5196B); 315A (D.K. 3893B); 316C (D.K. 5275); 319C (D.K. 3748), 340B (3278A); 340F (D.K. 4197A1); 341B (D.K. 4407); 341C (D.K. 4767B); 341D (5251B), 344A (D.K.3836); 344B (D.K. 3854); 346D (D.K. 3582); 346D (D.K. 4688); 369A (D.K. 3480A); 370C (D.K. 3698D); 373B (D.K. 3460D); 373D (D.K. 3449A); 374C (D.K. 3408D); 374D (D.K. 3415B); 374D (D.K. 3534C); 375A (D.K. 4285A); 398G,on Picea fallen trunk, 24 July 2009, Karasiński 3498A (D.K.); 399A (D.K. 5028); 399B (D.K. 5034); 399C (D.K. 5072); 400A (D.K. 5339A); 402B (H.M.W. M-3654).

Literature RePORTs: Pilát 1950, Orłoś 1960, Domański 1962, 1965, Domański et al. 1967, Domański 1967, Kotlaba \& Lazebniček 1967, Anonymous 1968, Domański 1972b, Domański et al. 1973, Bujakiewicz et al. 1992, Skirgiełło 1997, Niemelä et al. 2001, Bujakiewicz 2003, Niemelä 2005, Szczepkowski et al. 2008, Bujakiewicz \& Kujawa 2010, Szczepkowski et al. 2011, Gierczyk et al. 2013, Niemelä 2013, Gierczyk et al. 2014. All literature records (except Niemelä et al. 2001) are as Phellinus nigrolimitatus.

Physisporinus sanguinolentus (Alb. \& Schwein.) Pilát Fig. 15B

SPecimens EXAmined: 340A, on rotten wood of base of dead standing Quercus robur trunk, 22 Sept. 2010, Karasiński 6003 (D.K.); 340F, on Picea rotten stump, 16 Sept. 2009, Karasiński 4234 (D.K.); 340G, on fallen branch of deciduous tree (Fraxinus?), 16 Sept. 2009, Karasiński 4204a (D.K.); 375A, on Picea stump, 19 Sept. 2009, Karasiński 4283 (D.K.); 387 (W. Szafer Landscape Reserve), on rotten trunk, 20 Sept. 2013, Karasiński 9970 (D.K.).

LiTERATURE REPORTS: Domański 1965, 1967 (as Podoporia sanguinolenta), Anonymous 1968 (as Podoporia sanguinolenta), Domański 1972b (as Rigidoporus sanguinolentus), Szczepkowski et al. 2008, Niemelä 2013 (as Rigidoporus sanguinolentus).

Physisporinus vitreus (Pers.) P. Karst. Fig. 15C

Specimens examined: 245D, on Picea fallen trunk, 12 Sept. 2011, Karasiński 6211 (D.K.); 315B, on rotten Picea fallen trunk, 18 Aug 2009, Karasiński
3914 (D.K.); 340B, on rotten trunk of deciduous tree, 20 Sept. 2009, Karasińśki 4308 (D.K).

LiterATURE REPORTS: Domański 1967 (as Podoporia vitrea), Gierczyk et al. 2013, Niemelä 2013 (as Rigidoporus sanguinolentus), Gierczyk et al. 2014.

REMARKs. Microscopically similar Rigidoporus undatus (Pers.) Donk has tramal cystidia with small caps of apical encrustation, smaller pores, larger basidiospores and more robust basidiomata.

\section{Piptoporus betulinus (Bull.) P. Karst.}

Specimens eXamined: 98B, on Betula dead standing trunk, 17 Oct. 2014, Karasiński 10979 (D.K.); 375D, on Betula fallen trunk, 16 Oct. 2014, Karasiński 10974 (D.K.); 413A (Sacharewo), on trunk of Betula pendula, 25 Sept. 1999, Wołkowycki (H.M.W. M-1903); 413A (Sacharewo), on trunk of Betula pubescens, 10 Oct. 1998, Wolkowycki (H.M.W. M-1236); 463D (Bereźniaki), on trunk of Betula pendula, 16 March 1993, Wotkowycki (H.M.W. M-1098).

Literature RePORTs: Błoński et al. 1888 (as Polyporus betulinus), Błoński 1889a (as Polyporus betulinus), Siemaszko 1925 (as Polyporus betulinus), Karpiński 1949 (as Polyporus betulinus), Nespiak 1956 (as Polyporus betulinus), Orłoś 1960, 1961, Domański 1967, Anonymous 1968, Nespiak 1968, Skirgiełło 1976, Bujakiewicz et al. 1992, Jaroszewicz 1996, Grzywacz et al. 1996, Skirgiełło 1997, Szczepkowski et al. 2008, Bujakiewicz \& Kujawa 2010, Gierczyk et al. 2013, Niemelä 2013, Gierczyk et al. 2014.

Polyporus arcularius (Batsch) Fr. Fig. 15D

SPECIMENS EXAMINED: 226A, on Corylus avellana fallen branch, 30 July 2009, Karasiński 3672 (D.K.); 316C, on Carpinus fallen branch, 27 Apr. 2010, Karasiński 5282 (D.K.); BF, on hardwood, 1 May. 1959, Domański (KRAM F-SD 611); BF, on Carpinus fallen trunk, 28 Oct. 1956, Domański (KRAM F-SD 610).

LiterATURE REPORTS: Bujakiewicz et al. 1992, Bujakiewicz 1994, Skirgiełło 1997, Orłoś 1960 (as Polyporellus arcularius), Bujakiewicz \& Kujawa 2010, Niemelä 2013.

Fig. 15. A - Phellopilus nigrolimitatus (Romell) Niemalä, T. Wagner \& M. Fisch., B - Physisporinus sanguinolentus (Alb. \& Schwein.) Pilát, C - Physisporinus vitreus (Pers.) P. Karst., D - Polyporus arcularius (Batsch) Fr., E - Polyporus badius (Pers.) Schwein., F - Polyporus ciliatus Fr., G - Polyporus melanopus (Pers.) Fr., H - Polyporus tuberaster (Jacq.) Fr. Photo D. Karasiński $(\mathrm{A}-\mathrm{H})$. 

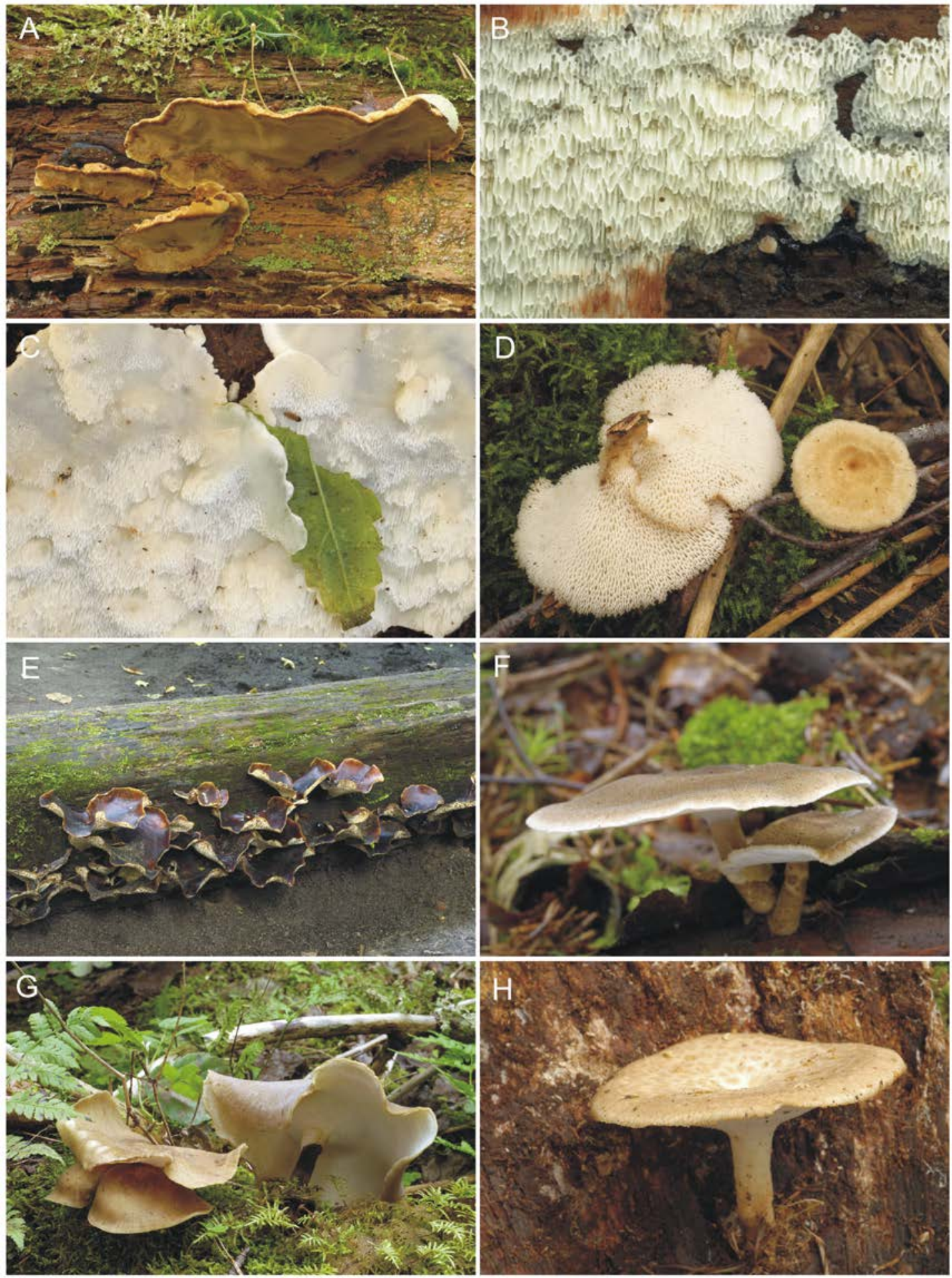


\section{Polyporus badius (Pers.) Schwein. Fig. 15E}

Specimens examined: 214A (H.M.W. M-1473); 369A, on fallen branch of deciduous tree, 14 July 2009, Karasiński 3481C (D.K.); 398B, on Ulmus sp. fallen trunk, 14 Aug. 2009, Karasiński 3799 (D.K.); 413B (H.M.W. M-1645); 572 (Michnówka Reserve), on deciduous fallen trunk, 24 May 2006, Karasiński 060524-5518 (D.K.); 414D (H.M.W. M-1458); 487B (H.M.W. M-1624); 633C (H.M.W. M-0927).

Literature RePORTS: Błoński et al. 1888 (as Polyporus picipes), Błoński 1889a (as Polyporus picipes), Siemaszko 1923 (as Polyporus picipes), Orłoś 1951 (as Polyporus picipes), Nespiak 1959 (as Polyporus picipes), Orłoś 1960, 1961 (as Polyporellus picipes), Domański et al. 1967 (as Polyporus picipes), Domański 1967 (as Polyporus picipes), Anonymous 1968 (as Polyporus picipes), Nespiak 1968 (as Polyporus picipes), Domański et al. 1973, Bujakiewicz et al. 1992, Bujakiewicz 1994, Jaroszewicz 1996 (as Polyporus picipes), Skirgiełło 1997, 1998, Szczepkowski et al. 2008, Bujakiewicz \& Kujawa 2010, Gierczyk et al. 2013, Niemelä 2013, Gierczyk et al. 2014.

\section{Polyporus brumalis (Pers.) Fr.}

Specimens examined: 214D (D.K. 11018); 272D (H.M.W. M-0555); 306A (H.M.W. M-0915); 329D (H.M.W. M-0738); 334D (H.M.W. M-0921); 355A (H.M.W. M-0906); 356A, on Corylus fallen trunk, 12 Dec. 1992, Wolkowycki (H.M.W. M-0898); 362A (H.M.W. M-0903); 367C (H.M.W. M-0883); 383A (H.M.W. M-0889); 384B (H.M.W. M-0894); 385A (H.M.W. M-0922); 402A (H.M.W. M-0577); 403A (H.M.W. M-0919); 404C (H.M.W. M-0900); 413B (H.M.W. M-1861); 414A (H.M.W. M-1862); 416C (H.M.W. M-0878); 437D (H.M.W. M-1871); 438A (H.M.W. M-1298); 439D (H.M.W. M-3047); 440A (H.M.W. M-0908); 461B (H.M.W. M-0977); 462B (H.M.W. M-0910); 463D (H.M.W. M-0231); 464A (H.M.W. M-0892); 466C (H.M.W. M-0252); 476A (H.M.W. M-0193); 486B (H.M.W. M-0913); 488C (H.M.W. M-0975); 501C (H.M.W. M-0905); 537B (H.M.W. M-0904); 575A (H.M.W. M-2817); 596C (H.M.W. M-0885); 628B (H.M.W. M-0891); 632A (H.M.W. M-0917); 661B (H.M.W. M-0736); 694C (H.M.W. M-0887); 725A (H.M.W. M-0739); BF, on rotten hardwood, 1 May 1959, Domański (KRAM F-SD 2691 as Polyporus subarcularius); BF, on fallen branch of deciduous tree, 29 Apr. 1960, Domański (KRAM F-SD 1262 as Polyporus subarcularius).

LITERATURE REPORTS: Błoński et al. 1888, Błoński 1889a (as Polyporus fuscidulus), Nespiak 1959, Orłoś 1960 (as Polyporellus brumalis), Bujakiewicz et al. 1992, Jaroszewicz 1996, Skirgiełło 1997, Niemelä 2013.

\section{Polyporus ciliatus Fr.}

Fig. 15F

SPeCimens EXAmined: 340D, on Tilia fallen branch, 6 July 2009, Karasiński 3275 (D.K.); 384D (H.M.W. M-0974); 412D, on well-rotted wood remnants, 7 July 2003, Wotkowycki (H.M.W. M-3015); 437B (H.M.W. M-0040); 442B (H.M.W. M-2013); 513B (H.M.W. $\mathrm{M}-1427)$.

Literature Reports: Domański et al. 1967, Domański 1967, Domański et al. 1973, Bujakiewicz et al. 1992, Bujakiewicz 1994, Jaroszewicz 1996, Skirgiełło 1997, 1998, Bujakiewicz \& Kujawa 2010.

Polyporus melanopus (Pers.) Fr.

Fig. $15 \mathrm{G}$

SPECIMENS EXAMINED: 261A, on well rotted trunk of deciduous tree covered by mosses, 10 July 2009, Karasiński 3382 (D.K.); 282D, on fallen twig of deciduous tree, 25 July 2009, Karasiński 3524a (D.K.); 313B, on fallen deciduous twig, 25 July 2009, Karasiński $3524 b$ (D.K.).

LiTERATURE REPORTS: Domański et al. 1967, 1973, Bujakiewicz et al. 1992, Bujakiewicz 1994, Skirgiełło 1997, Niemelä 2013.

\section{Polyporus squamosus (Huds.) Fr.}

SPECIMENS EXAMINED: 412D, on Corylus avellana fallen trunk, 7 July 2003, Wolkowycki (H.M.W. M-3013); 414D, on fallen trunk, 3 June 2001, Wotkowycki (H.M.W. M-2055); 485B, on fallen trunk, 11 June 2003, Wotkowycki (H.M.W. M-3012); BF, on Acer trunk, 9 Aug. 1962, Domański (KRAM F-SD 4198).

LiterATURE REPORTS: Błoński et al. 1888, Błoński 1889a, Karpiński 1949, Orłoś 1960 (as Polyporellus squamosus), Domański 1967, Bujakiewicz et al. 1992, Skirgiełło 1997, 1998, Niemelä 2013, Gierczyk et al. 2014.

Polyporus tuberaster (Jacq.) Fr.

Fig. $15 \mathrm{H}$

SPECIMENS EXAMINED: 226, on fallen branch of deciduous tree, 2001, Bujakiewicz (KRAM); 313A, on very rotten wood of deciduous tree, 25 July 2009, Karasiński 3505 (D.K.); 369A, on Acer platanoides fallen trunk, 14 July 2009, Karasiński $3481 b$ (D.K.); 464C, on Salix caprea fallen trunk, 22 Aug. 1999, Wotkowycki (H.M.W. M-1396); BF, on Carpinus fallen 
branch, 10 June 1966, Domański (KRAM F-SD 4993 as Polyporus coronatus).

Literature Reports: Domański 1967 (as Polyporus coronatus), Domański et al. 1973 (as Polyporus floccipes), Bujakiewicz 2003, Szczepkowski et al. 2008, Bujakiewicz \& Kujawa 2010, Niemelä 2013.

\section{Polyporus umbellatus (Pers.) Fr.}

Specimens eXAmined: 319C, on the ground under Carpinus, Quercus and Tilia, 13 Aug. 2009, Karasiński 3761 (D.K.).

Literature RePORTS: Błoński et al. 1888 (as Polyporus umbellatus), Błoński 1889a (as Polyporus ramosissimus), Orłoś 1955b, Domański et al. 1967 (as Grifola umbellata), Domański et al. 1973, Skirgiełło 1976 (as Grifola umbellata), Bujakiewicz et al. 1992 (as Grifola umbellata), Skirgiełł 1997 (as Grifola umbellata), Niemelä 2013.

\section{Polyporus varius (Pers.) Fr.}

Specimens examined: 356C, on Salix caprea, 23 Apr. 1993, Wolkowycki (H.M.W. M-0926); 439C (in the vicinity of Głęboki Kąt Reserve), on wood, 26 Nov. 2003, Wotkowycki (H.M.W. M-3050).

Literature RePORTS: Błoński et al. 1888 (as Polyporus elegans and P. varius), Błoński 1889a (as Polyporus elegans and P. varius), Domański et al. 1967 (as Polyporus varius var. nummularius), Domański 1967, Anonymous 1968, Nespiak 1968, Jaroszewicz 1996, Skirgiełło 1998, Bujakiewicz \& Kujawa 2010, Niemelä 2013 (as Polyporus leptocephalus).

Porodaedalea chrysoloma ((Fr.) Fiasson \& Niemelä

Fig. 16A

SPECIMENS EXAmined: 158B, on Picea fallen trunk, 20 Sept. 2007, Karasiński 070920-1 (D.K.); 226A (D.K.3678); 239A (H.M.W. M-0110); 257D, ca 30 basidiomata on trunk of Picea living tree, 21 Sept. 2009, Karasiński 4345 (D.K.); 313B (D.K. 3516); 316 (D.K. 5256); 334D (H.M.W. M-1057); 346D, on Picea branch, 13 Oct. 2009, Karasiński 4694 (D.K.); 373D (D.K. 4997); 399A, in necrotic depression on trunk of Picea living tree, 4 July 2009, Karasiński 3242 (D.K.); 438A (H.M.W. M-1056); 439A (H.M.W. M-3591); 463B (H.M.W. M-1493); 488C (H.M.W. M-1029); 572 (Michnówka Reserve), on Picea stump, 24 May 2006, Karasiński 060524-5548 (D.K.).

Literature REPORTS: Siemaszko 1923 (by mis- take as Polyporus annosus, misidentification revised in Siemaszko 1925 as Trametes abietis), Pilát 1950 (as Phellinus abietis), Domański et al. 1967 (as Phellinus pini var. abietis), Domański 1967 (as Phellinus pini var. abietis), Domański 1972b (as Phellinus chrysoloma), Szczepkowski et al. 2008 (as Phellinus chrysoloma), Niemelä 2013 (as Phellinus chrysoloma).

\section{Porodaedalea pini (Brot.) Murrill Fig. 16B}

Specimens eXamined: 130, on trunk of Pinus living tree, 14 Sept. 2009, Karasiński 4109a (D.K.); 224D, on Pinus living tree of, 19 Aug. 2009, Karasiński 3945a (D.K.); 255 (D.K. 5233) 260C, on trunk of Pinus living tree, 10 July 2009, Karasiński 3371 (D.K.); 272D (H.M.W. M-1059); 285 (D.K. 5299A); 318B (D.K. 3744F); 374C (D.K. 4165); 375A (D.K. 4282A); 375A (D.K. 4286A).

LiterATURE REPORTS: Błoński et al. 1888 (as Trametes pini), Karpiński 1949 (as Trametes pini), Pilát 1950, Orłoś 1955b, 1960, 1961, Orłoś \& Twarowska 1967, Domański et al. 1967, Domański 1967, Anonymous 1968, Jaroszewicz 1996, Grzywacz et al. 1996, Szczepkowski et al. 2008, Bujakiewicz \& Kujawa 2010, Szczepkowski et al. 2011, Niemelä 2013. All literature records since 1950 published as Phellinus pini.

Porotheleum fimbriatum (Pers.) Fr. Fig. 16C

SPECIMENS EXAMINED: 98B, on Betula fallen trunk, 17 Oct. 2014, Karasiński 10981 (D.K.); 402B, on Populus fallen log, 26 July 2009, Karasiński 3530 (D.K.); 451B ('Żebra Żubra' tract), on bark of Populus fallen trunk, 20 Sept. 2007, Karasiński 070920-1 (D.K.).

Literature REPORTs: Pilát 1950, Domański 1967 (as Stromatoscypha fimbriata).

Porpomyces mucidus (Pers.) Jülich Fig. 16D

SPecimens eXAmined: 226A, on very rotten Picea stump, 11 Oct. 2009, Karasiński 4578 (D.K.); 255D, on strongly decayed Picea log, 14 Aug. 2009, Karasiński 3768 (D.K.); 314C, on well-rotted deciduous log (Fraxinus?), 25 Apr. 2010, Karasiński 5173 (D.K.); 315D, on rotten Picea fallen trunk, 27 Apr. 2010, Karasiński $5251 a$ (D.K.); 343D, on well-rotted Picea fallen trunk, 16 Aug. 2009, Karasiński 3849 (D.K.); 346D, on very rotten Picea fallen trunk, 13 Oct. 2009, Karasiński 4681 (D.K.); 370C, on well-rotted stump of deciduous tree, 10 Aug. 2009, Karasiński 3704 (D.K.); 374B, on rotten Acer platanoides fallen trunk, 15 Sept. 2009, Karasiński 4181 (D.K.). 
Literature REPORTs: Błoński et al. 1888 (as Polyporus mucidus), Domański 1965 (as Fibuloporia mollusca), Domański 1972b (as Fibuloporia donkii), Szczepkowski et al. 2008 (as Ceriporiopsis mucida), Niemelä 2013, Gierczyk et al. 2014 (as Ceriporiopsis mucida).

\section{Postia alni Niemelä \& Vampola}

Fig. 16E

Specimens examined: 260D, on Populus fallen trunk, 12 Oct. 2009, Karasiński 4622 (D.K.); 284B, on Carpinus fallen trunk, 15 Aug. 2009, Karasiński 3800 E (D.K.); 285C, on Corylus avellana fallen branch, $28 \mathrm{Apr}$. 2010, Karasińśki 5330 (D.K.); 370A, on Populus fallen trunk, 22 Sept. 2009, Karasiński 4393 (D.K.); 370C, on Populus fallen trunk, 22 Sept. 2009, Karasiński 4405 (D.K.); 385D (H.M.W. M-1367); 439D (H.M.W. M-2042); 500C (Podolany Reserve), on Corylus avellana fallen branch, 27 Sept. 2006, Karasiński 0609276035 (D.K.).

LITERATURE REPORTS: Jaroszewicz 1996 (as Tyromyces subcaesius), Szczepkowski et al. 2008 (as Oligoporus alni), Niemelä 2013, Gierczyk et al. 2014.

\section{Postia caesia (Schrad.) P. Karst.}

Fig. 16F

SPeCimens EXAMINED: 214D (Szczekotowo Reserve), on Picea fallen trunk, 17 Oct. 2014, Karasiński 11003 (D.K.); 375D (D.K. 10952); 413A (H.M.W. M-1889); 413D (Sacharewo), on Picea fallen trunk, 25 Sept. 1994, Wotkowycki (H.M.W. M-1888); 439A (H.M.W. M-0075); 462B (H.M.W. M-1745); 489 (in the vicinity of Nieznanowo Reserve), on Pinus stump, 24 Sept. 2006, Karasiński $060924-5609$ (D.K.); 572A (Michnówka Reserve), on Picea fallen trunk, 19 Sept. 2004, Wotkowycki (H.M.W. 3112); 730A (Starzyna Reserve), on Picea, 23 Sept. 2004, Wotkowycki (H.M.W. 3125); BNP, SE part, west of Dziedzinka house, on Picea big fallen tree, 12 Oct. 2008, Niemelä 8457 (KRA).

Literature Reports: Błoński 1889a (as Polyporus caesius), Bujakiewicz et al. 1992 (as Tyromyces caesius), Orłoś 1960, 1961 (as Leptoporus caesius), Domański 1967 (as Tyromyces caesius), Anonymous 1968 (as Tyromyces caesius), Bujakiewicz 1994 (as Tyromyces caesius), Skirgiełł 1997 (as Tyromyces caesius), Skirgiełło 1998 (as Postia caesia), Szczepkowski et al. 2008 (as Oligoporus caesius), Bujakiewicz
\& Kujawa 2010 (as Oligoporus caesius), Gierczyk et al. 2013, Niemelä 2013.

Postia ceriflua (Berk. \& M. A. Curtis) Jülich Fig. 16G

Specimens exAmined: 285C, on dead standing Pinus trunk, 21 Sept. 2010, Karasiński 5953 (D.K.); 288D, on Picea rotten stump, 22 Sept. 2011, Karasiński 6448 (D.K.); 369D, on rotten Pinus log, 16 Sept. 2009, Karasiński 3957 (D.K.); 369D, on rotten Pinus log, 13 Sept. 2011, Karasiński 6230 (D.K.); 373, on Quercus fallen trunk, 26 July 2009, Karasiński 3541 (D.K.); 374C, on Quercus fallen trunk, 10 Aug. 2009, Karasiński 3713 (D.K.); 374C, on Quercus fallen trunk, 21 Sept. 2011, Karasiński 6408 (D.K.); 374D, on rotten Pinus log, 23 Sept. 2010, Karasiński 6008 (D.K.); 374C, on Pinus fallen log, 21 Sept. 2011, Karasiński 6404 (D.K.).

LITERATURE REPORTS: Niemelä 2013.

REMARKs. According to Wojewoda (2003) this species was reported from Poland by Ryvarden and Gilbertson (1994) 'on map of Europe, without localities', but this is a mistake made by Wojewoda (see Ryvarden \& Gilbertson 1994: 407).

Postia floriformis (Quél.) Jülich

Fig. $16 \mathrm{H}$

Specimens examined: 254D, on roots of Picea fallen trunk, 12 Sept. 2011, Krasiński 6218 (D.K.); 412C, at base of Picea dead standing tree, 9 Sept. 2001, Wotkowycki (H.M.W. M-1576); BF, on Picea trunk, 18 Sept. 1960, Domański (KRAM F-SD 946, as Tyromyces floriformis); BF, on Picea trunk, 26 Aug. 1956, Domański (KRAM F-SD 535, Tyromyces floriformis); BF, on Picea trunk, 17 Sept. 1960, Domański (KRAM F-SD 947, Tyromyces floriformis); BF, on deciduous trunk, 5 Aug. 1962, Domański (KRAM F-SD 2245, Tyromyces floriformis); BF, on Tilia sp. trunk, 6 Aug. 1962, Domański (KRAM F-SD 2246, Tyromyces floriformis); BNP, Dyrekcyjny Tryb, on Picea fallen big trunk, 15 Sept. 2009, Niemelä 8655 (KRAM F-58161).

LITERATURE REPORTS: Domański et al. 1967 (as Tyromyces floriformis), Domański 1967 (as Tyromyces floriformis), Domański et al. 1973 (as Tyromyces floriformis), Niemelä 2013.

Fig. 16. A - Porodaedalea chrysoloma (Fr.) Fiasson \& Niemelä, B - Porodaedalea pini (Brot.) Murrill, C - Porotheleum fimbriatum (Pers.) Fr., D - Porpomyces mucidus (Pers.) Jülich, E - Postia alni (Pers.) Jülich, F - Postia caesia (Schrad.) P. Karst., G-Postia ceriflua (Berk. \& M. A. Curtis) Jülich, H - Postia floriformis (Quél.) Jülich. Photo D. Karasiński (A-H). 


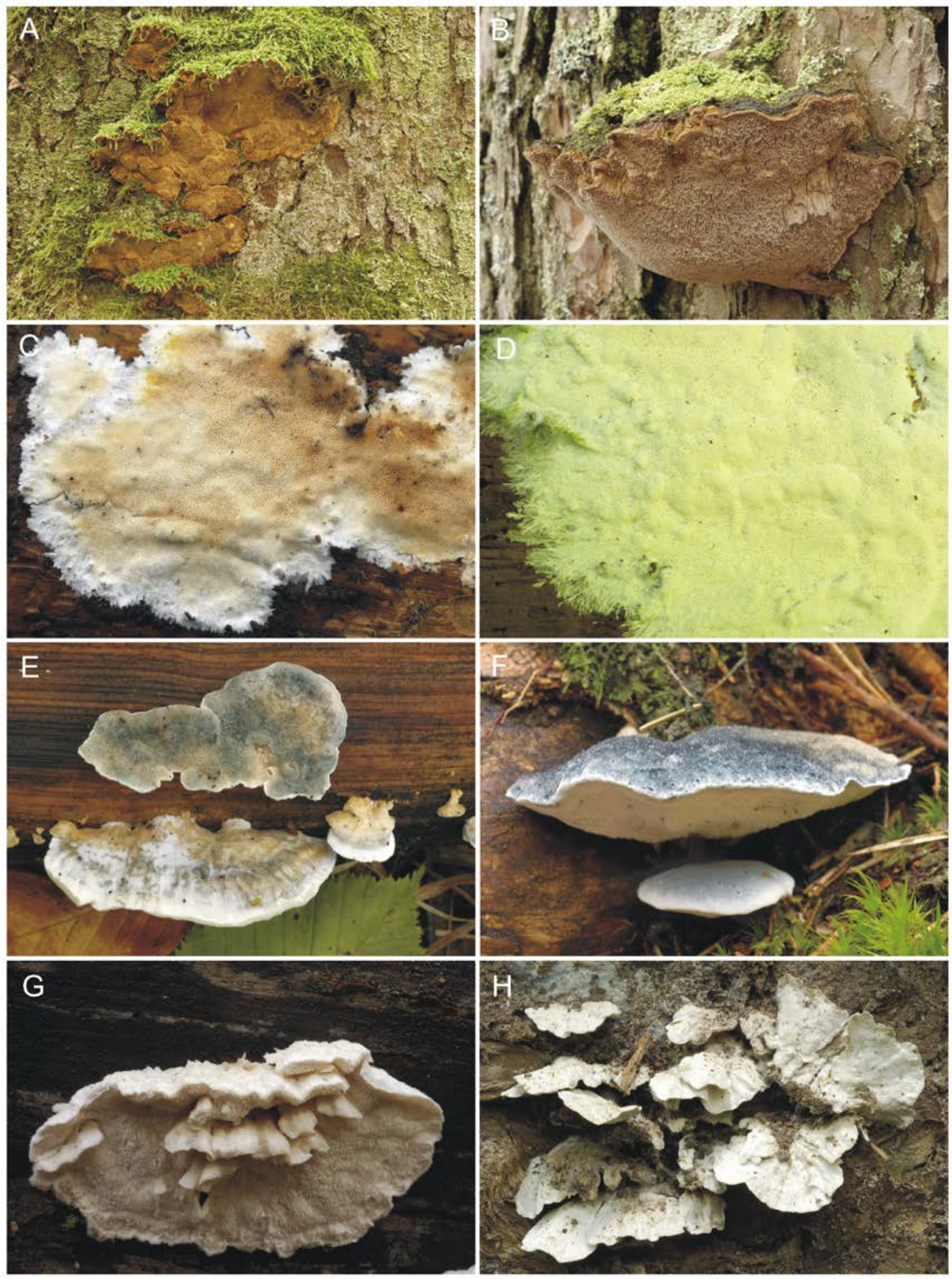


Postia folliculocystidiata (Kotl. \& Vampola)

Niemelä \& Vampola

Fig. 17A

Specimens examined: 373C, Picea fallen trunk, 24 Apr. 2009, Karasiński 2916 (D.K.).

Literature RePORTS: None. This species is new for Poland.

REMARKs. This is possibly a very rare species in Europe, characterized by having small pendent basidiomata attached to the substrate in one point, and large, pyriform, capitate or broadly clavate hymenial cystidia distinctly projecting above the basidia. According to Ryvarden and Melo (2014) Oligoporus folliculocystidiatus Kotl. \& Vampola (= Postia folliculocystidiata) and Leptoporus minusculoides Pilát ex Pilát [= Postia minusculoides (Pilát ex Pilát) Boulet] are synonyms of Postia ceriflua. However, on the basis of materials recently collected in the BF, all represent distinct species. See also Vampola et al. (2014).

\section{Postia fragilis (Fr.) Jülich}

Fig. 17B

SPeCimens EXAmined: 135C, on Picea fallen trunk, 8 July 2009, Karasiński 3339e (D.K.); 226A, on Picea fallen trunk, 30 July 2009, Karasiński 3680 (D.K.); 272 (Lipiny Reserve), on Picea stump, 26 Sept 2006, Karasiński 060926-5897 (D.K.); 374C, on Pinus log, 21 Aug. 2009, Karsiński 3994 (D.K.); 398C, on Picea fallen trunk, 18 Sept. 2009, Karasiński 4259 (D.K.); 442D (H.M.W. M-2140); 572A (H.M.W. M-3108); 698 (Starzyna Reserve), on Picea stump, 28 Sept. 2006, Karasiński 060928-6156 (D.K.).

LITERATURE REPORTS: Domański et al. 1967 (as Tyromyces fragilis), Domański 1967 (as Tyromyces fragilis), Anonymous 1968 (as Tyromyces fragilis), Domański et al. 1973 (as Tyromyces fragilis), Szczepkowski et al. 2008 (as Oligoporus fragilis), Niemelä 2013.

\section{Postia guttulata (Peck) Jülich Fig. 17C}

Specimens eXAmined: 105B (D.K. 3440); 111B (D.K. 3370C); 135C (D.K. 3342C); 135D, on Picea trunk, 8 July 2009, Karasiński 3340 (D.K.); 224B (D.K. 3948A); 224B (D.K. 3952A); 226A (D.K.3687F); 254D
(D.K. 3804B); 255D (D.K. 3653), 257D, on Picea stump, 21 Sept. 2009, Karasiński 4342 (D.K.); 261A (D.K. 3375), 285A, on Picea fallen trunk, 29 July 2009, Karasiński 3617D (D.K.); 285A (H.M.W. M-3660); 285C (D.K.5299C), 287C (D.K. 3779); 289C (D.K. 3746B); 315B (D.K. 3913E); 316B (D.K. 3789A); 318B (D.K. 3744G); 319C (D.K. 3614); 340A (D.K. 4227A); 340C (D.K. 4319); 342C (D.K. 4787B); 345A (D.K. 3718F); 374D (D.K. 4196A); 375A (D.K. 4279B); 402 (D.K. 3965B); 402B (H.M.W. M-3649); 417D (H.M.W. M-3109); 698 (Starzyna reserve), on Picea stump, 28 Sept. 2006, Karasiński 060928-6172 (D.K.).

LITERATURE REPORTS: Domański 1967 (as Tyromyces guttulatus), Anonymous 1968 (as Tyromyces stipticus f. guttulatus), Domański et al. 1973 (as Tyromyces guttulatus), Niemelä 1978a (as Tyromyces guttulatus), Szczepkowski et al. 2008 (as Oligoporus guttulatus), Karasiński et al. 2009, Szczepkowski et al. 2010 (as Oligoporus guttulatus), Gierczyk et al. 2013, Niemelä 2013, Gierczyk et al. 2014.

REMARKs. This species is rather common in the BF on stumps and fallen trunks of Picea. In Poland the species has not been so far recorded outside the BF (Wojewoda 2003).

Postia lactea (Fr.) P. Karst.

Fig. 17D

Specimens eXAmined: 226A, on Pinus log, 11 Oct. 2009, Karasiński 4573 (D.K.); 370, on Populus fallen log, 22 Sept. 2009, Karasiński 4404 (D.K.); 437A (Sacharewo), on fallen coniferous trunk, 26 Aug. 2003, Wotkowycki (H.M.W. M-3023).

Literature REPORTs: Błoński 1889a (as Polyporus lacteus), Pilát 1950 (as Leptoporus lacteus), Orłoś 1960, 1961 (as Leptoporus lacteus), Domański et al. 1967 (as Tyromyces lacteus), Kotlaba \& Lazebniček 1967 (as Tyromyces lacteus), Anonymous 1968 (as Tyromyces lacteus), Domański et al. 1973 (as Tyromyces lacteus), Bujakiewicz et al. 1992 (as Tyromyces lacteus), Skirgieło 1997 (as Tyromyces lacteus).

Remarks. Postia lactea is similar to P. tephroleuca (Fr.) Jülich and $P$. stiptica (Pers.) Jülich but differs in having context without the bitter taste that characterizes the latter species, and in

Fig. 17. A - Postia folliculocystidiata (Kotl. \& Vampola) Niemelä \& Vampola (dried specimen D.K. 2916), B - Postia fragilis (Fr.) Jülich, C - Postia guttulata (Peck) Jülich, D - Postia lactea (Fr.) P. Karst., E - Postia leucomallella (Murrill) Jülich, F Postia lowei (Pilát ex Pilát) Jülich, G - Postia luteocaesia (A. David) Jülich (photographed in Kampinos National Park, central Poland), H - Postia minusculoides (Pilát ex Pilát) Boulet. Photo D. Karasiński (A-H). 

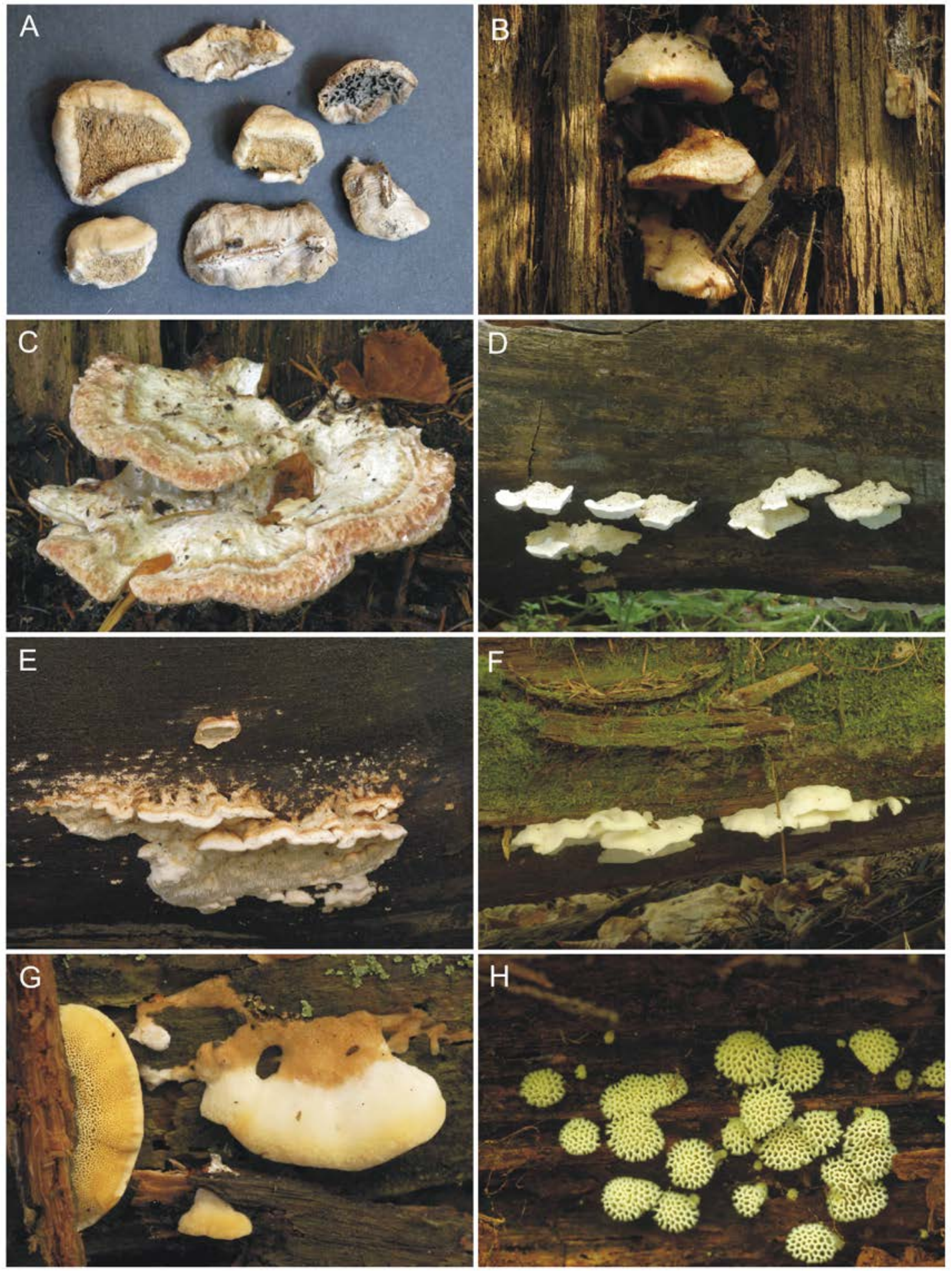
having a pure white pileal surface in contrast to the mouse-grey pileal surface of the former species. The hymenial parasite Tremella polyporina D. A. Reid was found inside the tubes of specimen Karasiński 4404.

\section{Postia lateritia Renvall}

Specimens examined: BF, on Picea, 28 Aug. 1956, Domański (KRAM F-SD 5374 as Tyromyces fragilis); 370C, Poprzeczny Tryb, east of 'Mogiłki' graves, on decorticate Picea fallen trunk, 9 Sept. 2009, Niemelä 8571 (KRAM F-58165).

LITERATURE REPORTS: Niemelä 2013.

Remarks. This species is similar to Postia fragilis and is easily confused with it. It was previously known only from Northern Europe (Fennoscandia) and one locality in Spain (Renvall 1992; Ryvarden \& Melo 2014). One specimen preserved in KRAMF-SD, originally identified by Domański as Tyromyces fragilis, very well fits the species concept of Postia lateritia. It has basidiospores 4.8-6.2 × 1.2-1.4 $\mu \mathrm{m}$, that is, more allantoid and narrower than in P. fragilis. The latter has basidiospores 1.6-2.2 $\mu \mathrm{m}$ wide. Another species similar to $P$. lateritia is $P$. leucomallella (Murrill) Jülich, which has almost identical basidiospores but forms hymenial gloeocystidia.

\section{Postia leucomallella (Murrill) Jülich Fig. 17E}

Specimens examined: 135C, on Pinus fallen trunk, 8 July 2009, Karasiński 3342b (D.K.); 284C, 340, on Pinus fallen log, 16 Sept. 2009, Karasiński 4229 (D.K.); 374, on Pinus fallen log, 26 July 2009, Karasiński 3542 (D.K.); 387 (W. Szafer Landscape Reserve), on Pinus log, 20 Sept. 2013, Karasiński 10099B (D.K.); 398B, on Picea, 31 Oct. 2001, Wotkowycki (H.M.W. M-2181); 698 (Starzyna Reserve), on Pinus trunk, Piatek s.n. (D.K. 6625).

LiTERATURE REPORTS: Anonymous 1968 (as Tyromyces gloeocystidiatus), Niemelä 2013.

Postia lowei (Pilát ex Pilát) Jülich

Fig. 17F

Specimens examined: 226A, on Picea fallen log, 20 Sept. 2011, Karasiński 6376 (D.K.); 257C, on Picea rotten trunk, 21 Sept. 2009, Karasiński 4331 (D.K.); 284D, on Picea fallen trunk, 13 Sept. 2011, Karasiński 6286 (D.K.); 316B, on Picea fallen trunk, 14 Aug. 2009,
Karasiński 3798 (D.K.); 375B, on Picea fallen trunk, 27 July 2009, Karasiński 3576 (D.K.); 375D, on well rotten Picea fallen trunk, 13 Oct. 2009, Karasiński 4669 (D.K.); 375D (D.K. 10962); 402A, on rotten Picea fallen trunk, 23 Sept. 2007, Karasiński 070923-8154 (D.K.).

LITERATURE REPORTS: Domański 1964a, 1965, Domański et al. 1967, Domański 1967, Kotlaba \& Lazebniček 1967, Anonymous 1968, Domański 1972b, Domański et al. 1973, Skirgiełło 1998, Niemelä 2013, Vampola et al. 2014. All literature reports (except Niemelä 2013; Vampola et al. 2014) published as Tyromyces lowei.

REMARKs. An excellent description of the species and an extensive discussion of the features differentiating it from all similar and related species are provided by Vampola et al. (2014).

Postia luteocaesia (A. David) Jülich Fig. 17G

LITERATURE REPORTS: Niemelä 2013.

REMARKs. This species was reported by Niemelä (2013) without a precise locality. We did not find it in the BF.

Postia minusculoides (Pilát ex Pilát) Boulet

Fig. $17 \mathrm{H}$

SPECIMENS EXAMINED: 374C, inside hollow of very rotten Picea fallen big log, 21 Aug. 2009, Karasiński 3997 (D.K.); 374C, in cavities on very rotten trunk of coniferous tree, 21 Sept. 2011, Karasiński 6412 (D.K.).

Literature Reports: None. This species is new for Poland.

REMARKS. This species is characterized by having minute pendent basidiomata, thin-walled hyphae in both context and tube trama, numerous slender fusoid cystidioles in the hymenium, and narrowly ellipsoidal to short-cylindrical basidiospores 4.2-5.2 × 2.4-2.6 $\mu \mathrm{m}$ (in specimens studied). Postia minusculoides is considered by some mycologists (e.g., Ryvarden \& Gilbertson 1994, Ryvarden \& Melo 2014) to be a synonym of P. ceriflua, which indeed has basidiospores similar in shape and size but differs by having larger and usually effused-reflexed or even pileate basidiomata, the absence of cystidioles in the hymenium, and the presence of thick-walled hyphae in the context and trama of tubes. 


\section{Postia stiptica (Pers.) Jülich}

Specimens examined: 314C, at base of Quercus(!) dead standing trunk, 13 Sept. 2011, Karasiński 6240 (D.K.); 370C, on Picea stump, 4 July 2009, Karasiński 3228 A (D.K.) 399, on Picea, 31 Aug. 1973, Wojewoda s.n. (KRAM F-58162); 486A (Nieznany Bór), on fallen trunk, 11 June 2003, Wotkowycki (H.M.W. M-3024); 697B (Starzyna Reserve), at base of Picea living tree, 28 Sept. 2006, Karasiński 060928-6166 (D.K.).

Literature RePORTs: Błoński et al. 1888 (as Polyporus albidus), Błoński 1889a (as Polyporus alutaceus), Domański 1960 (as Leptoporus albidus), Orłoś 1960, 1961 (as Leptoporus stipticus), Bujakiewicz 1994 (as Tyromyces stipticus), Domański et al. 1967 (as Tyromyces stipticus), Domański 1967 (as Tyromyces stypticus), Anonymous 1968 (as Tyromyces stipticus), Bujakiewicz et al. 1992 (as Tyromyces stipticus), Bujakiewicz 1994 (as Tyromyces stipticus), Skirgiełł 1997 (as Tyromyces stipticus), Szczepkowski et al. 2008 (as Oligoporus stipticus), Bujakiewicz \& Kujawa 2010 (as Oligoporus stipticus), Niemelä 2013, Gierczyk et al. 2014.

REMARKs. This species differs from similar species such as P. lactea and P. tephroleuca by having a strongly bitter taste and wider, slightly shorter basidiospores.

\section{Postia tephroleuca (Fr.) Jülich}

SPECIMENS EXAmined: 225D, on fallen trunk of deciduous tree (Quercus?), 20 Sept. 2011, Karasiński 6391 (D.K.); 369D, on Picea fallen trunk, 18 Sept. 2011, Karasiński 6330 (D.K.); 374D, on Pinus fallen log, 12 Aug. 2009, Karasiński 3731 (D.K.); 375A, on Picea fallen trunk, 19 Sept. 2009, Karasiński 4280 (D.K.); BNP, Masiewski Tryb, north of Dziedzinka hause, on Populus, 15 Oct. 2008, Niemelä 8500 (KRA).

LiteratURe REPORTS: Bujakiewicz et al. 1992, Skirgiełło 1997, Szczepkowski et al. 2008 (as Oligoporus tephroleucus), Niemelä 2013.

REMARKS. The specimens collected in the BF on coniferous wood differ from those on hardwoods by having a more strigose pileal surface and a thinner context.

Pycnoporellus alboluteus (Ellis \& Everh.) Kotl. \& Pouzar

Fig. 18A

Specimens eXAmined: 224B (D.K. 3940); 224D (D.K. 3932); 257D, on Picea fallen log, 21 Sept. 2009, Karasiński 4343 (D.K.); 284B (D.K. 3814F); 285A, on Picea fallen trunk, 29 July 2009, Karasiński 3617A (D.K.); 287C (D.K. 3778); 288D, on Picea fallen trunk, 12 Aug. 2009, Karasiński 3743B (D.K.); 314C (D.K. 5176); 316B (D.K. 3782); 317A (D.K. 5364A); 318B (D.K. 3744D); 318D (D.K. 3873); 318D (D.K. 3877); 319A (D.K. 3735A); 319C (D.K. 3597A); 319C (D.K. 3603); 319C (D.K. 3748I); 340F (D.K. 4231A); 340G (D.K. 4239); 346A (D.K. 3720); 346D (D.K. 3726); 370C (D.K. 3698A); 370C (D.K. 4739); 370D (D.K. 4758); 373C (D.K. 3472); 373C (D.K. 3478 albinotic form); 374A (D.K. 4170); 374C, on Picea fallen trunk, 11 July 2009, Karasiński 3398 (D.K.); 399B (D.K. 5063).

LiteratURe REPORTS: Domański 1959b (as Phaeolus alboluteus), Gumińska 1963 (as Phaeolus alboluteus), Domański 1965, 1967, Anonymous 1968, Domański 1972b, Niemelä 1980, Piątek 2002c, 2003, Niemelä 2005, Niemelä 2013.

REMARKS. This is one of the rarest poroid species in Europe, known from a few sites in the northern and central parts of the continent (Ryvarden $\&$ Melo 2014). It is associated with old-growth forests and dead wood of conifers. According to recent observations, $P$. alboluteus has a relatively large population in the $\mathrm{BF}$ but so far has been found only in the BNP area and never outside its borders. The species is characterized by having usually large basidiomata with an orange-yellow hymenial surface and pores larger than $1 \mathrm{~mm}$ in diameter, which finally form an irpicoid to dentate or even almost hydnoid hymenophore. In rare cases, specimens of this species may be almost white, but such an albinotic form was observed only once (specimen Karasiński 3478).

Pycnoporellus fulgens (Fr.) Donk Fig. 18B

SPECIMENS EXAMINED: 135C (D.K. 3343); 194D (D.K. 3669B); 225A (D.K. 4137); 225B (D.K. 4601); 225C (D.K. 3687B); 226A (D.K. 3349A); 226B (D.K. 3683A); 254D (D.K. 3808A); 255B (D.K. 3653A); 256A (D.K. 3644); 313A (D.K. 3509); 314A (D.K. 3303C); 315C (D.K. 3926); 315D (D.K. 3930A); 318D (D.K. 3880B); 319A (D.K. 3731A); 319C (D.K. 3748H); 319C (D.K. 3757E); 340C (3278B); 340F, on Picea stump + F. pinicola, 6 July 2009, Karasiński 3289 (D.K.); 340F (D.K. 4316E); 344A (D.K. 3837A); 346A (D.K. 3723A); 346D (D.K. 3581); 370C, on Picea stump and Fomitopsis pinicola dead basidiome, 4 July 2009, Karasiński 3228 (D.K.), 372B (D.K. 3986A); 399B (D.K. 3248A), 399D (D.K. 5070A); 697B (Starzyna 
Reserve), on Picea fallen log and Fomitopsis pinicola dead basidiome, 28 Sept. 2006, Karasiński 0609286191 (D.K.).

Literature REPORTs: Błoński et al. 1888 (as Polyporus lithuanicus), Błoński 1889b (as Ochroporus lithuanicus), Bendix 1967 (as Pycnoporellus fibrillosus), Anonymous 1968 (as Pycnoporellus fibrillosus), Domański et al. 1973, Skirgiełło 1997, Piątek 2002d, Niemelä 1980, Szczepkowski et al. 2008, 2010, Gierczyk et al. 2013, Niemelä 2013, Gierczyk et al. 2014.

\section{Pycnoporus cinnabarinus (Jacq.) P. Karst.}

Fig. 18C

SPECIMENS EXAMINED: 214D (Szczekotowo Reserve), on Carpinus fallen trunk, 17 Oct. 2014, Karasiński 11017 (D.K.); 256D (H.M.W. M-3416b); 285A (H.M.W. M-3416a).

LiterATURE REPORTS: Błoński et al. 1888 (as Trametes cinnabarinus), Błoński 1889a (as Polyporus cinnabarinus), Karpiński 1949 (as Trametes cinnabarina), Skirgiełło 1965, Niemelä 2013.

Rhodonia placenta (Fr.) Niemelä, K.-H. Larss. \& Schigel

Fig. 18D

Specimens examined: 194D (D.K. 3662); 224B (D.K. 3949); 226A (D.K. 3671); 226B (D.K. 3689); 256D (D.K. 3619); 261A (D.K. 3377, 3390); 284B, on decorticate Pinus fallen trunk, 7 July 2009, Karasiński 3310 (D.K.); 315B (D.K. 3916); 316B (D.K. 3795A); 316C (D.K. 5270); 318D (D.K. 3875); 319A, on Picea fallen trunk, 28 July 2009, Karasiński 3604 (D.K.); 340F (D.K. 4244); 343D (D.K. 3837B, 3848); 344A (D.K. 3718H); 370C (D.K. 3704B); 372B (D.K. 3991); 374D, on Pinus log, 26 July 2009, Karasiński 3547 (D.K.); 375B (D.K. 3588).

LiTERATURE REPORTS: Domański 1963, 1965, 1965b, 1967, 1970e (as Ceriporiopsis placenta), Domański 1972 b (as Poria placenta and P. placenta f. placenta), Gierczyk et al. 2013, Niemelä 2013.

REMARKs. The species is quite common in the study area on wood of conifers. The basidiomata of this species vary in color from pure white to vivid pink.
Rigidoporus crocatus (Pat.) Ryvarden Fig. 18E

SPECIMENS EXAMined: 130C (D.K. 4111); 194C (D.K. 3360A); 225A (D.K. 4129A), 252D (Pogorzelce reserve) (KRAM F-51549); 255D (D.K. 5226); 284B (D.K. 3814E); 285A (D.K. 5296); 313B (D.K. 3514A); 316B (D.K. 3792); 318B (D.K. 3744A); 318D (D.K. 3871A), 319C (D.K. 3757, 3759, 3767); 340B (D.K. 4197); 340F (D.K. 4241C); 340G (D.K. 4298); 341D, on Quercus fallen log, 17 Oct. 2009, Karasiński 4769 (D.K.); 346D (D.K. 4697); 369C (D.K. 4382); 373D (D.K. 3460); 375A (D.K. 4270); 375A on Picea fallen trunk, 16 Sept. 2009, Karasiński 4283 (D.K.); 375B (D.K. 4283); 399C (D.K. 5003, 5045); 400B, on Carpinus fallen trunk, 5 July 2009, Karasiński 3265A (D.K.); 402A (D.K. 4966); 488C (Nieznanowo Reserve), on rotten trunk, 13 Sept. 2005, Karasińśki 050913-7638 (D.K.); 697B (Starzyna Reserve), on Betula fallen log, 28 Sept. 2006, Karasiński 060928-6212 (D.K.).

Literature RePORTS: Domański 1959a (as Poria nigrescens), Domański 1962, 1965, 1967 (as Podoporia nigrescens), Kotlaba \& Lazebniček 1967 (as Rigidoporus nigrescens), Anonymous 1968 (as Podoporia nigrescens), Domański 1972b (as Rigidoporus nigrescens), Szczepkowski et al. 2008, 2011, Niemelä 2013, Gierczyk et al. 2014.

REMARKS. This species is characterized by having resupinate, perennial basidiomata with a pinkish brown to dull brick-red hymenial surface and rigid consistency, a monomitic hyphal system with clampless hyphae forming dense structures in all parts of the basidiome, the absence of cystidia and the presence of subglobose basidiospores. It is quite frequently observed in the BF, especially in BNP, on both hardwoods and conifers (Betula, Carpinus, Fraxinus, Quercus, Picea, once on Pinus). In southern Poland it also occurs on Abies alba Mill.

Rigidoporus undatus (Pers.) Donk Fig. 18F

SPECIMENS EXAMINED: $\mathbf{2 5 6}$, on bark at base of Quercus living tree, 21 Sept. 2009, Karasiński 4335 (D.K.); 258C, on Fraxinus log, 21 Sept. 2009, Karasiński 4362 (D.K.); 340A, on Tilia fallen log, 22 Sept. 2010, Karasiński 5960 (D.K.).

Fig. 18. A - Pycnoporellus alboluteus (Ellis \& Everh.) Kotl. \& Pouzar, B - Pycnoporellus fulgens (Fr.) Donk, C - Pycnoporus cinnabarinus (Jacq.) P. Karst., D - Rhodonia placenta (Fr.) Niemelä, K. H. Larss. \& Schigel, E - Rigidoporus crocatus (Pat.) Ryvarden, F - Rigidoporus undatus (Pers.) Donk, G - Sarcoporia polyspora P. Karst., H - Schizopora paradoxa (Schrad.) Donk. Photo D. Karasiński (A-H). 

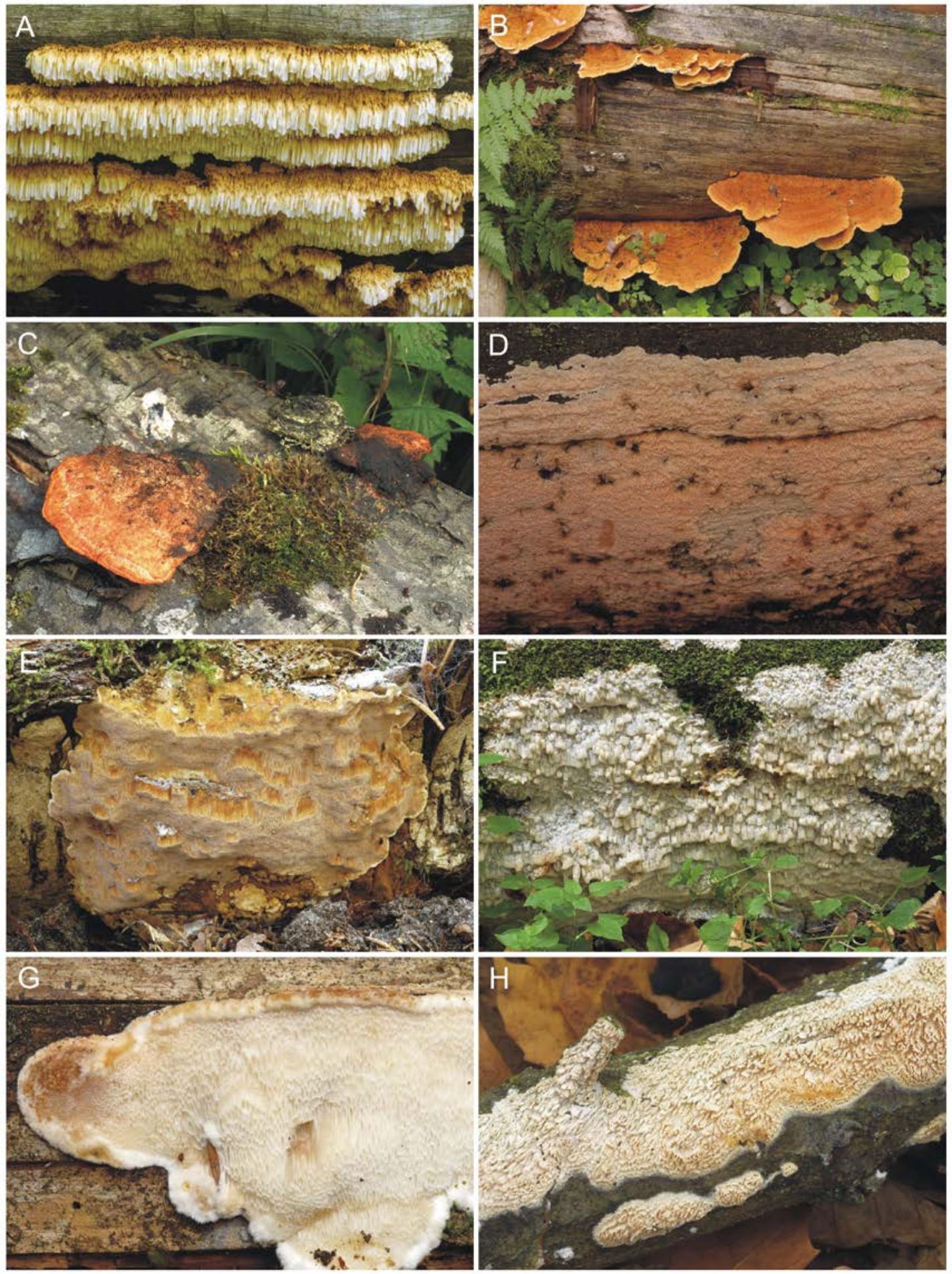
LITERATURE REPORTS: Niemelä 2013.

Remarks. This species is similar to Physisporinus vitreus but differs by having more robust basidiomata with smaller pores (6-8 per mm vs. 4-6 in P. vitreus), slightly smaller basidiospores and the presence of thin to thick-walled cystidia of tramal origin, apically encrusted, embedded in the trama and not projecting.

\section{Sarcoporia polyspora $\mathrm{P}$. Karst.}

Fig. $18 \mathrm{G}$

SPECIMENS EXAMINED: 224B, on rotten Picea fallen trunk, 19 Aug. 2009, Karasiński 3941 (D.K.); 253C, on Picea fallen trunk, 13 Sept. 2009, Karasiński 4094 (D.K.); 255, on Picea fallen log, 19 Sept. 2011, Karasiński 6348 (D.K.); 318D, on Pinus fallen log, 17 Aug. 2009, Karasiński 3866 (D.K.); 340F, on Pinus fallen trunk, 16 Sept. 2009, Karasiński 4230; 340G, on rotten Picea fallen log, 20 Sept. 2009, Karasiński 4289 (D.K.); 373C, on Picea fallen trunk, 13 July 2009, Karasiński 3469 (D.K.); 374B (D.K. 10944); 374C, on Picea fallen trunk, 15 Sept. 2009, Karasiński 4196 (D.K.); 375B (D.K. 10955).

LiterATURe REPORTs: Domański 1965, 1967 (as Tyromyces subcartilagineus), Kotlaba \& Lazebniček 1967 (as Parmastomyces kravtzevianus), Anonymous 1968 (as Tyromyces subcartilagineus), Domański 1972b (as Parmastomyces kravtzevianus), Szczepkowski et al. 2010 (as Parmastomyces transmutans), Niemelä 2013.

Schizopora flavipora (Berk. \& M. A. Curtis ex Cooke) Ryvarden

SPeCimens EXAMINED: 98B (D.K. 10978); 256D, on Alnus fallen trunk, 21 Sept. 2009, Karasiński 4329 (D.K.); 342C, on Carpinus fallen trunk, 17 Oct.2009, Karasiński 4773 (D.K.); 369, on very rotten deciduous trunk, 21 Sept. 2007, Karasiński 070921-2 (D.K.); 373, on bark of Picea fallen trunk, 27 June 2008, Karasiński 1785 (D.K.); 399A, on Carpinus fallen trunk, 2 Nov. 2009, Karasiński 5074 (D.K.).

LITERATURE REPORTS: Domański 1969c (as Schizopora phellinoides), Bujakiewicz et al. 1992 (as Schizopora carneo-lutea), Gierczyk et al. 2013, Niemelä 2013 (as Hyphodontia flavipora), Gierczyk et al. 2014.

Schizopora paradoxa (Schrad.) Donk Fig. 18H

SPECIMENS EXAMINED: 98B, on Carpinus fallen branch, 17 Oct. 2014, Karasiński 10982 (D.K.); 214D (D.K. 10999); 255D, on fallen branch (Quercus?), 26 Apr.
2010, Karasiński 5221 (D.K.); 372C, on Carpinus fallen trunk, 5 July 2009, Karasiński 3252F (D.K.); 385C, on Carpinus fallen branch, 8 Oct. 1998, Wotkowycki (H.M.W. M-1232); 399, on fallen branches of deciduous tree, 31 Aug. 1973, Wojewoda s.n. (KRAM F-58163); 413A (H.M.W. M-1249); 438A (H.M.W. M-1277).

LiteRATURE REPORTS: Błoński et al. 1888 (as Irpex paradoxus), Błoński 1889a (as Sistotrema paradoxum), Orłoś 1961 (as Poria versipora and Irpex obliquus), Domański 1965 (as Xylodon versiporus f. paradoxus), Domański 1967 (as Xylodon versiporus), Anonymous 1968 (as Xylodon versiporus), Domański 1969b, 1972b, Bujakiewicz et al. 1992, Bujakiewicz 1994, Skirgiełło 1997, 1998, Szczepkowski et al. 2008, Bujakiewicz \& Kujawa 2010, Gierczyk et al. 2013, Niemelä 2013 (as Hyphodontia paradoxa), Gierczyk et al. 2014.

Schizopora radula (Pers.) Hallenb.

SPECIMENS EXAMINED: 369A, on Alnus fallen branch, 10 March. 2002, Wotkowycki (H.M.W. M-2910); 374C, on Quercus robur fallen log, 23 Sept. 2010, Karasiński 6016 (D.K.); 374D, on Quercus log, 23 Sept. 2010, Karasiński 6021 (D.K.).

Literature RePORTS: Błoński 1889a (as Polyporus radula), Domański 1965 (as Chaetoporus radulus), Niemelä 2013 (as Hyphodontia radula), Gierczyk et al. 2014.

Sidera lenis (P. Karst.) Miettinen

Fig. 19A

Specimens examined: 399D, on Picea fallen trunk, 1 Nov. 2009, Karasiński 5033 (D.K.); 402C, on Picea fallen trunk, 22 Apr. 2009, Karasiński 2861 (D.K.).

Literature RePorts: Domański 1962 (as Poria lenis), Domański 1965, 1967 (as Amyloporia lenis), Anonymous 1968 (as Amyloporia lenis), Domański 1972 b (as Poria lenis and P. lenis var. radicata), Niemelä 2013.

REMARKs. The materials of Domański deposited in KRAM F-SD were not revised; they need to be re-examined to determine which of the two European poroid Sidera species were reported in the above-mentioned literature under the names Poria lenis and Amyloporia lenis.

Sidera vulgaris (Fr.) Miettinen

Fig. 19B

SPECIMENS EXAMINED: 224B, on Pinus fallen branch, 19 Aug. 2009, Karasiński 3942, 3950 (D.K.); 226A, on Pinus fallen log, 9 July 2009, Karasiński 3348 (D.K.); 
282D, on Picea fallen trunk, 25 July 2009, Karasiński 3526 (D.K.); 374D, on Pinus trunk, 24 Sept. 2011, Karasiński 6566 (D.K.); 375, on Picea fallen trunk, 13 Oct. 2009, Karasiński 4658 (D.K.); 375D (D.K. 10951); 399A, on Picea rotten log, 15 Oct. 2009, Karasiński $4716 a$ (D.K.); 399D, on Populus fallen trunk, 2 Nov. 2009, Karasiński 5051 (D.K.); 402A, on Populus fallen log, 31 Oct. 2009, Karasiński 4968 (D.K.); 402C, on rotten Picea fallen trunk, 25 June 2008, Karasiński 1745, 402C, on rotten Picea fallen trunk, 27 June 2008, Karasiński 1799 (D.K.); 415D, in the vicinity of Głęboki Kąt Reserve, on Picea fallen trunk, 22 May 2006, Karasiński 060522-5330 (D.K.).

LITERATURE REPORTS: Niemelä \& Dai 1997 (as Skeletocutis vulgaris), Niemelä 2013.

REMARKS. This species differs from $S$. lenis by having capitate hyphal ends on dissepiment edges, and smaller basidiospores 3-4 $\mu \mathrm{m}$ long (4-5 $\mu \mathrm{m}$ long in S. lenis).

\section{Skeletocutis amorpha (Fr.) Kotl. \& Pouzar}

SPeCimens eXamined: 135D, on Picea fallen trunk, 8 July 2009, Karasiński 3329 (D.K.); 385C (H.M.W. M-1233); 385D (Sacharewo), on Pinus stump, 10 Feb. 2002, Wolkowycki (H.M.W. M-2704); 438A (H.M.W. M-1300); 466D (H.M.W. M-2101).

Literature RePORTs: Błoński et al. 1888 (as Polyporus amorphus), Pilát 1950 (as Gloeoporus amorphus), Domański 1965 (as Skeletocutis amorpha f. resupinata), Domański 1967, Domański et al. 1967, 1973, Szczepkowski et al. 2008, Niemelä 2013, Gierczyk et al. 2014.

\section{Skeletocutis biguttulata (Romell) Niemelä}

Fig. 19C

Specimens EXAmined: 226A, on rotten trunk, 9 July 2009, Karasiński 3353 (D.K.); 226A, on Picea fallen trunk, 11 Oct. 2009, Karasiński 4580 (D.K.); 255D, on Picea trunk, 26 Apr. 2010, Karasiński 5218C (D.K.); 285A, on Picea fallen branch, 28 Apr. 2010, Karasiński 5289 (D.K.); 313B, on Picea fallen trunk, 25 July 2009, Karasiński 3515 (D.K.); 319, on Pinus fallen branch, 13 Aug. 2009, Karasiński 3762 (D.K.); 373D, on Picea log, 13 July 2009, Karasiński 3449 (D.K.).

Literature REPORTS: Piątek \& Cabała 2005, Niemelä 2013.

REMARKs. Possibly it was also reported previously by Domański (1972b) as Incrustoporia subincarnata. The herbarium specimens deposited in KRAM F-SD were revised by Piątek and Cabała (2005), and all of them represent $S$. biguttulata.

\section{Skeletocutis brevispora Niemelä}

Fig. 19D

Specimens examined: 319C, on Picea fallen trunk and Phellinidium ferrugineofuscum dead basidiome, 28 July 2009, Karasiński 3607 A (D.K.); 340B, on Picea fallen trunk and P. ferruginefuscum dead basidiome, 20 Sept. 2009, Karasiński 4305 (D.K.); 370C, on Picea fallen trunk and $P$. ferrugineofuscum dead basidiome, 10 Aug. 2009, Karasiński 3703E (D.K.); 374D, on wood of Picea fallen log close to and on dead basidiome of P. ferrugineofuscum, 26 July 2009, Karasiński 3534 (D.K.).

\section{LITERATURE REPORTS: Niemelä 2013.}

REMARKS. This species is a successor on wood previously decayed by Phellinidium ferrugineofuscum, often growing directly on its dead basidiomata. Microscopically the species is characterized by having short allantoid and fairly thick basidiospores $3.2-4.6 \times 1.4-1.8 \mu \mathrm{m}$ (in specimens examined) tapering towards the apiculus, without guttules, and having rather straight, sparsely encrusted hyphal ends at dissepiment edges.

\section{Skeletocutis carneogrisea A. David}

Specimens examined: 285C, on Picea fallen trunk and Trichaptum dead basidiome, 28 Apr. 2010, Karasiński 5291 (D.K.); 369C, on Picea fallen trunk and Trichaptum abietinum dead basidiomata, 22 Sept. 2009, Karasiński 4383 (D.K.); 439A, on Picea fallen trunk and Trichaptum dead basidiomata, 10 Dec. 2000, Wotkowycki (H.M.W. M-1454).

LiTERATURE REPORTS: Domański et al. 1967 (as Skeletocutis amorpha f. mollusca), Niemelä 2013, Gierczyk et al. 2014.

REMARKS. This species is similar to Skeletocutis amorpha but differs by having whitish or slightly greyish tubes in contrast to the yellow-orange or pinkish tubes of the latter species. Skeletocutis carneogrisea is a successor species; its predecessors are species of Trichaptum Murrill.

Skeletocutis chrysella Niemelä

Fig. 19E

SPeCimens EXAmined: 158, on bark of Picea fallen trunk close to Porodaedalea chrysoloma, 18 Sept. 2008, Karasiński 080918-1582 (D.K.); 373, on Picea fallen 
trunk close to P. chrysoloma, 27 June 2008, Karasiński 1776 (D.K.); 375, on bark of Picea fallen trunk close to P. chrysoloma, 24 Sept. 2010, Karasiński 6050 (D.K.); 402, on Picea trunk, close to P. chrysoloma, 31 Oct. 2009, Karasiński 4997 (D.K.).

Literature RePORTs: None. This species is new for Poland.

REMARKS. This is a successor species on spruce wood previously inhabited by Porodaedalea chrysoloma.

\section{Skeletocutis jelicii Tortič \& A. David Fig. 19F}

SPeCimens EXAmined: 346C, on rotten Picea fallen trunk, 21 Sept. 2011, Karasiński 6435 (D.K.); 369C, on very rotten Picea fallen trunk, 22 Sept. 2009, Karasiński 4377 (D.K.); 285C, on very rotten Picea fallen trunk, 20 Sept. 2010, Karasiński 5945 (D.K.).

LITERATURE REPORTS: Niemelä 2013.

REMARKS. This species differs from other species of Skeletocutis Kotl. \& Pouzar by having a monomitic hyphal system, ellipsoidal basidiospores (allantoid in other species), and the absence of encrusted hyphal ends on dissepiment edges. The species has a very thin basidiome with small pores 7-9 per mm, a hymenial surface with depressions after guttation droplets, and brown spots when mature. In the hymenium are encrusted hyphidia. Skeletocutis jelicii is a rare species previously known from the former Yugoslavia and Finland (Ryvarden \& Melo 2014).

\section{Skeletocutis kuehneri A. David}

Fig. 19G

Specimens eXamined: 373C, on Pinus fallen log and dead Trichaptum sp., 13 July 2009, Karasiński 3469 (D.K.); 373D, on Picea fallen log (coexist with Phellopilus nigrolimitatus), 13 July 2009, Karasiński 3449 (D.K.); 399C, on bark of Picea big fallen log close to Trichaptum abietinum dead basidiomata, 4 July 2009, Karasiński 3241 (D.K.); BNP, NE of the main gate, on Picea fallen tree, 10 Oct. 2008, Niemelä 8431 (KRA).

LITERATURE REPORTS: Niemelä 2013.

REMARKS. This species was previously reported from Poland by Piątek and Cabała (2005). The shape and size of basidiospores are the same in S. kuehneri, S. chrysella and S. stellae (Pilát) Jean Keller: allantoid, ca 3-4×0.7-1 $\mu \mathrm{m}$, without guttules. The latter has perennial basidiomata and small pores 7-10 per mm, while in the annual S. kuehneri and S. chrysella the pores are larger, ca 4-6 per mm. Skeletocutis kuehneri is a successor on wood previously inhabited by Trichaptum spp. and has a dimitic hyphal structure, unlike $S$. chrysella which grows together with Poradaedalea chrysoloma and produces binding hyphae in the subiculum.

\section{Skeletocutis nivea (Jungh.) Jean Keller}

Specimens examined: 214D (Szczekotowo Reserve), on Fraxinus fallen trunk, 17 Oct. 2014, Karasiński 11008 (D.K.); 252B (H.M.W. M-1530); 340C, on Fraxinus fallen branch, 6 July 2009, Karasiński 3276C (D.K.); 345A, on Corylus avellana fallen branch, 29 July 2009, Karasiński 3596 (D.K.); 375D (D.K. 10950); 384D (H.M.W. M-1647); 398D (H.M.W. M-1900); 412A (H.M.W. M-1347); 414D (H.M.W. M-2184); 438B (H.M.W. M-1413); 439B (Głęboki Kąt Reserve), on Fraxinus fallen twig, 22 March 2002, Wotkowycki (H.M.W. M-2702); 464D (H.M.W. M-1617); 486D (H.M.W. M-1898); 488C (H.M.W. M-1536).

LiTERATURE REPORTS: Domański et al. 1967 (as Tyromyces semipileatus), Domański 1967 (as Tyromyces semipileatus), Domański et al. 1973 (as Leptotrimitus semipileatus), Bujakiewicz et al. 1992, Bujakiewicz 1994, Skirgiełło 1997, Bujakiewicz \& Kujawa 2010, Niemelä 2013, Gierczyk et al. 2014.

\section{Skeletocutis odora (Sacc.) Ginns}

Fig. $19 \mathrm{H}$

SPECIMENS EXAMINED: 130C (D.K. 4121); 194D (D.K. 3666); 226A (D.K. 3673, DK 3676); 314D (D.K. 3931); 315B (D.K. 3795, DK 3912, DK 3917); 318D, on Betula fallen trunk, 17 Aug. 2009, Karasiński $3877 A$ (D.K.); 318D (D.K. 3860); 319C, on Picea trunk, 13 Aug. 2009, Karasiński 3760B (D.K.); 319C (D.K. 3754E); 340C (D.K. 3287); 340F (D.K. 4310, DK 3287); 343C (D.K. 3847); 344B (D.K. 3829); 345A

Fig. 19. A - Sidera lenis (P. Karst.) Miettinen, B - Sidera vulgaris (Fr.) Miettinen, C - Skeletocutis biguttulata (Romell) Niemelä, D - Skeletocutis brevispora Niemelä, E - Skeletocutis chrysella Niemelä, F - Skeletocutis jelicii Tortič \& A. David, G - Skeletocutis kuehneri A. David, H - Skeletocutis odora (Sacc.) Ginns. Photo D. Karasiński (A-H). 

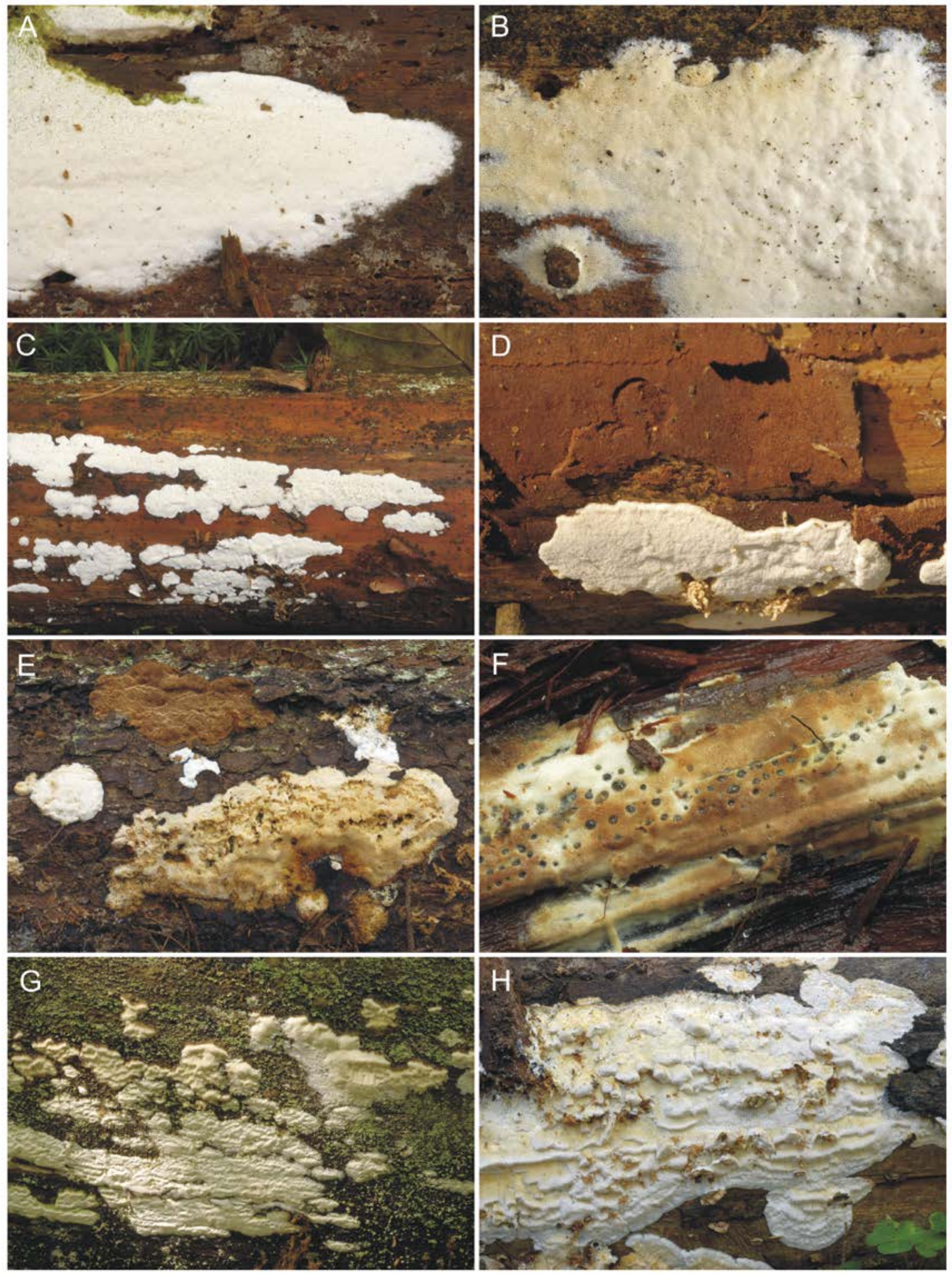
(D.K. 3716D); 346 (D.K. 4691); 370A (D.K. 4406); 370C (D.K. 3710); 374, on Pinus fallen log, coexisting with Antrodia infirma, 26 July 2009, Karasiński 3553A (D.K.); 402A, on Populus fallen trunk, 26 July 2009, Karasiński 3528 (D.K.).

LiTERATURE REPORTS: Domański 1962 (as Ceriporia gilvella), Domański 1965, 1967, 1969e, Domański 1972b (as Incrustoporia tschulymica), Piątek 2005c, Niemelä 2013, Gierczyk et al. 2014.

REMARKS. This species occurs frequently in the study area, mostly on Picea fallen trunks, but during recent observations was also recorded on Pinus, Betula and Populus. It is characterized by having annual, resupinate basidiomata, usually with an undulate hymenial surface, tubes up to $1 \mathrm{~cm}$ long, relatively large pores $2-5$ per $\mathrm{mm}$, a partly gelatinous context when fresh and a distinct smell similar to young garlic but sweeter. The hyphal ends at dissepiment edges are strongly encrusted and clearly flexuous or even spirally twisted. The size of basidiospores is intermediate for the genus, $4.0-5.5 \times 1.2-1.6 \mu \mathrm{m}$, and they lack guttules.

\section{Skeletocutis papyracea A. David}

Fig. 20A

Specimens EXAmined: 256, on Picea log, 29 July 2009, Karasiński 3639 (D.K.); 257D, on fallen log of Picea, 21 Sept. 2009, Karasiński 4346 (D.K.) 257D (D.K. 4346); 285A (D.K. 5288A); 319C (D.K. 3605, DK 3749); 340F (D.K. 3283); 369A, on rotten Picea fallen trunk, 14 July 2009, Karasiński 3482 (D.K.); 373C (D.K. 3456, 3965); 375B (D.K. 3569); 375, on Pinus fallen trunk, 24 Sept. 2010, Karasiński 6052 (D.K.).

Literature RePORTS: None. This species is new for Poland.

REMARKS. It is characterized by having rather small, very thin and hygrophanous basidiomata with minutely fimbriate margins and pores 4-5 per $\mathrm{mm}$, straight and sparsely encrusted hyphal ends on dissepiment edges, and cylindrical, slightly curved basidiospores 4-5.5 × 1.4-1.8 $\mu \mathrm{m}$, often with 1-2 guttules. Skeletocutis biguttulata has similar gut- tulate but longer basidiospores $(4.5-7.0 \mu \mathrm{m})$, but it produces spirally winding and strongly encrusted hyphae at dissepiment edges, and its basidiomata are larger, thicker and not hygrophanous.

Skeletocutis stellae (Pilát) Jean Keller Fig. 20B

SPeCimens EXAMINED: 130C (D.K. 4121A); 224B (D.K. 3942); 226C, on Picea fallen trunk, 30 July 2009, Karasiński 3682 (D.K.); 253C (D.K. 4089); 254D (D.K. 3813); 257C (D.K. 4338); 285A (D.K. 5307); 316B (D.K. 3789); 316C (D.K. 5259); 319B, on Pinus log, 14 Aug. 2009, Karasiński 3789 (D.K.); 340B (D.K. 4303); 344D (D.K. 3593); 370C (D.K. 3709); 373D (D.K. 4996A); 373D, on Picea big fallen log, 13 July 2009, Karasiński 3447 (D.K.); 374C (D.K. 3546); 374D (D.K. 4161); 375A (D.K. 4276); 399B (D.K. 3298E); 399C (D.K. 5048); 442B (W. Szafer Landscape Reserve), on very rotten fragment of fallen trunk, 23 June 2008, Karasiński 1711 (D.K.).

Literature REPORTS: Domański 1959a (as Poria stellae), Domański 1963, 1965, 1967, 1972b (as Incrustoporia stellae), Karasiński et al. 2009, Niemelä 2013.

REMARKS. It is characterized by having perennial, often large basidiomata, small pores 7-10 per $\mathrm{mm}$, allantoid basidiospores $3-4 \times 0.8-1 \mu \mathrm{m}$, the absence of guttules, and moderately winding hyphal ends at dissepiment edges with rather sparse encrustation.

Spongipellis delectans (Peck) Murrill Fig. 20C

LiTERATURE REPORTS: Orłoś 1960, 1961 (as Leptoporus bredecelensis), Domański et al. 1967 (as Spongipellis bredecelensis), Domański 1967 (as Spongipellis bredecelensis), Domański et al. 1973, Piątek et al. 2004, Tomšovský 2012, Niemelä 2013.

REMARKs. Possibly this species is very rare in the study area; we did not not confirm it in recent fieldwork; nor was it found among the specimens preserved in KRAM F-SD and identified by Domański as Spongipellis delectans. One specimen labelled with this name is a sterile basidiome of Trametopsis cervina (Schwein.) Tomšovsky

Fig. 20. A - Skeletocutis papyracea A. David, B - Skeletocutis stellae (Pilát) Jean Keller, C - Spongipellis delectans (Peck) Murrill (photographed in Puszcza Bukowa Forest, NW Poland), D - Spongipellis spumeus (Sowerby) Pat., E - Spongiporus rhodophilus Spirin \& Zmitr., F - Trametes gibbosa (Pers.) Fr., G - Trametes ochracea (Pers.) Gilb. \& Ryvarden, H - Trametes pubescens (Schumach.) Pilát. Photo D. Karasiński (A, B, D-H) and G. Domian (C). 

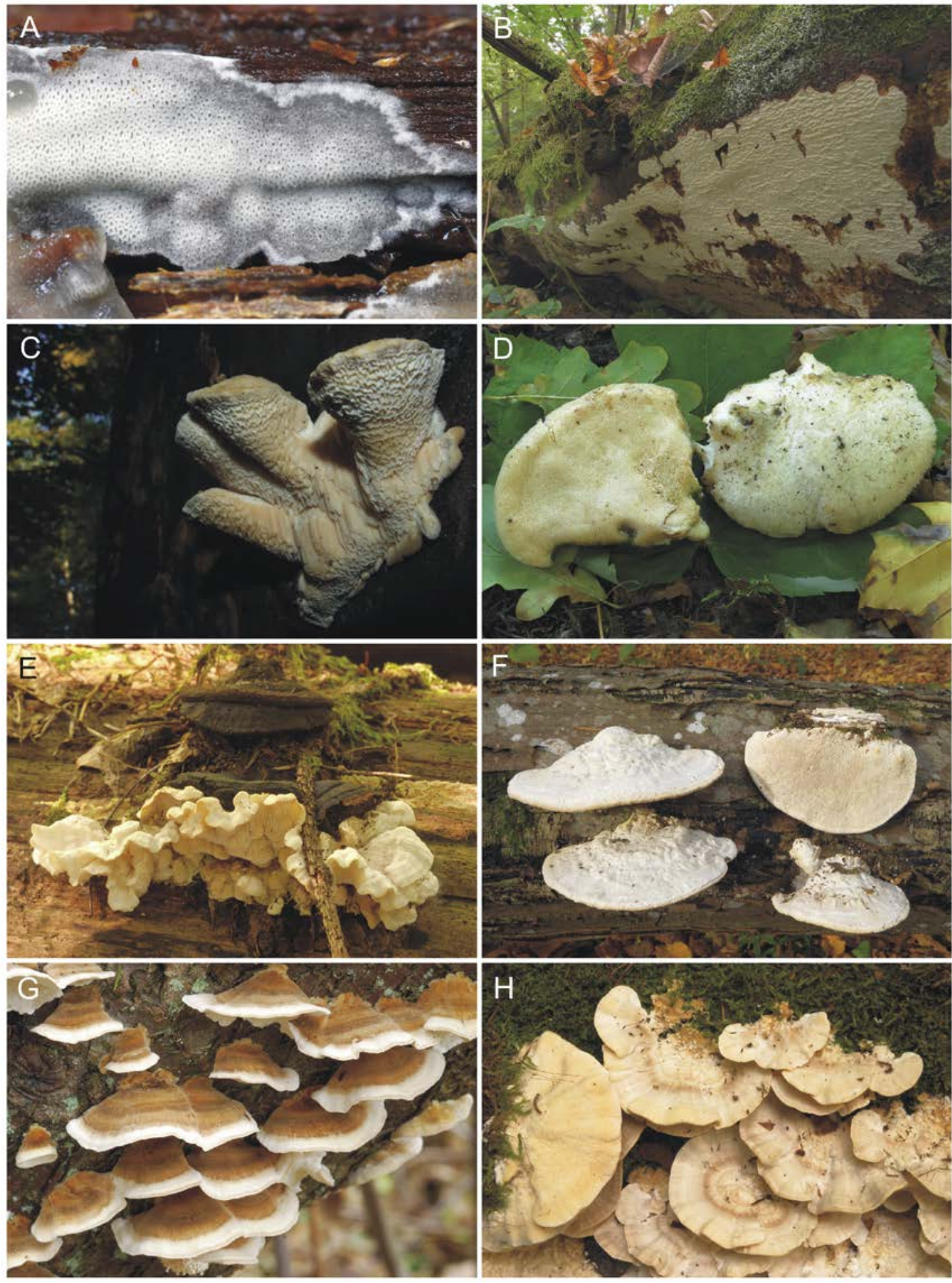
(on Carpinus, 20 Oct. 1963, Domański, KRAM F-SD 3442 as $S$. delectans). The second collection (KRAM F-SD 546), reported from BNP as S. delectans (Piątek et al. 2004), does not belong to this species either. This specimen was originally identified as Spongipellis litschaueri Lohwag, and that is indeed the correct name for this specimen. A specimen collected by Orłoś on 20 September 1957 (see Domański et al. 1973: tab. VIII, 2.) may be Spongipellis delectans. Herbarium material is stored in WAM (not studied). Tomšovský (2012) reported $S$. delectans from the BF based on a collection from 1955 deposited in PRM 884226. Niemelä (2013) reported this species based on literature data (after Domański et al. 1973).

\section{Spongipellis litschaueri Lohwag}

Specimens eXAmined: BF, on deciduous log (Quercus?), 25 Oct. 1959, Domański (KRAM F-SD 546).

LiteratURe RePORTS: Piątek et al. 2004 (as Spongipellis delectans).

REMARKS. It seems to be a very rare species in the study area, and was not confirmed during recent fieldwork. The species was earlier synonymized with Spongipellis delectans, and under this name the specimen cited above was published by Piątek et al. (2004). Recent molecular studies showed that Spongipellis litschaueri is a distinct species (Tomšovský 2012) which, besides its genetic divergence, differs in its ecology and macromorphology from $S$. delectans. It grows almost exclusively on Quercus (S. delectans predominatly on Fagus) and has thick basidiomata with a pileal context up to $7 \mathrm{~cm}$ thick (vs. up to $2 \mathrm{~cm}$ thick in S. delectans), the basidiome triquetrous in section, pores with thin dissepiments (thick in S. delectans) and basidiospores longer than $7 \mu \mathrm{m}$ on average (up to $7 \mu \mathrm{m}$ in $S$. delectans). In the specimen examined (KRAM F-SD 546), basidiospore size was $(6.0-) 6.2-7.6(-8.0) \times(4.6-) 4.8-6.2(-6.4) \mu \mathrm{m}$.

\section{Spongipellis spumeus (Sowerby) Pat. Fig. 20D}

SPeCimens EXAmined: 340B, on dead standing deciduous trunk, 24 Sept. 2010, Rutkowski s.n. (D.K. 6066).
LiTERATURE REPORTS: Domański et al. 1967, 1973, Piątek et al. 2004, Niemelä 2013 (as Spongiporus spumea), Gierczyk et al. 2013.

REMARKS. This species appears to be very rare in the study area. It differs from $S$. litschaueri and $S$. delectans mostly by having an almost even hymenial surface and smaller, regular pores (larger, irregular, daedaleoid, dentate and lacerate in S. delectans and S. litschaueri).

Spongiporus rhodophilus Spirin \& Zmitr.

Fig. 20E

SPeCIMENS EXAMINED: 135C, on rotten Picea fallen trunk, 8 July 2009, Karasiński 3328a (D.K.); 226A, on Populus fallen trunk, 9 July 2009, Karasiński 3346 (D.K.); 256A, on rotten Picea fallen trunk, 29 July 2009, Karasiński 3643 (D.K.); 284B, on rotten Picea fallen trunk, 15 Aug. 2009, Karasiński 3814d (D.K.); 285A (D.K. *3658); 288B, on Picea log, 12 Aug. 2009, Karasiński 3738a (D.K.); 288D (D.K. *3738B); 313A, on Picea fallen trunk, 25 July 2009, Karasiński 3508 (D.K.); 289C (D.K. *3745B); 315A, on rotten Picea fallen trunk, 18 Aug. 2009, Karasiński 3889 (D.K.); 316B (D.K. *3788); 318D, on rotten stump of Picea, 13 Aug. 2009, Karasiński $3747 a$ (D.K.); 318D (D.K. *3869A); 319C (D.K. *3601); 319C, on Picea fallen log, 13 Aug. 2009, Karasiński 3754a (D.K.); 319C, on Picea fallen log, 13 Aug. 2009, Karasiński 3758 (D.K.); 340F, on rotten Picea fallen trunk, 6 July 2009, Karasiński 3296 (D.K.); 340G, on Picea fallen trunk, 16 Sept. 2009, Karasiński $4217 a$ (D.K.); 345A (D.K. *3715A); 346A (D.K. *3725); 370C (D.K. *3701); 370C, on rotten Picea fallen trunk, 10 Aug. 2009, Karasiński 3703c (D.K.); 373B, on Picea fallen trunk, 28 July 2009, Karasiński 3591 (D.K.); 374D, on Picea fallen trunk, 28 July 2009, Karasiński 3591 (D.K.); 375 B (D.K. *3892).

LiteRATURE REPORTS: Orłoś 1960 (as Leptoporus undosus), Domański et al. 1967 (as Tyromyces undosus), Szczepkowski et al. 2010 (as Oligoporus undosus), Gierczyk et al. 2013 (as Postia undosa), Niemelä 2013 (as Spongiporus undosus).

REMARKS. This species was described as a successor of Fomitopsis rosea [= Rhodofomes roseus (Alb. \& Schwein.) Kotl. \& Pouzar] (Spirin et al. 2006). The asterisked specimens grew with or on $F$. rosea dead basidiomata. The other specimens occurred without an evident connection with F. rosea. 


\section{Trametes gibbosa (Pers.) Fr.}

Fig. 20F

Specimens examined: 98B (D.K. 10985); 214D (Szczekotowo Reserve), on Carpinus fallen trunk, 17 Oct. 2014, Karasiński 11012 (D.K.); 334A, on Carpinus fallen trunk, 24 March 1993, Wotkowycki (H.M.W. M-1050); 372C, on Carpinus fallen trunk, 5 July 2009, Karasiński 3252H (D.K.); 399, on Carpinus fallen trunk, 31 Aug. 1973, Wojewoda s.n. (KRAM F-58164); BF, on Quercus trunk, Oct. 1955, Domański (KRAM F-SD 2704); BF, on deciduous trunk, 20 Sept. 1960, Domański (KRAM F-SD s.n., as Trametes gibbosa var. kalchbrenneri); BNP, Poprzeczny Tryb, on Carpinus fallen rather thin trunk, 14 Oct. 2008, Niemelä 8484 (KRA).

LITERATURE REPORTS: Błoński et al. 1888 (as T. gibbosa and T. Kalchbreneri), Błoński 1889a (as Daedalea gibbosa and D. kalchbrenneri), Siemaszko 1925, Pilát 1950, Orłóś 1951, 1960, 1961, Domański et al. 1967 (as Trametes gibbosa f. kalchbrenneri), Domański 1967, Anonymous 1968, Nespiak 1968, Domański et al. 1973 (as Trametes gibbosa f. kalchbrenneri), Bujakiewicz et al. 1992, Jaroszewicz 1996, Skirgiełło 1997, Szczepkowski et al. 2008, Bujakiewicz \& Kujawa 2010, Gierczyk et al. 2013, Niemelä 2013, Gierczyk et al. 2014.

\section{Trametes hirsuta (Wulfen) Pilát}

Specimens eXamined: 214D (D.K. 11000, 11011); 362A, on Carpinus fallen trunk, 24 March 1993, Wotkowycki (H.M.W. M-1053); 384D (H.M.W. M-1244); 385D (H.M.W. M-2736); 412B (H.M.W. M-0958); 429B (H.M.W. M-1052); 513B (H.M.W. M-1389); 517D (H.M.W. M-2855); 572C, on fallen trunk, 28 Aug. 1990, Wotkowycki (H.M.W. M-1051); BF, on Carpinus fallen branch, 8 Aug. 1962, Domański (KRAM F-SD 3677); BNP, Poprzeczny Tryb, Carpinus fallen tree, 14 Oct. 2008, Niemelä 8481 (KRA).

Literature RePORTS: Błoński et al. 1888 (as Polyporus hirsutus), Siemaszko 1923 (as Polyporus hirsutus), Karpiński 1949 (as Polystictus hirsutus), Pilát 1950, Nespiak 1956 (as Polystictus hirsutus), Orłoś 1960, 1961, Domański 1967, Nespiak 1968, Bujakiewicz et al. 1992, Bujakiewicz 1994, Jaroszewicz 1996, Skirgiełło 1997, Szczepkowski et al. 2008, Bujakiewicz \&Kujawa 2010, Gierczyk et al. 2013, Niemelä 2013, Gierczyk et al. 2014.

Trametes ochracea (Pers.) Gilb. \& Ryvarden

Fig. $20 \mathrm{G}$

Specimens examined: 214D (Szczekotowo Reserve), on Populus stump, 17 Oct. 2014, Karasiński
11019 (D.K.); 214D (D.K. 11015, 11027); 260D, on Populus log, 12 Oct. 2009, Karasiński 4625 (D.K.); 274B (H.M.W. M-1046); 306A (H.M.W. M-1045); 370D, on Populus log, 17 Oct. 2009, Karasiński 4800 (D.K.); 385B (H.M.W. M-1637); 413A (H.M.W. M-1215); 443 (W. Szafer Landscape Reserve), on Populus decorticate fallen trunk, 21 Apr. 2009, Karasiński 2841 (D.K.).

Literature RePORTs: Błoński et al. 1888 (as Polyporus zonatus), Karpiński 1949 (as Polystictus zonatus), Pilát 1950 (as Trametes zonata), Orłoś 1960 (as Trametes zonata), Domański et al. 1967 (as Trametes zonata), Domański 1967 (as Trametes zonata), Anonymous 1968 (as Trametes zonata), Bujakiewicz et al. 1992 (as Trametes multicolor), Skirgiełło 1997 (as Trametes multicolor), Gierczyk et al. 2013, Niemelä 2013, Gierczyk et al. 2014.

Trametes pubescens (Schumach.) Pilát

Fig. $20 \mathrm{H}$

SPeCimens EXAmined: 286B, on Carpinus fallen log, 19 Sept. 2011, Karasiński 6372 (D.K.); BF, on Carpinus branch, 18 Sept. 1960, Domański (KRAM F-SD 1022); BF, on deciduous trunk, 20 Sept. 1960, Domański (KRAM F-SD 949); BF, on deciduous branch, 24 Sept. 1965, Domański (KRAM F-SD 4891).

Literature REPORTS: Błoński 1889a (as Polyporus pubescens), Domański et al. 1967, Domański 1967, Nespiak 1968, Domański et al. 1973, Bujakiewicz et al. 1992, Skirgiełło 1997, 1998, Niemelä 2013.

\section{Trametes suaveolens (L.) Fr.}

Specimens eXamined: 374D, on trunk of Salix caprea living tree, 13 Oct. 2009, Karasiński 4647 (D.K.); 399, on trunk of Salix caprea, 21 Sept. 2011, Karasiński 6443 (D.K.).

LiterATURE REPORTS: Bujakiewicz et al. 1992, Skirgiełło 1997, Niemelä 2013.

\section{Trametes versicolor (L.) Pilát}

Fig. 21A

SPecimens examined: 182D, on Quercus fallen branch, 17 Oct. 2014, Karasiński 10996 (D.K.); 340A, on Fraxinus rotten stump, 22 Sept. 2010, Krasiński 5958 (D.K.); 412B (H.M.W. M-2832); 438C (H.M.W. M-1268); 439C (H.M.W. M-0962); 599A (H.M.W. M-1043).

Literature RePORTs: Błoński et al. 1888 (as Polyporus versicolor), Siemaszko 1925 (as Polyporus 
versicolor), Karpiński 1949 (as Polystictus versicolor), Orłoś 1951 (as Polystictus versicolor), Orłoś 1960, 1961, Domański et al. 1967, Domański 1967, Anonymous 1968, Nespiak 1968, 1970, Bujakiewicz et al. 1992, Jaroszewicz 1996, Skirgiełło 1997, Szczepkowski et al. 2008, Bujakiewicz \& Kujawa 2010, Gierczyk et al. 2013, Niemelä 2013, Gierczyk et al. 2014.

\section{Trametopsis cervina (Schwein.) Tomšovsky}

Fig. 21B

SPECIMENS EXAMINED: BF, on deciduous fallen trunk, 28 Aug. 1956, Domański (KRAM F-SD 489 as Trametes cervina); BF, on deciduous fallen trunk, 20 Oct. 1963, Domański (KRAM F-SD 3442 as Spongipellis delectans).

LiterATURE REPORTS: Domański 1965, 1967 (as Coriolellus cervinus), Domański et al. 1973 (as Trametes cervina), Niemelä 2013.

REMARKS. It seems to be a very rare species in the BF, not confirmed in recent fieldwork. Only two specimens are preserved in Domański's collection. Specimen KRAM F-SD 489 is fertile but in poor condition, and almost completely damaged by insects. The second specimen (KRAM F-SD 3442), misidentified by Domański as Spongipellis delectans, is sterile. It has a dimitic hyphal structure, which excludes Spongipellis. Based on a comparision with other specimens of Trametopsis cervina it was assigned to this species. Niemelä (2013) reported this species based on literature data (after Domański et al. 1973).

Trechispora candidissima (Schwein.) Bondartsev \& Singer

SPECIMENS EXAMINED: 370C, on rotten stump of Picea, 15 Oct. 2009, Karasiński 4719 (D.K.).

LiterATURE REPORTS: Domański 1967 (as Cristella candidissima), Niemelä 2013.

REMARKS. This is a rare species which differs from closely related species mostly by having small rod-like crystals covering subicular hyphae.
Trechispora hymenocystis (Berk. \& Broome) K. H. Larss. Fig. 21C

Specimens eXAmined: 500 (in the vicinity of Podolany Reserve), on bark of Picea fallen trunk, 27 Sept. 2006, Karasiński 1635 \& Piątek (KRAM F-47254); BNP, Poprzeczny Tryb, east of 'Mogiłki' graves, on Picea fallen thick trunk, 9 Sept. 2009, Niemelä 8573 (KRA).

LiterATURE REPORTS: Karasiński et al. 2009, Niemelä 2013.

REMARKS. This species differs from other poroid species of Trechispora P. Karst. mostly by having sphaerocysts on subicular hyphae.

Trechispora mollusca (Pers.) Liberta Fig. 21D

Specimens EXAmined: 315B, on very rotten wood, 18 Aug. 2009, Karasiński 3920 (D.K.); 373C, on Picea rotten stump, 13 July 2009, Karasiński 3467 (D.K.); 375B, on Pinus rotten log, 13 Oct. 2009, Karasiński 4656 (D.K.); 388C (W. Szafer Landscape Reserve), on very rotten wood, 19 Sept. 2007, Karasiński 0709197680 (D.K.); 387 (W. Szafer Landscape Reserve), on rotten wood, 20 Sept. 2013, Karasiński 9959 (D.K.).

LiTERATURE REPORTS: Domański 1972b (as Cristella mollusca), Niemelä 2013, Gierczyk et al. 2014.

Trichaptum abietinum (Dicks.) Ryvarden

Fig. 21E

SPeCimens eXAmined: 98B, on Picea fallen branch, 17 Oct. 2014, Karasiński 10987 (D.K.); 135D, on Picea trunk, 8 July 2009, Karasiński 3329A (D.K.); 285 (D.K. 5291B); 286D, on Picea fallen trunk, 14 Aug. 2009, Karasiński 3777A (D.K.); 369 (D.K. 4383B); 384C (H.M.W. M-1101); 412A (H.M.W. M-1324); 463A (H.M.W. M-1318); 464D (H.M.W. M-1100); BNP, Poprzeczny Tryb, Picea thick fallen trunk, 14 Oct. 2008, Niemelä 8474 (KRA).

LiteratURe REPORTS: Błoński et al. 1888 (as Polyporus abietinus), Błoński 1889a (as Polyporus abietinus), Siemaszko 1923 (as Polyporus abietinus), Pilát 1950 (as Trametes abietina), Orłoś 1960, 1961 (as Trametes abietina), Domański 1967, Anonymous 1968 (as Hirschioporus abietinus), Bujakiewicz et al.

Fig. 21. A - Trametes versicolor (L.) Pilát, B - Trametopsis cervina (Schwein.) Tomšovsky (photographed in Bieszczady National Park, SE Poland), C - Trechispora hymenocystis (Berk. \& Broome) K. H. Larss., D - Trechispora mollusca (Pers.) Liberta, E - Trichaptum abietinum (Dicks.) Ryvarden, F - Trichaptum biforme (Fr.) Ryvarden, G - Trichaptum fuscoviolaceum (Ehrenb.) Ryvarden, H - Tyromyces chioneus (Fr.) P. Karst. Photo D. Karasiński (A-H). 

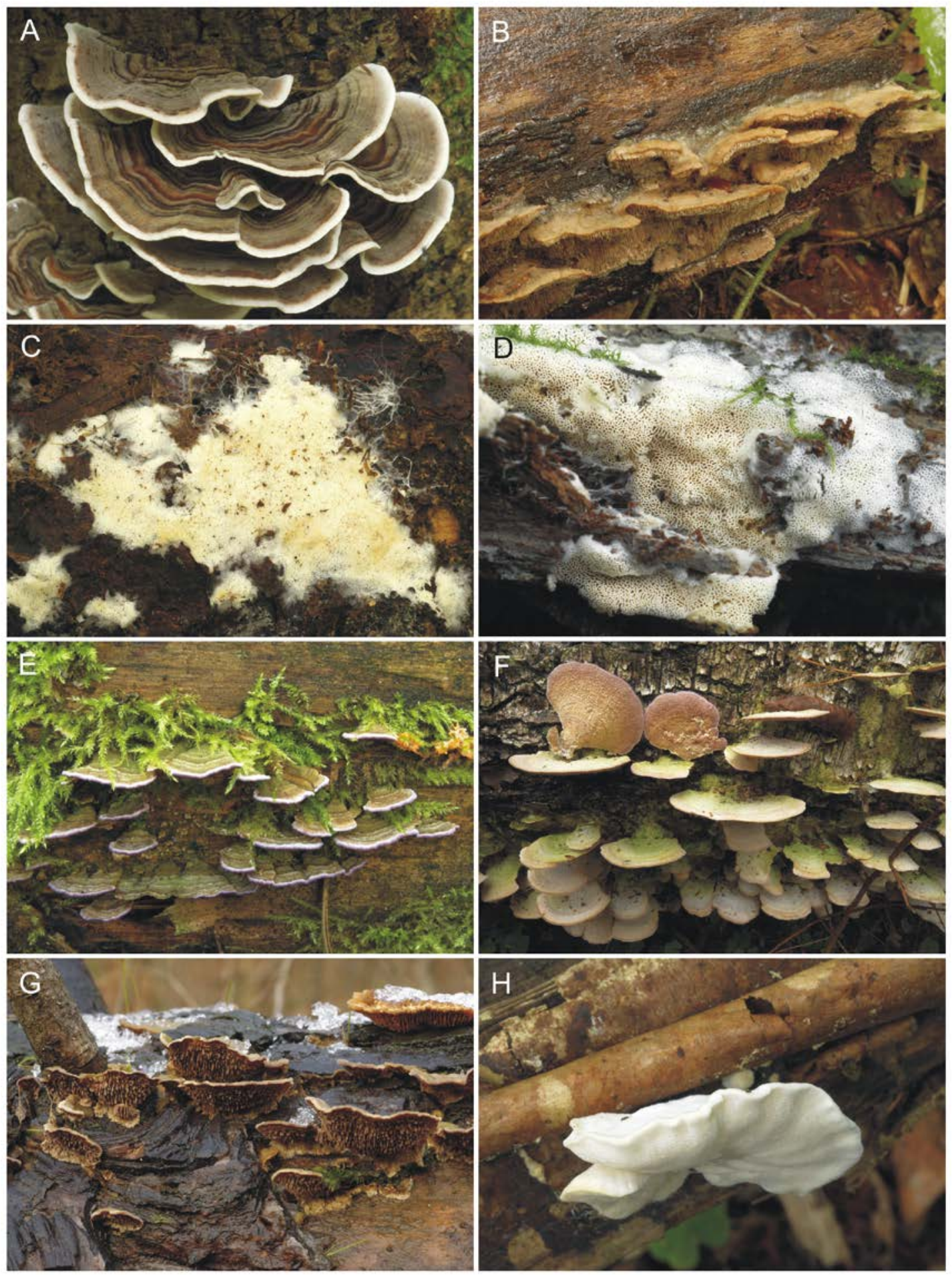
1992, Bujakiewicz 1994, Jaroszewicz 1996, Skirgiełło 1997, 1998, Szczepkowski et al. 2008, Bujakiewicz \& Kujawa 2010, Gierczyk et al. 2013, Niemelä 2013, Gierczyk et al. 2014.

\section{Trichaptum biforme (Fr.) Ryvarden Fig. 21F}

SPECIMENS EXAMINED: 402A, on Betula fallen trunk, 16 Oct. 2014, Karasiński 10938 (D.K.); 699C, on Betula pendula fallen trunk, 28 Sept. 2006, Wolkowycki (H.M.W. M-3611); BF, on Betula, Oct. 1956, Domański (KRAM F-SD 2660 as Hirschioporus pergamenus); BF, on hardwood, 22 May 1958, Domański (KRAM F-SD 2661 as Hirschioporus pergamenus); BF, on hardwood, Sept. 1956, Domański (KRAM F-SD 2388 as Hirschioporus pergamenus).

Literature REPORTS: Błoński 1889a (as Polyporus simulans), Pilát 1950 (as Trametes biformis), Orłoś 1960, 1961 (as Trametes biformis), Domański et al. 1967 (as Hirschioporus pergamenus), Domański 1967 (as Hirschioporus pergameus), Domański et al. 1973 (as Hirschioporus pergameus), Bujakiewicz et al. 1992, Wojewoda et al. 2002, Szczepkowski et al. 2008, 2011, Gierczyk et al. 2013, Niemelä 2013, Gierczyk et al. 2014.

\section{Trichaptum fuscoviolaceum (Ehrenb.)}

Ryvarden

Fig. $21 \mathrm{G}$

SPECIMENS EXAMINED: 385C, on Picea fallen trunk, 21 March 1991, Wolkowycki (H.M.W. M-1119); 437D (H.M.W. M-1330); 460C (H.M.W. M-1235); 600B (H.M.W. M-1104); BF, on Picea fallen trunk, 19 Sept. 1960, Domański (KRAM F-SD 883).

LITERATURE REPORTS: Błoński et al. 1888 (as Irpex fusco-violaceus), Błoński 1889a (as Sistotrema fuscoviolaceum), Siemaszko 1925, Bujakiewicz et al. 1992, Skirgiełło 1997, Niemelä 2013, Gierczyk et al. 2014 (as Trichaptum holli).

\section{Tyromyces chioneus (Fr.) P. Karst. Fig. $21 \mathrm{H}$}

Specimens examined: $\mathbf{3 4 0 F}$, on fallen branch of deciduous tree (Betula?), 16 Sept. 2009, Karasiński 4232 (D.K.); 340, on Carpinus fallen trunk, 22 Sept. 2009, Karasiński 4416 (D.K.); 340A, on dead standing Corylus avellana trunk, 22 Sept. 2010, Karasiński 5994 (D.K.); 437A (Sacharewo), on Corylus?, 9 Feb. 2004, Wołkowycki (H.M.W. M-3407); 730A (Starzyna reserve), on Betula pendula fallen branch, 23 Sept. 2004, Wołkowycki (H.M.W. M-3119).

LITERATURE REPORTS: Błoński 1889a (as Polyporus chioneus), Domański et al. 1967, 1973 (as Tyromyces albellus), Bujakiewicz et al. 1992, Skirgiełło 1997, Niemelä 2013.

\section{RECORDS WITH UNCERTAIN STATUS}

Albatrellus confluens (Fr.) Kotl. \& Pouzar

Literature RePORTS: Błoński et al. 1888 (as Polyporus politus), Domański et al. 1973 (cited specimen reported by Błoński et al. 1888).

REMARKs. Except for the first report, no additional specimens of the species have ever been published from the study area. This species was not confirmed by recent observations.

Amyloporia sordida (Ryvrden \& Gilb.) Vampola \& Pouzar

LITERATURE REPORTS: David \& Tortić 1984.

REMARKs. This species was not confirmed by recent observations. According to Vampola and Pouzar (1992) the specimen reported from the BNP by David and Tortić (1984) belongs to Amyloporia sitchensis.

Antrodia ramentacea (Berk. \& Broome) Donk

LiTERATURE REPORTS: Domański 1969a, Domański 1972b (as Coriolellus ramentaceus), Niemelä 2013.

REMARKs. The specimens cited by Domański (1969a, 1972b) were not found in KRAM F-SD. Niemelä (2013) reported this species from the BF based on literature data (after Domański 1969a). The occurence of this species in the BF has not been confirmed by recent observations.

Antrodia vaillantii (DC.) Ryvarden

Literature REPORTS: Błoński 1889a (as Polyporus Vaillantii).

REMARKS. Herbarium material is probably lost. The occurence of the species in the BF was not confirmed by recent studies.

Ceriporia reticulata (Hoffm.) Domański

LiTERATURE REPORTS: Błoński 1889a (as Polyporus farinellus), Domański 1965, 1967, 1972b, Niemelä 2013.

REMARKS. This species is quite common on 
dead hardwoods in Poland (Wojewoda 2003) but it seems to be very rare in the BF and was not confirmed by recent fieldwork. The specimens cited by Domański have not been found in KRAM F-SD. Niemelä (2013) reported this species based on literature data (after Domański 1967).

\section{Ceriporiopsis gilvescens (Bres.) Domański}

Literature REPORTS: Domański 1967, 1971, Niemelä 2013.

REMARKS. None of the several specimens of Ceriporiopsis glivescens stored in KRAM F-SD were collected in the study area. Niemelä (2013) reported this species from the BF based on literature data (after Domański 1971). The occurrence of this species in the BF was not confirmed during recent fieldwork.

Daedaleopsis tricolor (Bull.) Bondartsev \& Singer

LITERATURE REPORTS: Wojewoda 2002.

REMARKS. The specimen cited by Wojewoda (2002, KRAM F-SD 861) represents Daedaleopsis confragosa, with a small basidome and slightly reddened pileus surface.

\section{Dichomitus squalens (P. Karst.) Reid}

Literature REPORTS: Domański 1965 (as Coriolellus squalens), Domański 1972b, Niemelä 2013.

REMARKS. This species was not confirmed by recent observations. The only specimen in Domański's collection, labelled Coriolellus squalens collected in Białowieża (on pine planks on a railway bridge in Białowieża, 20 Oct. 1955, Domański, KRAM F-SD 273), does not belong to this species. The herbarium envelope contains a specimen having an effused-reflexed basidiome $c a 10 \times 4 \mathrm{~cm}$ in outline, with a widely effused resupinate part and an elongate, narrow pileus with a hirsute surface, circular pores 3-4 per mm with thick dissepiments, a trimitic hyphal structure with clamped generative hyphae 3-7 $\mu \mathrm{m}$, skeletal hyphae 4-6 $\mu \mathrm{m}$ wide, binding hyphae $2-4 \mu \mathrm{m}$ wide, basidia ca $20 \times 5 \mu \mathrm{m}$, and basidiospores 6-7 $\times 2-2.5 \mu \mathrm{m}$, cylindrical, slightly curved, hyaline, thin-walled and non-amyloid. This set of characters exclude Dichomitus squalens, especially due to the distinctly hirsute pileus, size and shape of basidiospores, and lack of dichotomously branched binding hyphae, but correct assignment to species is not possible. This indicates that the literature reports (Domański $1965,1972 b)$ are based on a misidentified specimen. Niemelä (2013) reported the species based on literature data (after Domański 1972b).

\section{Fuscoporia contigua (Pers.) G. Cunn.}

LITERATURE REPORTS: Błoński 1889a (as Ochroporus contiguus).

REMARKS. It was not found during recent fieldwork. The species seems to be very rare in the study area, or absent.

\section{Ganoderma adspersum (Schulzer) Donk}

Literature REPORTS: Domański et al. 1973, Niemelä 2013.

REMARKs. According to Domański et al. (1973) '(...) this fungus has been observed in Białowieża', but it was not confirmed during recent studies. Niemelä (2013) reported it from the BF based on literature data (after Domański et al. 1973).

Gloeophyllum trabeum (Pers.) Murrill

Literature RePORTS: Błoński et al. 1888 (as Polyporus trabeus).

REMARKS. Except for the literature report (Błoński et al. 1888) it was never published from the study area, and was not confirmed by recent observations.

\section{Oligoporus rennyi (Berk. \& Broome) Donk}

LITERATURE REPORTS: Domański 1972b (as Strangulidium rennyi), Niemelä 2013.

REMARKs. Herbarium material for the literature report (Domański 1972b) has not been found in KRAM F-SD, and the species was not found during recent fieldwork. Niemelä (2013) reported the species based on literature data (after Domański 1972b).

\section{Phellinus lundellii Niemelä}

LiTERATURE REPORTS: Domański et al. 1973, Niemelä 2013. 
REMARKS. It was not confirmed in recent studies. Herbarium material for the literature record from the BF (Domański et al. 1973) was not found among the specimens deposited in KRAM F-SD. Niemelä (2013) reported the species based on literature data (after Domański et al. 1973).

\section{Skeletocutis subincarnata (Peck) Jean Keller}

LITERATURE REPORTS: Domański 1972b (as Incrustoporia subincarnata).

REMARKS. It was not confirmed in recent fieldwork. The specimens preserved in KRAM F-SD under this name belong to Skeletocutis biguttulata (Piątek \& Cabała 2005).

\section{Conclusions}

The Białowieża Forest, and especially the area protected in Białowieża National Park, is a refuge for many endangered, rare and interesting organisms, especially wood-inhabiting fungi. Polypore species richness is very high there. Many relict fungal species of old-growth forest occur in the $\mathrm{BF}$ and have relatively large populations. The current knowledge of the poroid fungi in the Polish part of BF has been summarized here based on our examination of 1600 specimens and an analysis of about 100 published reports. In total, 210 species representing 80 genera are listed in this paper. Postia, Skeletocutis, Polyporus, Phellinus, Antrodia and Antrodiella are genera represented by the largest number of species. Fourteen species previously reported in the literature have uncertain status as polypores of the BF because they lack corresponding voucher specimens and were not confirmed in recent field studies. Another fourteen species are newly reported for the Białowieża Forest (mainly from Białowieża National Park), including 8 species reported for the first time from Poland: Antrodia hyalina, A. infirma, Antrodiella subradula, Junghuhnia fimbriatella, Postia folliculocystidiata, P. minusculoides, Skeletocutis chrysella and S. papyracea. Several very rare European polypores already reported from the Białowieża Forest in the $20^{\text {th }}$ century, such as Antrodia albobrunnea, Antrodiella foliaceodentata, Buglos- soporus pulvinus, Dichomitus albidofuscus and Gelatoporia subvermispora, were found at new localities, confirming their continuous occurrence in this forest. Antrodiella subradula, previously known from Asia, is reported for the first time from Europe.

The polypore diversity of the Polish part of the Białowieża Biosphere Reserve is unique, and much higher than in other European forests studied for their polypore biodiversity. A total of 394 poroid species are known from Europe (Ryvarden \& Melo 2014), and the half of them occur in the BF. About 235 poroid species have been noted from Poland so far; the vast majority of them occur in the BF, among them many species not reported from other areas of Poland. The only species not present in the BF are those strictly associated with mountain forests or mountain trees such as Abies alba and Fagus sylvatica, which are absent from the BF. As compared with the reports of polypore species from other Polish national parks, the BNP area seems to be a true polypore diversity hotspot. The Polish national park next-best investigated in terms of fungal diversity, Kampinos National Park (Kampinos Biosphere Reserve), hosts only 129 poroid species (Karasiński et al. 2015).

An analysis of the factors affecting such high diversity of poroid fungi in the $\mathrm{BF}$ is beyond the scope of this study. In general, however, we suggest that the polypore species richness of this area reflects the degree of naturalness of forest ecosystem processes there, with continuous availability of dead wood lying on the forest floor. Białowieża National Park in particular has a long history of continuity of forest growth in natural conditions. It was never extensively used by humans in the past. The absence of management over the centuries has ensured the continuous availability of a large amount of woody debris of different sizes and decay stages since the end of the last glaciation, serving as substrates for many relict, rare and endangered poroid fungi not found in most European forests.

ACKNOWLEDGEMENTS. We thank the authorities of Białowieża National Park (BNP) for granting permission to study fungi in the park, Piotr Mleczko (Kraków, 
curator of KRA) for loan of specimens, Grażyna Domian (Szczecin) for sharing a photograph of Spongipellis delectans, and the anonymous reviewer for helpful remarks and suggestions. This work is supported in part from statutory funds of the W. Szafer Institute of Botany, Polish Academy of Sciences, Kraków.

\section{REFERENCES}

AnONYmous 1968. Compte-rendu du IV-ème Congrès des Mycologues Européens. Warszawa 1966. Acta Mycol. 4: 181-196.

Benedix E. H. 1967. Im Pilzdorado von Bialowieza (Streiflichter vom IV. Europäischen Mykologenkongress 1966 in Warschau). Westfäl. Pilzbriefe. 6(7): 134-137.

Bernicchia A. 2005. Polyporaceae s.l. Fungi Europaei 10. Candusso, Alasio.

BŁoŃSKI F. 1889a. Spis roślin zarodnikowych zebranych lub zanotowanych w lecie w r. 1888 w puszczach: Białowieskiej, Świsłockiej i Ladzkiej. In: F. BŁoŃSKI \& K. DRYMMER, Sprawozdanie z wycieczki botanicznej, odbytej do Puszczy Białowieskiej, Ladzkiej i Świsłockiej w 1888 roku. Pamiętn. Fizjogr. 9: 63-115.

BŁońsKi F. 1889b. Fungi polonici novi. Hedwigia 28(4): 280-282.

BŁoński F., Drymmer K. \& EjSMOnd A. 1888. Sprawozdanie z wycieczki botanicznej odbytej do Puszczy Białowieskiej w lecie 1887 r. Pamiętn. Fizjogr. 8: 55-155.

Bujakiewicz A. 1994. Macrofungi in the alder forests of the Białowieża National Park. Mycol. Helv. 6(2): 57-76.

Bujakiewicz A 2002. New, rare and endangered fungi in the Białowieża Primeval Forest. Polish Bot. J. 47: 113-124.

Bujakiewicz A. 2003. The Białowieża Forest - refuge for endangered macrofungi. Parki Narodowe i Rezerwaty Przyrody 22(3): 323-346 (in Polish with English abstract).

Bujakiewicz A., Chlebicki A., Chmiel M., Cieśliński S., Czyżewska K., Faliński J. B., Glanc K., Glowacki Z., Klama H., Komorowska H., LisiewsKa M., MajewsKi T., MrozińsKa T., Mulenko W., Sadowska B., Skirgiello A., ZAŁUSKI T. \& ŻARNOWIEC J. 1992. Check-list of cryptogamous and seminal plant species recorded during the period 1987-1991 on the permanent plot V-100 (Project CRYPTO). In: J. B. FALIŃsKi \& W. MuŁenko (eds), Cryptogamous plants in the forest communities of Bialowieza National Park. Phytocoenosis 4 (N.S.), Archivum Geobotanicum 3: 1-48.

Bujakiewicz A. \& Kujawa A. 2010. Macrofungi in selected reserves of the Białowieża Forest. Parki Narodowe i Rezerwaty Przyrody 29(1): 3-26 (in Polish with English summary).
David A. \& Tortić M. 1984. Amyloporiella gen. nov. (Polyporaceae). Trans. Brit. Mycol. Soc. 83(4): 659-667.

DomańsKi S. 1959a. Certaines espèces du genre Poria de la forêt vierge de Białowieża en Pologne. Monogr. Bot. 8: 153-169 (in Polish with French summary).

DomaŃSKI S. 1959b. Deux rares porés: Leptoporus lapponicus (Rom.) Pil. et Phaeolus alboluteus (Ell. et Ev.) Pil. dans la forêt vierge de Białowieża en Pologne. Monogr. Bot. 8: 171-181 (in Polish with French summary).

Domański S. 1960. Leptoporus albidus (Schaeff. ex Secr.) Bourd. et Galz. and its diagnose. Acta Soc. Bot. Poloniae 29(4): 655-671 (in Polish with English summary).

DomańsKi S. 1962. Additamenta ad mycofloram lignicolam Reservati Publici ad Sanctam Crucem ("Góry Świętokrzyskie") (Polonia Centralis). Fragm. Florist. Geobot. 8(4): 509-517.

Domański S. 1963. Deux nouveaux genres des champignons de la groupe "Poria Pers. ex S. F. Gray". Acta Soc. Bot. Poloniae 32(4): 731-739 (in Polish with French summary).

Domański S. 1964a. Tyromyces Lowei (Pil. ex Pil.) Bond. en Pologne. Fragm. Florist. Geobot. 10(1): 81-88 (in Polish with French summary).

DoMAŃSKi S. 1964b. Wood-inhabiting fungi of Białowieża virgin forest in Poland I. Poria subacida (Peck) Sacc. and its diagnose. Acta Soc. Bot. Poloniae 33: 661-678 (in Polish with English summary).

Domański S. 1965. Flora Polska. Rośliny zarodnikowe Polski i ziem ościennych. Grzyby (Fungi). Podstawczaki (Basidiomycetes), Bezblaszkowe (Aphyllophorales), Żagwiowate I (Polyporaceae I), Szczecinkowate I (Mucronoporaceae I). Państwowe Wydawnictwo Naukowe, Warszawa.

DoMAŃSKI S. 1966. Wood-inhabiting fungi of Białowieża virgin forest in Poland IV. Poria albidofusca, sp. nov. and its diagnose. Acta Soc. Bot. Poloniae 35: 461-475 (in Polish with English summary).

DOMAŃSKI S. 1967. Specifity of wood-inhabiting mycoflora of the Białowieża National Park with a special regard to Aphyllophorales. Sylwan 111(1): 17-27 (in Polish with English summary).

DomaŃsKi S. 1969a. Wood-inhabiting fungi in Białowieża virgin forest in Poland VI. Antrodia rementacea (Berk. \& Br.) Donk. Acta Soc. Bot. Poloniae 38(1): 57-68 (in Polish with English summary).

DomaŃSKi S. 1969b. Wood-inhabiting fungi of Białowieża virgin forest in Poland VII. Schizopora paradoxa (Schrad. ex Fr.) Donk and its diagnose. Acta Soc. Bot. Poloniae 38(1): 69-81 (in Polish with English summary).

DoMAŃSKI S. 1969c. Wood-inhabiting fungi in Białowieża virgin forest in Poland VIII. Schizopora phellinoides (Pilát) comb. nov. and its diagnose. Acta Soc. Bot. Poloniae 38(2): 255-269 (in Polish with English summary). 
DoMAŃSKI S. 1969d. Wood-inhabiting fungi in Białowieża virgin forest in Poland X. Fibuloporia subvermispora (Pilát) Domań., comb. nov. and its diagnose. Acta Soc. Bot. Poloniae 38(3): 453-464 (in Polish with English summary).

DOMAŃSKI S. 1969e. Wood-inhabiting fungi in Białowieża virgin forest in Poland XI. Incrustoporia tschulymica (Pilát) Domań. and its diagnose. Acta Soc. Bot. Poloniae 38(3): 465-473 (in Polish with English summary).

DomaŃSKI S. 1970a. Wood-inhabiting fungi of Białowieża virgin forest in Poland XIII. Two species of Diplomitoporus Domań., gen. nov. Acta Soc. Bot. Poloniae 39(1): 191-207 (in Polish with English summary).

Domański S. 1970b. Wood-inhabiting fungi in Białowieża virgin forest in Poland XIV. Coriolus hoehnelii (Bres. in Höhn.) Bourd. \& Galz. Acta Soc. Bot. Poloniae 39(3): 521-530 (in Polish with English summary).

Domański S. 1970c. Wood-inhabiting fungi in Białowieża virgin forest in Poland XV. Polyporus dichrous Fr. and Polyporus pannocinctus Romell in culture. Acta Soc. Bot. Poloniae 39(3): 531-538 (in Polish with English summary).

Domański S. 1970d. Wood-inhabiting fungi in Białowieża virgin forest in Poland XVI. Coriolus foliaceo-dentatus (Nikol.) Domański, comb. nov. Acta Soc. Bot. Poloniae 39(4): 701-710 (in Polish with English summary).

Domański S. 1970e. Wood-inhabiting fungi of Białowieża virgin forest in Poland IX. Further studies on Ceriporiopsis placenta (Fr. sensu J. Erikss.) Domań. Acta Soc. Bot. Poloniae 39(1): 51-62 (in Polish with English summary).

DoMAŃsKi S. 1971. Wood-inhabiting fungi in Białowieża virgin forest in Poland XVII. Ceriporiopsis gilvescens (Bres.) Domański. Acta Soc. Bot. Poloniae 40: 295-303 (in Polish with English abstract and summary).

DomaŃSKI S. 1972a. Wood-inhabiting fungi of the Białowieża virgin forest in Poland XVIII. Amylocystis lapponica (Romell) Bond. \& Sing. Acta Soc. Bot. Poloniae 41(3): 425-431.

DomańSKI S. 1972b. Fungi, Polyporaceae I (resupinatae), Mucronoporaceae I (resupinatae). Foreign Scientific Publications Department of the National Center for Scientific, Technical and Economic Information, Warsaw.

Domański S. 1973. Poria elongata Overh. in Poland. Persoonia 7(2): 155-160.

DomańSKI S. 1984. Mala Flora grzybów. 1. Basidomycetes (Podstawczaki), Aphyllophorales (Bezblaszkowe). 4. Clavariaceae, Clavariadelphaceae, Clavulinaceae, Pterulaceae, Ramariaceae, Stephanosporaceae, Gomphaceae (II), Hericiaceae (II). Państwowe Wydawnictwo Naukowe, Warszawa - Kraków.

Domański S., OrŁoś H. \& SkirgieŁlo A. 1967. Flora Polska. Rośliny zarodnikowe Polski i ziem ościennych. Grzyby (Mycota). 3. Podstawczaki (Basidiomycetes), Bezblaszkowe (Aphyllophorales), Żagwiowate II (Polyporaceae pileatae), Szczecinkowate II (Mucronoporaceae pileatae), Lakownicowate (Ganodermataceae), Bondarcewowate (Bondarzewiaceae), Boletkowate (Boletopsidaceae), Ozorkowate (Fistulinaceae). Państwowe Wydawnictwo Naukowe, Warszawa-Kraków.

Domański S., OrŁoś H. \& Skirgiello A. 1973. Fungi. Polyporaceae II (pileate), Mucronoporaceae I (pileate), Ganodermataceae, Bondarzewiaceae, Boletopsidaceae, Fistulinaceae. Foreign Scientific Publications Department of the National Center for Scientific, Technical and Economic Information.

Domański Z. 2001. Grzyby Lasów Łochowskich. Authorpublisher, Warszawa.

Dominik T. 1963. Mycologische Notizen von 1945-60. Zesz. Nauk. Wyższej Szkoły Rolniczej w Szczecinie 10: 47-77 (in Polish with German summary).

Donk M. A. 1974. Check list of European polypores. Verh. Kon. Ned. Akad. Wetensch., Afd. Natuurk., Sect. 2 62: $1-469$.

FALIŃSKI J. B. 1986. Vegetation dynamics in temperate lowland primeval forest. Ecological studies in Białowieża Forest. Geobotany 8. Dr W. Junk Publishers, Dordrecht.

FALIŃSKi J. B. 2002. Basic information. In: J. B. FALIŃSKI (ed.), Białowieża Geobotanical Station of Warsaw University. Long-term studies. Bibliography. Data basis on vegetation and environment 1952-2002. Phytocoenosis 14 (N.S.), Supplementum Bibliographiae Geobotanicae 5: 9-16.

Gierczyk B., Kujawa A., Szczepkowski A. \& KarasińsKi D. 2014. Materials to the knowledge of mycobiota of the Białowieża Primeval Forest. Przegląd Przyrodniczy 25(1): 3-36 (in Polish with English abstract and summary).

Gierczyk B., Szczepkowski A. \& Kujawa A. 2013. The XVIII Fungi Exposition of the Białowieża Forest. Parki Narodowe i Rezerwaty Przyrody 32(2): 88-112 (in Polish with English abstract).

Große-Brauckmann H. \& Nuss I. 1991. Vier interessante Aphyllophorales-Arten aus dem Bayerischen Wald: Junghuhnia fimbriatella, Antrodiella citrinella (Poriaceae), Hypochnicium cymosum und Resinicium furfuraceum (Corticiaceae). Hoppea 50: 519-525.

Grzywacz A., Aleksandrowicz-TrzcińsKa M. \& SzCZePKOwSKI A. 1996. Phytopathological analysis of stands in Białowieża National Park and Białowieża Primeval Forest. In: P. PASChalis \& S. ZająCZKowsKi (eds), Biodiversity protection of Białowieża Primeval Forest. Selected papers, pp. 109-114. Fundacja Rozwój SGGW, Warszawa.

GumiŃsKa B. 1963. National Parks and Nature Reserves as territories of recent mycological investigations. Chrońmy Przyr. Ojczysta 19(3): 7-13 (in Polish with English summary).

Holec J. \& Pouzar Z. 1998. New records of rare fungi in the Šumava mountains (Czech Republic). II. Čas. Nár. Muz., Rada Přir. 167(1-4): 61-72. 
HoŁownIA I. 1974. New localities of some interesting species of fungi collected in the Northern Poland. Fragm. Florist. Geobot. 20: 535-542 (in Polish wit English summary).

Jaroszewicz B. 1993. Wystawa Grzybów Puszczy Białowieskiej. Parki Narodowe i Rezerwaty Przyrody 12(4): 95-96.

JARoszewicz B. 1996. Grzyby (Mycota) Puszczy Białowieskiej - gatunki zgromadzone na II Wystawie Grzybów Puszczy Białowieskiej. Parki Narodowe i Rezerwaty Przyrody 15(1): 47-53.

JęDrzejewsKa B. \& JęDRZEJEWSKi W. 1998. Predation in vertebrate communities. The Białowieża Primeval Forest as a case study. Springer, Berlin - Heidelberg - New York.

Karasiński D, Kujawa A., Gierczyk B., Ślusarczyk T., SzCZePKowski A. 2015. Macrofungi of the Kampinos National Park. Petit s.k. \& Kampinoski Park Narodowy, Izabelin (in Polish with English abstract).

Karasiński D., Kujawa A., Piątek M., Ronikier A. \& WolKOWYCKI M. 2009. Contribution to biodiversity assessment of european primeval forests: new records of rare fungi in the Białowieża Forest. Polish Bot. J. 54(1): 55-97.

Karasiński D., Kujawa A., Szczepkowski A. \& WolkoWYCKI M. 2010. Operat ochrony gatunków grzybów. In: B. Jaroszewicz (ed.), Plan ochrony Białowieskiego Parku Narodowego. Białowieski Park Narodowy, Białowieża.

KarpiŃsKi J. J. 1949. Materiały do bioekologii Puszczy Białowieskiej. Rozpr. Spraw. Inst. Badawczy Leśn., Ser. A, 56: $1-212$.

Kaufmann F. 1925. Die in Westpraußen gefunden Pilze aus der Famile: Polyporaceen Porlinge. Ber. Westpreuss. Bot.-Zool. Vereins Danzig 47: 1-23.

Kirk P. M., Cannon P. F., Minter D. W. \& Stalpers J. A. 2008. Ainsworth \& Bisby Dictionary of the Fungi. 10 Ed. CAB International, Wallingford.

KomorowsKa H. 1983. Włóknouszek dębowy Inonotus dryophilus (Berk) Murr. - rzadki grzyb zasługujący na ochronę. Wszechświat 84(7-8):166-167.

Kotkova V. M. \& Isaeva L. G. 2007. First record of Dichomitus albidofuscus (Polyporaceae, Basidiomycetes) in European part of Russia. Mikologia i Fitopatologia 41(5): 425-427 (in Russian with English summary).

Kotlaba F. \& LazebničeK J. 1967. The fourth European Mycological Congress, Poland 1966. Česká Mykol. 21(1): 54-59.

Kotlaba F. \& Pouzar Z. 1988. Type studies of polypores described by A. Pilát. I. Česká Mykol. 42(3): 129-136.

Kowalski T. \& ŁaKomy P. 1998. A new record of Heterobasidion annosum (Fr.) Bref. F group occurence in Poland in connection with interesting mycological findings. Phytopathol. Polon. 15: 49-55.

Kreisel H. (ed.) 1967. 4. Kongreß der europäischen Mykologen. Biol. Rundschau 5(2): 84-85.
KWIATKOWSKI W. 1994. Vegetation landscapes of Białowieża Forest. Phytocoenosis 6 (N.S.), Supplementum Cartographiae Geobotanicae 6: 35-87.

Łakomy P., Kowalski T. \& Werner A. 2000. Preliminary report on distribution of Heterobasidion annosum intersterility groups in Poland. Acta Mycol. 35(2): 303-309.

Malzahn E., Kwiatkowski W. \& Pierzgalski E. 2009. Przyroda nieożywiona. In: C. OKoŁów, M. Karaś \& A. Воєвот (eds), Białowieski Park Narodowy. Poznać, zrozumieć, zachować, pp. 18-36. Białowieski Park Narodowy, Białowieża.

Miettinen O., Niemelä T. \& Spirin W. 2006. Northern Antrodiella species: the identity of $A$. semisupina, and type studies of related taxa. Mycotaxon 96: 211-239.

Miettinen O., Spirin W. \& Niemelä T. 2012. Notes on the genus Aporpium (Auriculariales, Basidiomycota), with a new species from temperate Europe. Ann. Bot. Fenn. 49(5-6): 359-368.

NesPiaK A. 1956. Les champignons a chapeau dans les associations forestières de Białowieża (l'étude préliminaire). Fragm. Flor. Geobot. 2(2): 134-135 (in Polish with French summary).

NesPiaK A. 1959. The investigations on the character of the correlations between the higher fungi and wood associations in the National Park of Białowieża. Monogr. Bot. 8: 3-141 (in Polish with English summary).

NespiaK A. 1968. Grzyby (Fungi). In: J. B. Faliński (ed.), The National Park in Białowieża Primeval Forest, pp. 91-102. Państwowe Wydawnictwo Rolnicze i Leśne, Warszawa.

NesPiaK A. 1970. Grzyby i grzybobranie w Puszczy Białowieskiej. Białostockie Towarzystwo Kultury w Białymstoku, Białowieża.

Niemelä T. 1972. On Fennoscandian Polypores. 2. Phellinus laevigatus (Fr.) Bourd. \& Galz. and P. lundellii Niemelä, n. sp. Ann. Bot. Fenn. 9: 41-59.

Niemelä T. 1975. On Fennoscandian Polypores. 4. Phellinus igniarius, P. nigricans and P. populicola, n. sp. Ann. Bot. Fenn. 12: 93-122.

NiEMELÄ T. 1978a. The occurrence of some rare pore fungi in Finland. Ann. Bot. Fenn. 15: 1-6.

Niemelä T. 1978b. On Fennoscandian polypores 6. Antrodia plicata n.sp. Karstenia 18: 43-48.

Niemelä T. 1980. On Fennoscandian polypores 7. The genus Pycnoporellus. Karstenia 20: 1-15.

NiEmelä T. 1985. On Fennoscandian polypores 9. Gelatoporia n. gen. and Tyromyces canadensis, plus notes on Skeletocutis and Antrodia. Karstenia 25: 21-40.

Niemelä T. 2005. Polypores, lignicolous fungi. Norrlinia 13 : $1-320$.

Niemelä T. 2013. Polypores of the Białowieża Forest. Białowieski Park Narodowy, Białowieża. 
Niemelä T. \& DAI Y.-C. 1997. Polypore Skeletokutis lenis and its sib S. vulgaris. Ann. Bot. Fenn. 34: 133-140.

Niemelä T., Miettinen O. \& Manninen O. 2012. Aurantiporus priscus (Basidiomycota), a new polypore from old fallen conifer trees. Ann. Bot. Fenn. 49: 201-205.

Niemelä T., Wagner T., Fischer M. \& Dai Y. C. 2001. Phellopilus gen. nov. and its affinities within Phellinus s. lato and Inonotus s. lato (Basidiomycetes). Ann. Bot. Fenn. 38: $51-62$.

Oкоєów C. 2002. The value of Białowieża National Park for the development of biological sciences. Kosmos 51(4): 387-391 (in Polish with English summary).

OkoŁów. C. 2009. Historia ochrony. In: C. OKoŁów, M. Karaś \& A. Воєвот (eds), Białowieski Park Narodowy. Poznać, zrozumieć, zachować, pp. 10-16. Białowieski Park Narodowy, Białowieża.

OkoŁów C. 2012. Białowieża National Park (1921-2011) - an attempt at assessing the 90 years. Chrońmy Przyr. Ojczysta. 68(4): 288-301 (in Polish with English summary).

OrŁoś H. 1951. Przewodnik do oznaczania chorób drzew i zgnilizny drewna. Państwowe Wydawnictwo Rolnicze i Leśne, Warszawa.

Orıoś H. 1955a. Bohatství hub v Bělověžském pralese w Polsku. I. Mykol. Sborn. 32(1-2): 4-7.

Orıoś H. 1955b. Bohatství hub v Bělověžském pralese w Polsku. II. Mykol. Sborn. 32(4): 101-103.

Orıoś H. 1960. Badania nad funkcją ekologiczną grzybów $\mathrm{z}$ rodziny Polyporaceae $\mathrm{w}$ różnych typach lasu Białowieskiego Parku Narodowego. Prace Inst. Badawczego Leśn. 193: 5-100.

OrŁoś H. 1961. Badania ekologiczne nad mikoflorą niektórych typów lasu w Białowieskim Parku Narodowym. Prace Inst. Badawczego Leśn. 229: 57-106.

OrŁoś H. \& TwarowsKa I. 1967. Badania nad dynamiką zarodnikowania kilku gatunków grzybów z rodziny Polyporaceae. Prace Inst. Badawczego Leśn. 319: 203-226.

Parviainen J. 2005. Virgin and natural forests in the temperate zone of Europe. Forest Snow Landscape Res. 79(1/2): 9-18.

Parmasto E. 1968. Corticiaceae U.R.S.S. VI. Subfamily Botryohypochnoideae. Eesti N.S.V. Teaduste Akad. Toimet., Biol. 17: 404-410.

Pawlaczyk P. 2009. Zbiorowiska leśne. In: C. OkoŁów, M. Karaś \& A. BoŁbot (eds), Białowieski Park Narodowy. Poznać, zrozumieć, zachować, pp. 37-58. Białowieski Park Narodowy, Białowieża.

Peterken G. F. 1996. Natural woodland: ecology and conservation in northern temperate regions. Cambridge University Press, Cambridge.

PiąteK M. 2001. The genus Antrodiella (Fungi, Poriales) in Poland. Polish Bot. J. 46(2): 183-190.
Piątek M. 2002a. Antrodia malicola (Berk. \& M.A. Curtis) Donk. In: W. WoJEwoda (ed.), Atlas of the geographical distribution of fungi in Poland 2: 19-22. W. Szafer Institute of Botany, Polish Academy of Sciences, Kraków.

Piątek M. 2002b. Diplomitoporus lindbladii (Berk.) Gilb. \& Ryvarden. In: W. WoJEwodA (ed.), Atlas of the geographical distribution of fungi in Poland 2: 45-49. W. Szafer Institute of Botany, Polish Academy of Sciences, Kraków.

PiąteK M. 2002c. Pycnoporellus alboluteus (Ellis \& Everh.) Kotl. \& Pouzar. In: W. Wojewoda (ed.), Atlas of the geographical distribution of fungi in Poland 2: 85-87. W. Szafer Institute of Botany, Polish Academy of Sciences, Kraków.

Piątek M. 2002d. Pycnoporellus fulgens (Fr.) Donk. In: W. Wojewoda (ed.), Atlas of the geographical distribution of fungi in Poland 2: 89-94. W. Szafer Institute of Botany, Polish Academy of Sciences, Kraków.

Piątek M. 2003. Notes on Polish polypores. 3. Four rare species of old-growth forests. Polish Bot. J. 48(2): 131-144.

PiąTeK M. 2005a. Amylocystis lapponica (Romell) Singer. In: W. Wojewoda (ed.), Atlas of the geographical distribution of fungi in Poland 3: 5-8. W. Szafer Institute of Botany, Polish Academy of Sciences, Kraków.

PiąteK M. 2005b. Antrodia albobrunnea (Romell) Ryvarden. In: W. WoJewoda (ed.), Atlas of the geographical distribution of fungi in Poland 3: 9-11. W. Szafer Institute of Botany, Polish Academy of Sciences, Kraków.

PiąteK M. 2005c. Skeletocutis odora (Sacc.) Ginns. In: W. WoJEWODA (ed.), Atlas of the geographical distribution of fungi in Poland 3: 103-106. W. Szafer Institute of Botany, Polish Academy of Sciences, Kraków.

PiąteK M. \& Cabala J. 2005. New and noteworthy polypores from Poland with validation of the family Phaeotrametaceae. Mycotaxon 91: 173-183.

Piątek M., Seta D. \& Szczepkowski A. 2004. Notes on Polish polypores 5. Synopsis of the genus Spongipellis. Acta Mycol. 39(1): 25-32.

Piątek M. \& Wolkowycki M. 2005. Hapalopilus croceus (Pers.: Fr.) Bondartsev \& Singer. In: W. WoJEwoda (ed.), Atlas of the geographical distribution of fungi in Poland 3: 35-38. W. Szafer Institute of Botany, Polish Academy of Sciences, Kraków.

Pieri M. \& Rivorre B. 2006. A propos du complexe Postia sericeomollis. Bull. Mens. Soc. Linn. Lyon 75(3): 113-133.

Pilát A. 1950. Contribution to the knowledge of the Hymenomycetes of Białowieża virgin forest in Poland. Stud. Bot. Čechoslov. 11: 145-173.

RenVall P. 1992. Basidiomycetes at the timberline in Lapland 4. Postia lateritia n. sp. and its rust coloured relatives. Karstenia 32: 43-60. 
Renvall P. 1993. Antrodia infirma, an addition to the Swedish polypore flora. Windahlia 20: 35-37.

Renvall P. \& Niemelä T. 1992. Basidiomycetes at the timberline in Lapland 3. Two new boreal polypores with intricate hyphal system. Karstenia 32: 29-42.

Ryvarden L. \& Gilbertson R. L. 1993. European polypores. 1. Synopsis Fungorum 6. Fungiflora, Oslo.

RyVARDEN L. \& GILbERTSON R. L. 1994. European polypores. 2. Synopsis Fungorum 7. Fungiflora, Oslo.

Ryvarden L. \& Melo I. 2014. Poroid fungi of Europe. Synopsis Fungorum 31. Fungiflora, Oslo.

SiemaszKo W. 1923. Fungi bialowiezenses exsiccati. Centuria prima. Acta Insitituti Phytopathologici Scholae Superioris Agroculturae Varsaviensis 2: 1-27.

Siemaszko W. 1925. Fungi bialowiezenses exsiccati. Centuria secunda. Acta Insitituti Phytopathologici Scholae Superioris Agroculturae Varsaviensis (1925): 1-17.

SKIRGIEŁŁo A. 1965. Matériaux à la connaissance de la distribution géographique des champignons supérieurs en Europe. I. Acta Mycol. 1: 23-26 (in Polish with French summary).

SkirgieŁlo A. 1970. Matériaux à la connaissance de la distribution géographique des champignons supérieurs en Europe. III. Acta Mycol. 6: 101-123 (in Polish with French summary).

SkirgieŁlo A. 1972. Matériaux à la connaissance de la distribution géographique des champignons supérieurs en Europe. IV. Acta Mycol. 8(2): 191-218 (in Polish with French summary).

Skirgielıo A. 1976. Matériaux à la connaissance de la distribution géographique des champignons supérieurs en Europe. V. Acta Mycol. 12(2): 155-189 (in Polish with French summary).

SkIRgieŁlo A. 1984. Matériaux à la connaissance de la distribution géographique des champignons supérieurs en Europe. VI. Acta Mycol. 20(1): 129-157 (in Polish with French summary).

SkIRgIEŁlo A. 1997. Aphyllophorales. In: J. B. FALIŃSKI \& W. MuŁenko (eds), Cryptogamous plants in the forest communities of Białowieża National Park. Ecological Atlas (Project CRYPTO 4). Phytocoenosis 9 (N.S.), Supplementum Cartographiae Geobotanicae 7: 275-300.

SkIRGIEŁlo A. 1998. Macromycetes of oak-hornbeam forests in the Białowieża National Park - monitoring studies. Acta Mycol. 33: 171-189.

SoKóŁ S. 2000. The Ganodermataceae of Poland. Taxonomy, ecology and distribution. Prace Naukowe Uniwersytetu Ślaskiego w Katowicach 1867: 1-134 (in Polish with English and German summary).

Spirin W., Miettinen O., Pennanen J., Kotiranta H. \& NieMELÄ T. 2013a. Antrodia hyalina, a new polypore from Russia, and A. leucaena, new to Europe. Mycol. Progr. 12(1): 53-61.
Spirin V., Vlasák J., Niemelä T. \& Miettinen O. 2013b. What is Antrodia sensu stricto? Mycologia 105(6): 15551576.

Spirin W. A., Zmitrovich I. V., WASSER S. P. 2006. Oligoporus balsameus - rare Eurasian species, plus notes on related taxa. Mycotaxon 97: 73-82.

SzCZEPKA M. 1989. The fungus Fomitopsis rosea, an inhabitant of primeval coniferous forests. Chrońmy Przyr. Ojczysta 45(4): 22-39 (in Polish with English summary).

Szczepka M. \& SokóŁ S. 1991. Grifola frondosa (Dicks.: Fr.) S.F. Gray in Poland. Acta Biol. Siles. 19: 103-119 (in Polish with English abstract).

Szczepkowski A., Kujawa A., Karasiński D. \& GierczyK B. 2008. Fungi gathered for the XIV Fungi Exposition of the Białowieża Forest. Parki Narodowe i Rezerwaty Przyrody 27(4): 115-133 (in Polish with English summary).

Szczepkowski A., Kujawa A., Karasiński D. \& GierczyK B. 2011. The XVII Fungi Exposition of the Białowieża Forest in Hajnówka. Parki Narodowe i Rezerwaty Przyrody 30(3-4): 129-134 (in Polish with English abstract).

Szczepkowski A., Kujawa A., Karasiński D. \& Konik J. 2010. The XVI Fungi Exposition of the Białowieża Forest - general characteristic of gathered fungi. Parki $\mathrm{Na}$ rodowe i Rezerwaty Przyrody 29(4): 116-120 (in Polish with English abstract).

Szczepkowski A. \& PiętKa J. 2008. Nature reserves as refuge of Grifola frondosa (Dicks.: Fr.) Gray in central Poland. Parki Narodowe i Rezerwaty Przyrody 27(4): 3-13 (in Polish with English abstract).

ToMšovSKÝ M. 2012. Delimination of an almost forgotten species Spongipellis litschaueri (Polyporales, Basidiomycota) and its taxonomic position within the genus. Mycol. Progr. 11(2): 415-424.

Vampola P. \& Pouzar Z. 1992. Contribution to the knowledge of a rare resupinate polypore Amyloporia sitchensis. Česka Mykol. 46(3-4): 213-222.

Vampola P. \& Vlasák J. 1992. Dichomitus albidofuscus and Gelatoporia subvermispora - two new polypores for Czechoslovakia. Česka Mykol. 46(1-2): 114-120.

Vampola P., Ordynets A. \& Vlasák J. 2014. The identity of Postia lowei (Basidiomycota, Polyporales) and notes on related or similar species. Czech Mycol. 66(1): 39-52.

VlasÁK J., Kout J. \& DvořaK D. 2010. Taxonomical position of polypore Dichomitus albidofuscus: Donkiporia albidofusca comb. nov. Mycol. Progr. 9: 147-150.

Wojewoda W. 1977. Flora Polska. Rośliny zarodnikowe Polski i ziem ościennych. Grzyby (Mycota) 8. Podstawczaki (Basidiomycetes), Trzęsakowe (Tremellales), Uszakowe (Auriculariales), Czerwcogrzybowe (Septobasidiales). Państwowe Wydawnictwo Naukowe, Warszawa-Kraków. 
WoJEWODA W. 1979. The geographical distribution of the tremellaceous fungi in Poland. Acta Mycol. 15(1): 75-144 (in Polish with English summary).

Wojewoda W. 2002. Daedaleopsis tricolor (Bull.: Fr.) Bondartsev \& Singer. In: W. WoJEwoda (ed.), Atlas of the geographical distribution of fungi in Poland 2: 39-44. W. Szafer Institute of Botany, Polish Academy of Sciences, Kraków.

Wojewoda W. 2003. Checklist of Polish larger Basidiomycetes. Biodiversity of Poland 7. W. Szafer Institute of Botany, Polish Academy of Sciences, Kraków.
Wojewoda W., Heinrich Z. \& Komorowska H. 2002. Trichaptum biforme (Fr.) Ryvarden. In: W. WoJEWODA (ed.), Atlas of the geographical distribution of fungi in Poland 2: 119-126. W. Szafer Institute of Botany, Polish Academy of Sciences, Kraków.

Zhao Ch.-L, Cui B.-K. \& Steffen K. T. 2013. Yuchengia, a new polypore ganus segregated from Perenniporia (Polyporales) based on morphological and molecular evidence. Nordic J. Bot. 31(3): 331-338.

Received 8 September 2015 